\title{
INORGANIC ANALYSES OF VOLATILIZED AND CONDENSED SPECIES WITHIN PROTOTYPIC DEFENSE WASTE PROCESSING FACILITY (DWPF) CANISTERED WASTE (U)
}

by

\section{M. Jantzen}

Westinghouse Savannah River Company

Savannah River Site

Aiken, South Carolina 29808

This report was prepared in connection with work done under Contract No. DE-AC09-89SR18035 with the U.S. Department of Energy. By acceptance of this report, the publisher and/or recipient acknowledges the U.S. Government's right to retain a nonexclusive, royalty-free license in and to any copyright covering this report, along with the right to reproduce and to authorize others to reproduce all or part of the copyrighted report.

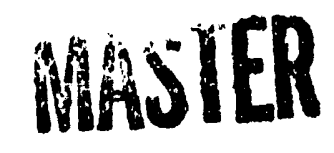




\section{DISCLAIMER}

This report was prepared as an account of work sponsored by an agency of the United States Government. Neither the United States Government nor any agency thereof, nor any of their employees, makes any warranty, express or implied, or assumies any legal liability or responsibility for the accuracy, completeness, or usefulness of any information, apparatus, product, or process disclosed, or represents that its use would not infringe privately owned rights. Reference herein to any specific commercial product, process, or service by trade name, trademark, manufacturer, or otherwise does not necessarily constitute or imply its endorsement, recommendation, or favoring by the United States Government or any agency thereof. The views and opinions of authors expressed herein do not necessarily state or reflect those of the United States Government or any agency thereof.

This report has been reproduced directly from the best availabie copy.

Available to DOE and DOE contractors from the Office of Scientific and Technical Information, P.O. Box 62, Oak Ridge, TN 37831; prices available from (615) 576-8401, FTS 626-8401.

Available to the public from the National Technical Information Service, U.S. Department of Commerce, 5285 Port Royal Rd., Springfield, VA 22161. 
WSRC-TR-92-260, Rev. 0

Keywords: glass, inorganic species, canisters, DWPF, canistered waste forms,

volatilization, condensation

\section{INORGANIC ANALYSES OF VOLATILIZED AND CONDENSED SPECIES WITHIN PROTOTYPIC DEFENSE WASTE PROCESSING FACILITY (DWPF) CANISTERED WASTE (U)}

\section{CAROL M. JANTZEN}

Approved by

E.W. Holtzscheiter, Research Manager Defense Waste Processing Technology

Publication Date: June 30, 1992

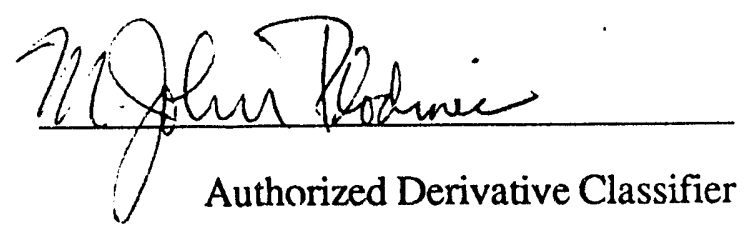

Westinghouse Savannah River Co. Savannah River Site Aiken, SC 29808

PREPARED FOR THE U.S. DEPARTMENT OF ENERGY UNDER CONTRACT DE-AC09-89SR18035 
This page intentionally left blank.

- 2 - 


\section{ABSTRACT}

The high-level radioactive waste currently stored in carbon steel tanks at the Savannah River Site (SRS) will be immobilized in a borosilicate glass in the Defense Waste Processing Facility (DWPF). The canistered waste will be sent to a geologic repository for final disposal. The Department of Energy has defined requirements which the canistered waste must meet to be acceptable for disposal in the repository. These requirements are the Waste Acceptance Preliminary Specifications (WAPS) which require the identification of any inorganic phases that may be present in the canister that may (1) lead to internal corrosion of the canister or (2) that could potentially adversely affect normal canister handling during storage and/or during an accident.

Borosilicate waste glass is poured into the canister at a temperature of about $1000{ }^{\circ} \mathrm{C}$ and the canister is filled under a slight vacuum. Potentially volatile halide and sulfate species $(\mathrm{NaCl}, \mathrm{NaF}$, $\mathrm{NaI}, \mathrm{CaF}_{2}, \mathrm{Na}_{2} \mathrm{SO}_{4}, \mathrm{CaSO}_{4}, \mathrm{BaSO}_{4}$ ) are present in the waste but concentrations are limited by process constraints. During vitrification, volatilization of mixed $(\mathrm{Na}, \mathrm{K}, \mathrm{Cs}) \mathrm{Cl},(\mathrm{Na}, \mathrm{K}, \mathrm{Cs})_{2} \mathrm{SO}_{4}$, $(\mathrm{Na}, \mathrm{K}, \mathrm{Cs}) \mathrm{BF}_{4},(\mathrm{Na}, \mathrm{K})_{2} \mathrm{~B}_{4} \mathrm{O}$ and $(\mathrm{Na}, \mathrm{K}) \mathrm{CrO}_{4}$ species from the surface of the glass melt have been observed to condense in the melter off-gas line above the glass melt pool and in the cyclone separator which is installed on the canister pour spout vacuum line. The volatilization and vapor phase condensation of alkali halides and sulfates throughout the DWPF melter system, including the pour spout vacuum line above the canister, indicated that similar species could possibly condense on the interior of the canister.

A full-scale DWPF prototypic canister that had been filled during Campaign 10 of the SRS Scale Glass Melter was sectioned and examined. Mixed $(\mathrm{Na}, \mathrm{K}) \mathrm{Cl},(\mathrm{Na}, \mathrm{K})_{2} \mathrm{SO}_{4}$, $(\mathrm{Na}, \mathrm{K})$ borates, and a $(\mathrm{Na}, \mathrm{K})$ fluoride phase (either $\mathrm{NaF}$ or $\mathrm{Na}_{2} \mathrm{BF}_{4}$ ) were identified as inorganic species on the interior canister walls, neck, and shoulder above the melt pour surface. Similar deposits were found on the glass melt surface and on glass fracture surfaces. Chromates were not identified on the interior of the canister. Spinel crystals were found associated with the glass pour surface. Vaporization of species from the melt as it enters the canister and condensation on the interior canister shoulder and neck as the glass is poured is the most likely mechanism by which the halide and sulfate species are deposited. Reference amounts of the halides and sulfates were found retained in the glass and the glass chemistry, including the distribution of the halides and sulfates, was homogeneous.

Rust was visibly present and identified in the DWPF prototypic canister. In all cases where rust was observed, heavy metals $(\mathrm{Zn}, \mathrm{Ti}, \mathrm{Sn})$ from the cutting blade/fluid were present indicating that the rust was a reaction product of the cutting fluid with the glass and with the heat sensitized canister or with carbon steel contamination on the canister interior which occurs during fabrication and handling. Measured relative humidity in sealed simulated waste glass canisters indicates that only minimal water vapor is present so that internal corrosion of the canister, as observed in this sectioned canister, will not occur. 
This page intentionally left blank.

$-4-$ 
Abstract

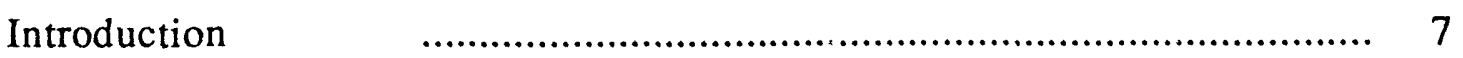

Experimental

Glass and Deposit Sampling ................................................... 9

Glass Composition Analyses ……………….......................... 9

X-ray Diffraction and Scanning Electron Microscopy Analysis ................ 12

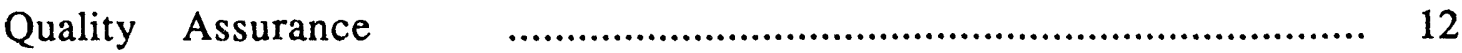

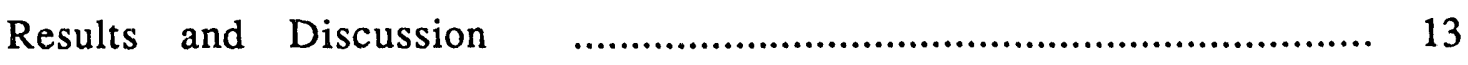

Glass Composition Analyses …................................................ 13

Deposit and Reaction Product Analyses ....................................... 13

Conclusions

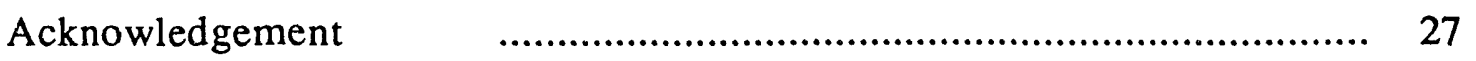

References

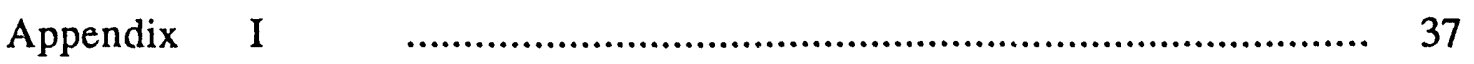


This page intentionally left blank.

$-6-$ 


\section{INORGANIC ANALYSES OF VOLATILIZED AND CONDENSED SPECIES WITHIN PROTOTYPIC DEFENSE WASTE PROCEŚSING FACILITY (DWPF) CANISTERED WASTE (U)}

\section{INTRODUCTION}

The high-level radioactive waste currently stored in carbon steel tanks at the Savannah River Site (SRS) will be immobilized in a borosilicate glass in the Defense Waste Processing Facility (DWPF). The canistered waste will be sent to a geologic repository for final disposal. The Department of Energy has defined requirements which the canistered waste must meet to be acceptable for disposal in the repository. These requirements are the Waste Acceptance

Preliminary Specifications (WAPS). ${ }^{1}$ Specification 3.9 of the WAPS is the chemical compatibility specification. Specification 3.9 requires the identification of any inorganic phases that may be present in the canister that may (1) lead to internal corrosion of the canister or (2) that could potentially adversely affect normal canister handling during storage and/or during an accident. Internal canister corrosion, chemical species, and any reaction products forming due to chemical interactions within the canistered waste after exposure to temperatures up to the glass transition temperature are to be identified.

The vitrification process operates at $1150^{\circ} \mathrm{C}$ at a nominal melter residence time of 65 hours. ${ }^{2}$ The borosilicate waste glass is poured into a canister at a temperature of about $1000{ }^{\circ} \mathrm{C}$ under a slight vacuum. Potentially volatile halide and sulfate species ( $\mathrm{NaCl}, \mathrm{NaF}, \mathrm{NaI}, \mathrm{CaF}_{2}, \mathrm{Na}_{2} \mathrm{SO}_{4}, \mathrm{CaSO}_{4}$, $\mathrm{BaSO}_{4}$ ) are present in the waste. ${ }^{3-5}$ Process constraints limit the total amount of $\mathrm{NaCl}$ to $<0.6$ $\mathrm{wt} \%, \mathrm{NaF}$ to $<0.1 \mathrm{wt} \%$, and $\mathrm{SO}_{4}{ }^{2-}$ species to $<0.4 \mathrm{wt} \%$ of the glass. ${ }^{6}$ The seven projected DWPF glass compositions described in the DWPF Waste Form Compliance Plan (WCP) $2,4-5$ have $\mathrm{NaCl}$ concentrations of 0.09 to $0.31 \mathrm{wt} \%, \mathrm{NaF}$ concentration $\mathrm{s}$ of 0.04 to $0.08 \mathrm{wt} \%$, NaI concentrations of $<0.0009 \mathrm{wt} \%, \mathrm{CaF}_{2}$ concentrations of $<0.008 \mathrm{wt} \%$, and $\mathrm{SO}_{4}{ }^{2-}$ concentrations (from $\mathrm{Na}_{2} \mathrm{SO}_{4}+\mathrm{CaSO}_{4}+\mathrm{BaSO}_{4}$ ) of 0.165 to $0.284 \mathrm{wt} \%$. These waste species are melted with borosilicate glass frit chemicals and with other alkali borate waste stream species to form the borosilicate nuclear waste glass.

During vitrification, the species present in the waste, frit, and alkali borate waste stream are homogenized, and volatilization of chemical constituents with the highest vapor pressures occurs. Volatilization of mixed ( $\mathrm{Na}, \mathrm{K}, \mathrm{Cs}) \mathrm{Cl},\left(\mathrm{Na}, \mathrm{K}, \mathrm{Cs}_{2} \mathrm{SO}_{4},(\mathrm{Na}, \mathrm{K}, \mathrm{Cs}) \mathrm{BF}_{4},(\mathrm{Na}, \mathrm{K})_{2} \mathrm{~B}_{4} \mathrm{O}_{7}\right.$ and $(\mathrm{Na}, \mathrm{K}) \mathrm{CrO}_{4}$ species from the surface of the glass melt have been observed to condense in the melter off-gas line above the glass melt pool. 7-9 In addition, deposits highly enriched in $\mathrm{NaCl}$, $\mathrm{Na}_{2} \mathrm{SO}_{4}$ and $\mathrm{KBF}_{4}$ have been observed in the cyclone separator which is installed on the canister

pour spout vacuum line. ${ }^{7}$ The volatilization and vapor phase condensation of alkali halides and sulfates throughout the DWPF melter system, including the pour spout vacuum line above the canister, indicated that similar species could possibly condense on the interior of the canister. 
The presence of halides and fluorides on the canister interior is not of concern unless water vapor is present. Halides in the presence of water vapor could initiate internal stress corrosion cracking of the $304 \mathrm{~L}$ canister. Since the glass enters the canister at $\sim 1000^{\circ} \mathrm{C}$, and the canister is under a slight vacuum, free liquids will not enter the canister with the molten glass stream. Any liquids or moisture present in the canister prior to pouring are unlikely to remain due to the heat of the molten glass and the reduced pressure. ${ }^{2}$ The only path by which water or water vapor could enter the canister is during the decontamination of the canister with a frit/water slurry. However, a shrinkfit seal has been developed to ensure that this slurry does not enter the canister. ${ }^{10}$ This temporary canister closure seal will be inserted into the canister neck after filling, and before canister decontamination, to prevent inleakage of the decontamination slurry. Every temporary canister closure will be tested to ensure that it is water tight.

The measured relative humidity in sealed simulated waste glass canisters ranged from 5.5 to $18.5 \%$, consistent with the absence of water which would increase the relative humidity to $100 \% .11$ The measured relative humidity in the waste glass canisters agrees with previously calculated values. ${ }^{12-13}$ The only difference in the gas phases noted within the canister was a decreased $\mathrm{CO}_{2}$ content relative to air. No other organics, pyrophorics, combustibles, or explosives have been identified in the gas phase of the canistered waste. ${ }^{11}$

The present study was initiated to document the inorganic (non-waste glass) species, e.g. the waste glass volatiles, that might have condensed on the interior of the stainless steel waste canisters and any reaction products observed. The mechanism by which the reaction products formed was also to be determined. 


\section{EXPERIMENTAL}

\section{Glass and Deposit Sampling}

A full-scale DWPF prototypic canister $(\sim 120$ " height $)$ that had been filled during the SRS Scale Glass Melter (SGM) Campaign 10 was sectioned at 80" height with a band saw (Figure 1). The second canister of the SGM 10 Campaign, SGM 10-2 (Canister A-8), was chosen for sampling because the SGM 10 feed contained the reference amounts of halides and sulfates. ${ }^{14}$ Sectioning of the canister occurred about 6 months prior to sampling.

DWPF canisters are filled to a reference height of $\sim 91$ " with glass. Sectioning canister $10-2$ from 80 to 120 " provided about 11 " of glass for sampling (Figure 1). The glass was chiseled out of the canister in fist size pieces in order to ensure that the orientation of the melt surface was preserved for sampling of deposits. Glass samples were taken at the 80 " sectioned surface near the edge and in the center of the canister (Figure 2) for determination of the glass composition. Samples of glass from the edge and center of the canister were also taken at 86 " and 89 " heights (Figure 2) to look for chemical variation of the glass and/or halide and sulfate content in the canister.

Forty four glass samples, including the melt surface, fracture surfaces, and glass adjacent to the canister walls were sampled for solids/deposit analysis. When the glass was completely removed from the canister, visible deposits on the shoulder and neck of the canister (Figure 3) were removed with scctch tape for solids/deposit analysis. Two additional samples of deposits were collected by flushing off the canister shoulder with deionized water. An eyedropper was used to transfer the liquid to a watch glass. One half of each liquid sample was dried at $900^{\circ} \mathrm{C}$ for solids analysis and one half sent for chemical analysis.

\section{Glass Composition Analyses}

The six glass samples were analyzed in duplicate by the following techniques:

- Dissolution by $\mathrm{Na}_{2} \mathrm{O}_{2}$ with an $\mathrm{HCl}$ uptake

- ICP for Al, Ca, Fe, Mg, Mn, Li, Si, Cr, B, Sr, Ti, P, Ba, Pb, Mo, Zn, Cu, Ni, $\mathrm{La}, \mathrm{Cd}$

- Dissolution by $\mathrm{HCl} / \mathrm{IIF}$ microwave

- ICP for $\mathrm{Na}, \mathrm{Zr}, \mathrm{Sn}$

- AA for $\mathrm{Na}, \mathrm{K}, \mathrm{Si}$

- Dissolution by $\mathrm{Na}_{2} \mathrm{O}_{2}$ with a $\mathrm{H}_{2} \mathrm{O}$ uptake

- IC for $\mathrm{SO}_{4}, \mathrm{NO}_{3}$, and $\mathrm{PO}_{4}$

- ISE for $\mathrm{Cl}$ and $\mathrm{F}$

where ICP is Inductively Coupled Plasma Spectroscopy, AA is Atomic Absorption analysis, IC is Ion Chromatography analysis, and ISE is Ion Selective Electrode analysis. The liquid water flush deposits were submitted for $\mathrm{Cl}$ and $\mathrm{F}$ analysis by ISE. 


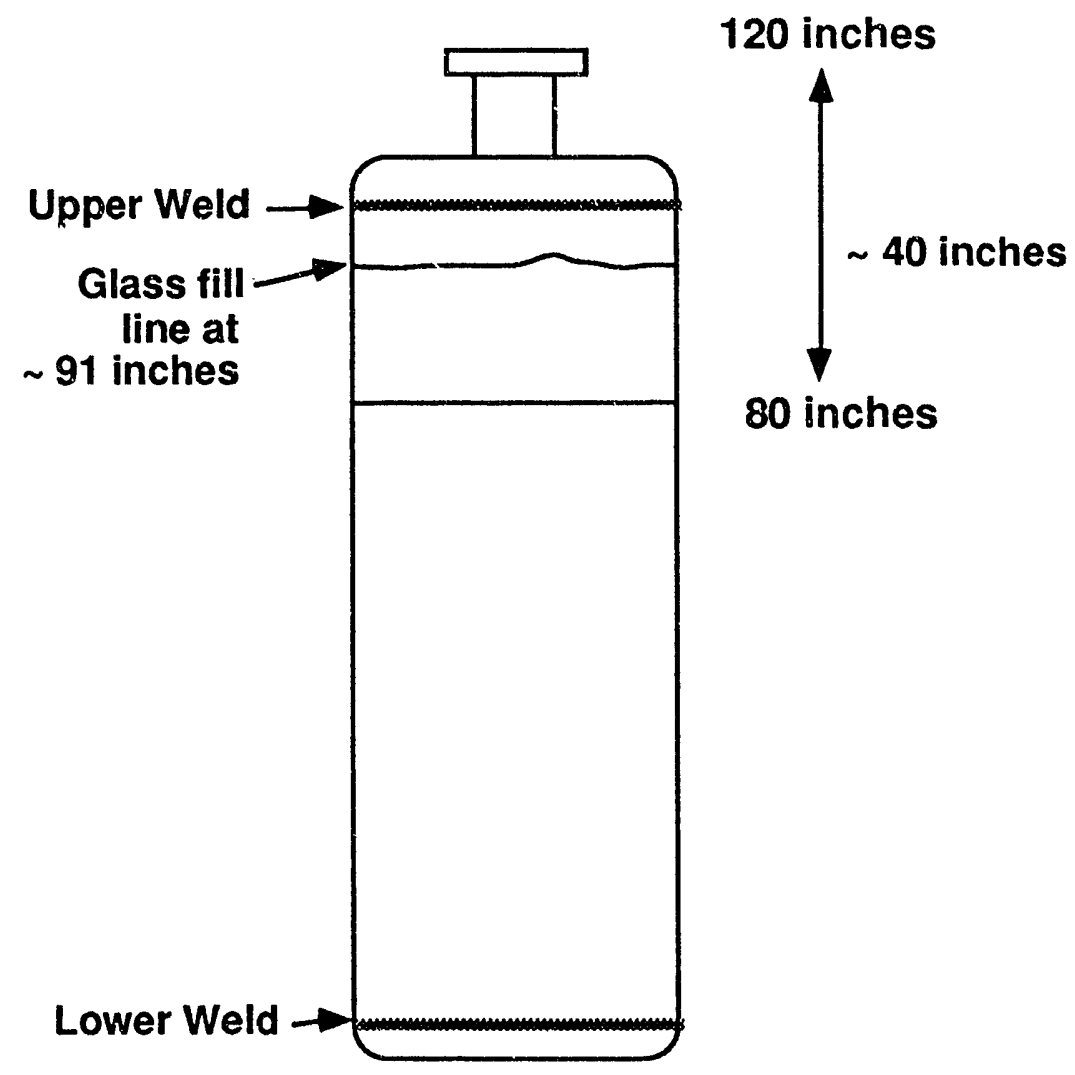

Figure 1 Schematic of full scale DWPF prototypic canister (Canister A-8, SGM 10-2) sectioned at 80 inch height for analysis of inorganic canister species. 


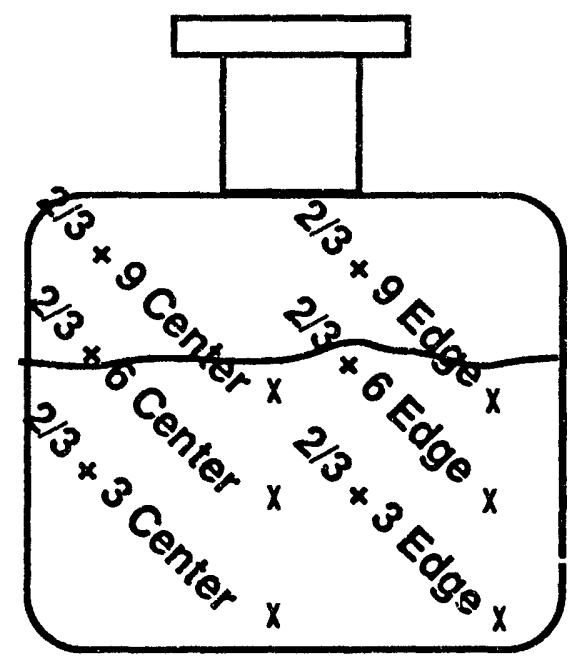

91 inches

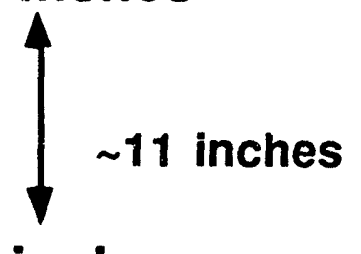

80 inches

Figure 2 Positions of six glass samples taken to examine the chemical homogeneity of the glass in Canister A-8 (SGM 10-2).

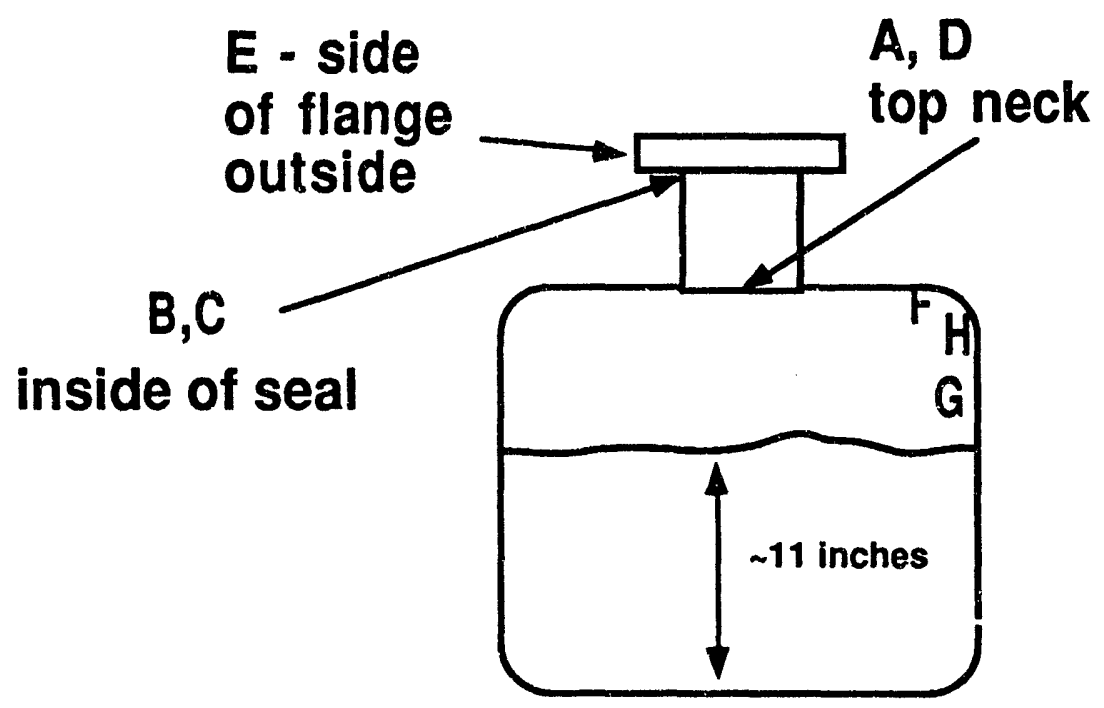

120 inches

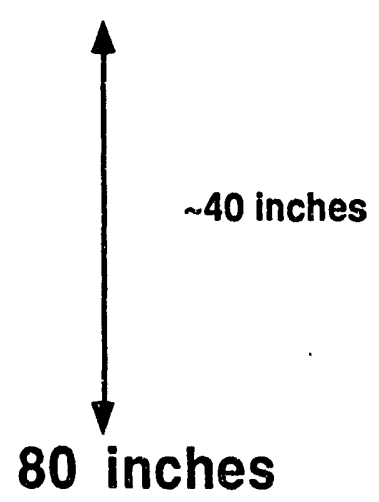

Figure 3 Position of eight deposit samples removed with tape for solids analysis in Canister A-8 (SGM 10-2). 


\section{X-ray Diffraction and Scanning Electron Microscopy Analyses}

$X$-ray diffraction (XRD) and Scanning Electron Microscopy (SEM) analyses coupled with Energy Dispersive Analysis by X-ray (EDAX) were performed on the 44 glass samples and the 8 tape samples in order to determine the phase and chemical composition of any deposits and/or reaction products. Dried solids from the water flush, were analyzed by XRD, SEM and EDAX as well.

\section{OUALITY ASSURANCE}

The SRTC Glass Technology Task Control Matrix for all the tasks in this study is given in Reference 15. All tasks were carried out in accordance with RW-0214 and NQA-1. Analytical Development Section (SRTC/ADS) procedures were followed for all chemical analyses.

Glass and deposit analyses are recorded in WSRC-NB-92-201. 


\section{RESULTS AND DISCUSSION}

\section{Glass Composition Analyses}

The analyses of the twelve glass samples taken from the top of canister SGM 10-2 are given on a normalized oxide basis in Table I. The anion analyses for $\mathrm{Cl}, \mathrm{F}$, and $\mathrm{SO}_{4}$ are given in element $w t \%$. The detailed chemical analyses are given in Appendix I. The maximum standard deviations on the twelve glass samples is $<1.0$ with the analyses for $\mathrm{Na}_{2} \mathrm{O}$ being the poorest, $\pm 0.94 \mathrm{wt} \%$ followed by the analyses for $\mathrm{SiO}_{2}, \pm 0.77 \mathrm{wt} \%$.

The average oxide composition of the SGM 10-2 glass is similar to that analyzed at the end of the SGM-10 Campaign when the melter was drained (Table I). ${ }^{16}$ The target alkali oxide/SiO 2 and $\mathrm{B}_{2} \mathrm{O}_{3} / \mathrm{SiO}_{2}$ ratios for the feed for the SGM 10 Campaign were 0.23 and 0.43 , respectively. 17 The corresponding ratios for the glass in canister SGM 10-2 were 0.21 and 0.42 . The ratios measured at the end of the SGM-10 Campaign were 0.23 and 0.37 .

The reference amount of chlorides and halides added to SGM 10 were based on the process constraints of $\mathrm{NaCl}<0.6 \mathrm{wt} \%, \mathrm{NaF}<0.1 \mathrm{wt} \%$ and $\mathrm{SO}_{4}{ }^{2-}<0.4 \mathrm{wt} \%$. This is equivalent to $\mathrm{Cl}<0.364 \mathrm{wt} \%, \mathrm{~F}<0.036$, and $\mathrm{SO}_{4}{ }^{2-}<0.4 \mathrm{wt} \%$. Table I indicates that the average concentrations of these species in the canister $10-2$ glass are $0.37 \mathrm{wt} \% \mathrm{Cl}, 0.02 \mathrm{wt} \% \mathrm{~F}$, and 0.16 $\mathrm{wt}_{\mathrm{SO}} \mathrm{SO}_{4}^{2-}$

The chemical analyses in Table I indicate that the SGM 10-2 canister glass was homogeneous. No chemical gradients in the oxides, halide, or sulfate compositions were observed when the compositions of the samples taken from the edge were compared to those taken from the center of the canister.

\section{Deposit and Reaction Product Analyses}

The analysis of the fifty two glass and deposit samples taken from canister SGM 10-2 are given in Table II. The small amounts of deposit material precluded any type of analysis but Scanning Electron Microscopy (SEM) coupled with quantitative elemental Energy Dispersive Analysis by Xray (EDAX). The stoichiometry of the phases observed during SEM analysis was determined by the similarly of these smaller deposits to larger deposits of the same chemistry found in the off-gas lines and in the cyclone separator of several SRS scale glass melters. ${ }^{7-9}$

Three deposit samples had sufficient volume that comparative X-ray Diffraction (XRD) and SEM analysis could be performed. The speciation determined by SEM was similar to that observed during XRD analysis (Table III). The samples that had been flushed from the top shoulder and sides of the canister interior and dried were analyzed by XRD and SEM (Table IV). There was insufficient liquid from the flushing to enable quantitative $\mathrm{Cl}$ and $\mathrm{F}$ analyses. 
Table 1. Composition of Glasses Sampled in Canister SGM-10-2

\begin{tabular}{|c|c|c|c|c|c|c|c|c|}
\hline $\begin{array}{l}\text { OXIDE } \\
\text { WT\% }\end{array}$ & $\begin{array}{l}2 / 3+0 \\
\text { EDGE-A } \\
* 200038840\end{array}$ & $\begin{array}{l}2 / 3+0 \\
\text { EDGE-B } \\
200038841\end{array}$ & $\begin{array}{l}2 / 3+0 \\
\text { CEN-A } \\
\$ 200038842\end{array}$ & $\begin{array}{l}2 / 3+0 \\
\text { CEN-B } \\
\$ 200038843\end{array}$ & $\begin{array}{l}2 / 3+6 \\
\text { EDGE-A } \\
\$ 200038844\end{array}$ & $\begin{array}{l}2 / 3+6 \\
\text { EDGE-B } \\
200038845\end{array}$ & $\begin{array}{l}2 / 3+6 \\
\text { CEN-A } \\
200038846\end{array}$ & $\begin{array}{r}2 / 3+6 \\
\text { CEN-B } \\
\# 200038847\end{array}$ \\
\hline $\mathrm{Al}_{2} \mathrm{O}_{3}$ & 3.55 & 3.13 & 3.55 & 3.13 & 5.38 & 3.45 & 3.40 & 3.32 \\
\hline $\begin{array}{l}\mathrm{CaO} \\
\mathrm{Fe}_{2} \mathrm{O}_{3}\end{array}$ & $\begin{array}{r}1.33 \\
11.15\end{array}$ & $\begin{array}{l}1.34 \\
9.69\end{array}$ & $\begin{array}{r}1.33 \\
11.15\end{array}$ & $\begin{array}{l}1.34 \\
9.69\end{array}$ & $\begin{array}{r}1.41 \\
10.62\end{array}$ & $\begin{array}{r}1.29 \\
10.90\end{array}$ & $\begin{array}{r}1.24 \\
10.88\end{array}$ & $\begin{array}{r}1.04 \\
10.74\end{array}$ \\
\hline $\begin{array}{l}\mathrm{MgO} \\
\mathrm{MnO} \\
\mathrm{Na}_{2} \mathrm{O}\end{array}$ & $\begin{array}{r}0.84 \\
2.19 \\
10.95\end{array}$ & $\begin{array}{r}0.85 \\
1.92 \\
11.99\end{array}$ & $\begin{array}{r}0.84 \\
2.19 \\
10.95\end{array}$ & $\begin{array}{r}0.85 \\
1.92 \\
11.99\end{array}$ & $\begin{array}{r}0.84 \\
2.10 \\
11.62\end{array}$ & $\begin{array}{r}0.85 \\
2.12 \\
11.72\end{array}$ & $\begin{array}{r}0.82 \\
2.03 \\
12.83\end{array}$ & $\begin{array}{r}0.80 \\
2.11 \\
13.61\end{array}$ \\
\hline $\mathrm{Li}_{2} \mathrm{O}$ & 4.47 & 4.61 & 4.47 & 4.61 & 4.37 & 4.52 & 4.40 & 4.38 \\
\hline $\begin{array}{l}\mathrm{NiO} \\
\mathrm{SiO}_{2}\end{array}$ & $\begin{array}{r}0.94 \\
50.84\end{array}$ & $\begin{array}{r}0.81 \\
51.65\end{array}$ & $\begin{array}{r}0.94 \\
50.84\end{array}$ & $\begin{array}{r}0.81 \\
51.65\end{array}$ & $\begin{array}{r}0.89 \\
49.54\end{array}$ & $\begin{array}{r}0.90 \\
50.61\end{array}$ & $\begin{array}{r}0.89 \\
49.98\end{array}$ & $\begin{array}{r}0.87 \\
49.59\end{array}$ \\
\hline $\mathrm{Cr}_{2} \mathrm{O}_{3}$ & 0.15 & 0.11 & 0.15 & 0.11 & 0.11 & 0.10 & 0.11 & 0.12 \\
\hline $\mathrm{B}_{2} \mathrm{O}_{3}$ & 12.07 & 12.35 & 12.07 & 12.35 & 11.67 & 12.04 & 11.94 & 11.73 \\
\hline $\begin{array}{l}\mathrm{SrO} \\
\mathrm{ZrO}_{2}\end{array}$ & $\begin{array}{l}0.04 \\
0.68\end{array}$ & $\begin{array}{l}0.05 \\
0.71\end{array}$ & $\begin{array}{l}0.04 \\
0.68\end{array}$ & $\begin{array}{l}0.05 \\
0.71\end{array}$ & $\begin{array}{l}0.05 \\
0.64\end{array}$ & $\begin{array}{l}0.04 \\
0.70\end{array}$ & $\begin{array}{l}0.04 \\
0.69\end{array}$ & $\begin{array}{l}0.04 \\
0.73\end{array}$ \\
\hline $\mathrm{TiO}_{2}$ & 0.15 & 0.14 & 0.15 & 0.14 & 0.14 & 0.13 & 0.14 & 0.16 \\
\hline $\mathrm{K}_{2} \mathrm{O}$ & 0.43 & 0.45 & 0.43 & 0.45 & 0.36 & 0.43 & 0.44 & 0.48 \\
\hline $\mathrm{P}_{2} \mathrm{O}_{5}$ & 0.10 & 0.07 & 0.10 & 0.07 & 0.14 & 0.05 & 0.03 & 0.04 \\
\hline $\mathrm{BaO}$ & 0.01 & 0.01 & 0.01 & 0.01 & 0.01 & 0.01 & 0.01 & 0.01 \\
\hline $\mathrm{ZnO}$ & 0.12 & 0.13 & 0.12 & 0.13 & 0.12 & 0.13 & 0.11 & 0.22 \\
\hline $\mathrm{Cl}$ & 0.46 & 0.41 & 0.46 & 0.41 & 0.39 & 0.43 & 0.35 & 0.33 \\
\hline $\mathrm{F}$ & 0.03 & 0.02 & 0.03 & 0.02 & 0.02 & 0.02 & 0.02 & 0.02 \\
\hline $\mathrm{SO}_{4}$ & 0.16 & 0.15 & 0.16 & 0.15 & 0.16 & 0.17 & 0.18 & 0.19 \\
\hline
\end{tabular}

$\begin{array}{lllllllll}\text { OXIDE } & 100.00 & 100.00 & 100.00 & 100.00 & 100.00 & 100.00 & 100.00 & 100.00\end{array}$

SUMS

MOLAR BALANCE (M)

$\begin{array}{lrrrrrrrr}\text { SALTS } & 1.11 & 1.04 & 1.11 & 1.04 & 0.94 & 1.05 & 0.92 & 0.96 \\ \text { OXIDES } & 98.89 & 98.96 & 98.89 & 98.96 & 99.06 & 98.95 & 99.08 & 99.04\end{array}$

\section{CALCUlated PARAMETERS}

\begin{tabular}{|c|c|c|c|c|c|c|c|c|}
\hline $\mathrm{ALK} / \mathrm{SiO}_{2}$ & 0.39 & 0.41 & 0.39 & 0.41 & 0.41 & 0.41 & 0.43 & 0.45 \\
\hline $\mathrm{B}_{2} \mathrm{O}_{3} / \mathrm{SiO}_{2}$ & 0.20 & 0.21 & 0.20 & 0.21 & 0.20 & 0.21 & 0.21 & 0.20 \\
\hline $\begin{array}{l}\text { VISCOSITY } \\
\text { (Poise at } 1150^{\circ}\end{array}$ & 43 & 38 & 43 & 38 & 45 & 37 & 31 & 27 \\
\hline $\begin{array}{l}\text { LIQUIDUS } \\
\left({ }^{\circ} \mathrm{C}\right)\end{array}$ & 949 & 911 & 949 & 911 & 959 & 944 & 947 & 945 \\
\hline
\end{tabular}


Table 1. Composition of Glasses Sampled in Canister SGM-10-2 (Cont'd)

\begin{tabular}{|c|c|c|c|c|c|c|c|}
\hline $\begin{array}{l}\text { OXIDE } \\
\text { WT\% }\end{array}$ & $\begin{array}{l}2 / 3+9 \\
\text { EDGE-A } \\
\$ 200038848\end{array}$ & $\begin{array}{l}2 / 3+9 \\
\text { EDGE-B } \\
200038849\end{array}$ & $\begin{array}{l}2 / 3+9 \\
\text { CEN-A } \\
\$ 200038850\end{array}$ & $\begin{array}{l}2 / 3+9 \\
\text { CEN-B } \\
\$ 200038851\end{array}$ & $\begin{array}{l}\text { AVERAGE } \\
\text { COMP }\end{array}$ & $\begin{array}{l}\text { STD } \\
\text { DEV }\end{array}$ & $\begin{array}{l}\text { SGM-10 } \\
\text { DRAIN } \\
\text { GLASS }\end{array}$ \\
\hline $\mathrm{Al}_{2} \mathrm{O}_{3}$ & 3.47 & 3.36 & 3.35 & 3.36 & 3.54 & 0.60 & 4.22 \\
\hline $\begin{array}{l}\mathrm{CaO} \\
\mathrm{Fe}_{2} \mathrm{O}_{3}\end{array}$ & $\begin{array}{r}1.06 \\
10.89\end{array}$ & $\begin{array}{r}1.01 \\
10.79\end{array}$ & $\begin{array}{r}0.96 \\
10.77\end{array}$ & $\begin{array}{r}1.27 \\
10.75\end{array}$ & $\begin{array}{r}1.22 \\
10.67\end{array}$ & $\begin{array}{l}0.15 \\
0.48\end{array}$ & $\begin{array}{r}1.41 \\
13.45\end{array}$ \\
\hline $\begin{array}{l}\mathrm{MgO} \\
\mathrm{MnO} \\
\mathrm{Na}_{2} \mathrm{O}\end{array}$ & $\begin{array}{r}0.84 \\
2.18 \\
11.54\end{array}$ & $\begin{array}{r}0.80 \\
2.11 \\
13.31\end{array}$ & $\begin{array}{r}0.77 \\
2.12 \\
13.31\end{array}$ & $\begin{array}{r}0.83 \\
2.09 \\
12.96\end{array}$ & $\begin{array}{r}0.83 \\
2.09 \\
12.23\end{array}$ & $\begin{array}{l}0.02 \\
0.09 \\
0.94\end{array}$ & $\begin{array}{r}0.60 \\
1.72 \\
12.85\end{array}$ \\
\hline $\mathrm{Li}_{2} \mathrm{O}$ & 4.48 & 4.40 & 4.37 & 4.42 & 4.46 & 0.09 & 4.02 \\
\hline $\begin{array}{l}\mathrm{NiO} \\
\mathrm{SiO}_{2}\end{array}$ & $\begin{array}{r}0.91 \\
50.99\end{array}$ & $\begin{array}{r}0.90 \\
49.81\end{array}$ & $\begin{array}{r}0.90 \\
49.64\end{array}$ & $\begin{array}{r}0.88 \\
50.03\end{array}$ & $\begin{array}{r}0.89 \\
50.43\end{array}$ & $\begin{array}{l}0.04 \\
0.77\end{array}$ & $\begin{array}{r}1.21 \\
47.28\end{array}$ \\
\hline $\mathrm{Cr}_{2} \mathrm{O}_{3}$ & 0.11 & 0.10 & 0.11 & 0.10 & 0.11 & 0.02 & 0.3 \\
\hline $\mathrm{B}_{2} \mathrm{O}_{3}$ & 12.04 & 11.90 & 12.13 & 11.84 & 12.01 & 0.21 & 11.04 \\
\hline $\begin{array}{l}\mathrm{SrO} \\
\mathrm{ZrO}_{2}\end{array}$ & $\begin{array}{l}0.04 \\
0.69\end{array}$ & $\begin{array}{l}0.04 \\
0.72\end{array}$ & $\begin{array}{l}0.04 \\
0.73\end{array}$ & $\begin{array}{l}0.04 \\
0.69\end{array}$ & $\begin{array}{l}0.04 \\
0.70\end{array}$ & $\begin{array}{l}0.00 \\
0.02\end{array}$ & $\begin{array}{l}0.3 \\
0.5\end{array}$ \\
\hline $\mathrm{TiO}_{2}$ & 0.13 & 0.13 & 0.14 & 0.13 & 0.14 & 0.01 & 0.3 \\
\hline $\mathrm{K}_{2} \mathrm{O}$ & 0.43 & 0.44 & 0.47 & 0.45 & 0.44 & 0.03 & 0.8 \\
\hline $\mathrm{P}_{2} \mathrm{O}_{5}$ & 0.06 & 0.04 & 0.04 & 0.03 & 0.06 & 0.03 & \\
\hline $\mathrm{BaO}$ & 0.01 & 0.01 & 0.01 & 0.00 & 0.01 & 0.00 & \\
\hline $\mathrm{ZnO}$ & 0.13 & 0.14 & 0.13 & 0.13 & 0.14 & 0.03 & \\
\hline $\mathrm{Cl}$ & 0.36 & 0.23 & 0.38 & 0.26 & 0.37 & 0.07 & \\
\hline$F$ & 0.02 & N/A & N/A & N/A & 0.02 & 0.00 & \\
\hline $\mathrm{SO}_{4}$ & 0.17 & 0.15 & 0.11 & 0.18 & 0.16 & 0.02 & \\
\hline
\end{tabular}

$\begin{array}{lllllll}\text { OXIDE } & 100.00 & 100.00 & 100.00 & 100.00 & 100.00 & 100.00\end{array}$

SUMS

MOLAR BALANCE (M)

$\begin{array}{lrrrrr}\text { SALTS } & 0.97 & 0.67 & 0.87 & 0.74 & 0.95 \\ \text { OXIDES } & 99.03 & 99.33 & 99.13 & 99.26 & 99.05\end{array}$

CAlCulated PARAMETERS

\begin{tabular}{|c|c|c|c|c|c|c|}
\hline $\mathrm{ALK} / \mathrm{SiO}_{2}$ & 0.40 & 0.44 & 0.44 & 0.43 & 0.42 & 0.37 \\
\hline $\mathrm{B}_{2} \mathrm{O}_{3} / \mathrm{SiO}_{2}$ & 0.20 & 0.21 & 0.21 & 0.20 & 0.21 & 0.23 \\
\hline $\begin{array}{l}\text { VISCOSITY } \\
\text { (Poise at } 1150^{\circ}\end{array}$ & C) ${ }^{40}$ & 29 & 28 & 31 & 36 & \\
\hline $\begin{array}{l}\text { LIQUIDUS } \\
\left({ }^{\circ} \mathrm{C}\right)\end{array}$ & 942 & 946 & 946 & 943 & 941 & \\
\hline
\end{tabular}


Table II. Phase Identification of Canister Deposits.

\section{$\begin{array}{ll}\text { Sample } & \text { Sample } \\ \text { ID } & \text { Identification }\end{array}$}

A Top of neck of canister

$\begin{array}{ll}\text { B } & \text { Inside of Seal } \\ \text { C } & \text { Inside of Seal } \\ \text { D } & \text { Top of neck of canister }\end{array}$

E Outside of Flange

F Inside Neck of canister

\section{SEM Analysis}

spinel/rust $(\mathrm{Fe}, \mathrm{Ni}, \mathrm{Cr})$

glass ( $\mathrm{Si}, \mathrm{Al}, \mathrm{Fe}, \mathrm{Ca}, \mathrm{etc})$

$(\mathrm{Na}, \mathrm{K}) \mathrm{Cl}$

$(\mathrm{Na}, \mathrm{K})_{2} \mathrm{SO}_{4}$

$\mathrm{NaF}$ phase

spinel/rust (Fe, Ni, Cr)

$(\mathrm{Na}, \mathrm{K}) \mathrm{Cl}$

$(\mathrm{Na}, \mathrm{K})_{2} \mathrm{SO}_{4}$

spinel/rust (Fe, $\mathrm{Ni}, \mathrm{Cr}$ )

trace of glass ( $\mathrm{Si}, \mathrm{Al}, \mathrm{Fe}, \mathrm{Ca}, \mathrm{etc})$

trace of $(\mathrm{Na}, \mathrm{K}) \mathrm{Cl}$

trace of $(\mathrm{Na}, \mathrm{K})_{2} \mathrm{SO}_{4}$

spinel/rust (Fe, Ni, Cr)

glass ( $\mathrm{Si}, \mathrm{Al}, \mathrm{Fe}, \mathrm{Ca}, \mathrm{etc})$

$(\mathrm{Na}, \mathrm{K}) \mathrm{Cl}$

$(\mathrm{Na}, \mathrm{K})_{2} \mathrm{SO}_{4}$

$\mathrm{NaF}$ phase

$(\mathrm{Na}, \mathrm{K}) \mathrm{Cl}$

$(\mathrm{Na}, \mathrm{K})_{2} \mathrm{SO}_{4}$

- $\quad \mathrm{Sn}, \mathrm{Ti}, \mathrm{Zn}$ from cutting fluid/blade

rust (high $\mathrm{Fe}, \mathrm{Ni}, \mathrm{Cr}$ )

trace of $\mathrm{B}$ as $\mathrm{Na}_{2} \mathrm{~B}_{4} \mathrm{O}_{7}$

trace of $(\mathrm{Na}, \mathrm{K}) \mathrm{Cl}$

trace of $(\mathrm{Na}, \mathrm{K})_{2} \mathrm{SO}_{4}$

$\mathrm{Ti}$ from cutting fluid/blade

Sn, Ti, Zn from cutting fluid/blade spinel/rust (Fe, $\mathrm{Ni}, \mathrm{Cr}$ )

$(\mathrm{Na}, \mathrm{K}) \mathrm{Cl}$

$(\mathrm{Na}, \mathrm{K})_{2} \mathrm{SO}_{4}$

$\mathrm{NaF}$ phase 
Table II. Phase Identification of Canister Deposits (continued).

\begin{tabular}{ll}
$\begin{array}{l}\text { Sample } \\
\text { ID }\end{array}$ & \multicolumn{1}{c}{$\begin{array}{c}\text { Sample } \\
\text { Identification }\end{array}$} \\
H & $\begin{array}{l}\text { White streaky deposit } \\
\text { on side of canister above } \\
\text { glass melt line }\end{array}$ \\
I & $\begin{array}{l}\text { Smear of bottom of } \\
\text { inner canister closure plug }\end{array}$ \\
& J \\
& $\begin{array}{l}\text { Gray powder from glass } \\
\text { surface }\end{array}$
\end{tabular}

K White deposits near ylass pour surface

\section{SEM Analysis}

spinel/rust (Fe, $\mathrm{Ni}, \mathrm{Cr}$ )

Ai, Si rich material

$\mathrm{Zn}$ from cutting fluid/blade

glass ( $\mathrm{Si}, \mathrm{Al}, \mathrm{Fe}, \mathrm{Ca}$, etc)

$(\mathrm{Na}, \mathrm{K}) \mathrm{Cl}$

$(\mathrm{Na}, \mathrm{K})_{2} \mathrm{SO}_{4}$

trace spinel/rust $(\mathrm{Fe}, \mathrm{Ni}, \mathrm{Cr}$ )

trace of $(\mathrm{Na}, \mathrm{K}) \mathrm{Cl}$

trace of $(\mathrm{Na}, \mathrm{K})_{2} \mathrm{SO}_{4}$

W, Ti, Zn from cutting fluid/blade glass ( $\mathrm{Si}, \mathrm{Al}, \mathrm{Fe}, \mathrm{Ca}, \mathrm{etc})$

trace of $(\mathrm{Na}, \mathrm{K}) \mathrm{Cl}$

trace of $(\mathrm{Na}, \mathrm{K})_{2} \mathrm{SO}_{4}$

glass ( $\mathrm{Si}, \mathrm{Al}, \mathrm{Fe}, \mathrm{Ca}$, etc)

$(\mathrm{Na}, \mathrm{K}) \mathrm{Cl}$

$(\mathrm{Na}, \mathrm{K})_{2} \mathrm{SO}_{4}$

$\mathrm{NaF}$ phase

$\mathrm{Zn}$ from cutting fluid/blade

glass ( $\mathrm{Si}, \mathrm{Al}, \mathrm{Fe}, \mathrm{Ca}$, etc)

$(\mathrm{Na}, \mathrm{K}) \mathrm{Cl}$

spinel/rust (Fe, Ni, Cr)

$\mathrm{W}, \mathrm{Ti}, \mathrm{Zn}$ from cutting fluid/blade

M Top of Pour Surface

glass ( $\mathrm{Si}, \mathrm{Al}, \mathrm{Fe}, \mathrm{Ca}$, etc)

glass ( $\mathrm{Si}, \mathrm{Al}, \mathrm{Fe}, \mathrm{Ca}$, etc)

$(\mathrm{Ca}, \mathrm{K})_{2} \mathrm{SO}_{4}$

$\mathrm{Zn}$ from cutting fluid/blade

glass ( $\mathrm{Si}, \mathrm{Al}, \mathrm{Fe}, \mathrm{Ca}, \mathrm{etc}$ )

spinel/rust (Fe, $\mathrm{Ni}, \mathrm{Cr}$ ) 
Table II. Phase Identification of Canister Deposits (continued).

$\begin{array}{ll}\text { Sample } & \begin{array}{l}\text { Sample } \\ \text { Identification }\end{array} \\ \mathrm{P} & \begin{array}{l}\text { Brown deposit on canister } \\ \text { side below melt surface }\end{array} \\ \mathrm{Q} & \begin{array}{l}\text { Glass with large bubbles } \\ \mathrm{R}\end{array} \\ & \begin{array}{l}\text { "Pock" mark on side of } \\ \text { canister below melt } \\ \text { surface }\end{array} \\ \mathrm{S} & \begin{array}{l}\text { "Blister" on side of canister } \\ \text { below melt surface }\end{array} \\ \end{array}$

T Deposits from "wet" glass

U Brown deposit on glass

V, W Deposits on glass

X Deposits on top glass surface

\section{SEM Analysis}

glass ( $\mathrm{Si}, \mathrm{Al}, \mathrm{Fe}, \mathrm{Ca}$, etc)

$(\mathrm{Na}, \mathrm{K}) \mathrm{Cl}$

spinel/rust ( $\mathrm{Fe}, \mathrm{Ni}, \mathrm{Cr}$ )

$\mathrm{B}$ (either glass or $\mathrm{Na}_{2} \mathrm{~B}_{4} \mathrm{O}_{7}$ )

$\mathrm{Ti}, \mathrm{Zn}$ from cutting fluid/blade

glass ( $\mathrm{Si}, \mathrm{Al}, \mathrm{Fe}, \mathrm{Ca}$, etc)

spinel/rust (Fe, $\mathrm{Ni}, \mathrm{Cr}$ )

$\mathrm{Ti}, \mathrm{Zn}$ from cutting fluid/blade

$\mathrm{Sn}, \mathrm{Ti}, \mathrm{Zn}$ from cutting fluid/blade a lot of dendritic $(\mathrm{Na}, \mathrm{K}) \mathrm{Cl}$ trace of $(\mathrm{Na}, \mathrm{K})_{2} \mathrm{SO}_{4}$ spinel/rust ( $\mathrm{Fe}, \mathrm{Ni}, \mathrm{Cr}$ ) spinel crystals (Fe, $\mathrm{Ni}, \mathrm{Cr}$ )

glass ( $\mathrm{Si}, \mathrm{Al}, \mathrm{Fe}, \mathrm{Ca}$, etc)

$(\mathrm{Na}, \mathrm{K}) \mathrm{Cl}$

$(\mathrm{Na}, \mathrm{K})_{2} \mathrm{SO}_{4}$

glass ( $\mathrm{Si}, \mathrm{Al}, \mathrm{Fe}, \mathrm{Ca}$, etc)

$(\mathrm{Na}, \mathrm{K}) \mathrm{Cl}$

$(\mathrm{Na}, \mathrm{K})_{2} \mathrm{SO}_{4}$

glass ( $\mathrm{Si}, \mathrm{Al}, \mathrm{Fe}, \mathrm{Ca}, \mathrm{etc})$

$(\mathrm{Na}, \mathrm{K}) \mathrm{Cl}$

$(\mathrm{Na}, \mathrm{K})_{2} \mathrm{SO}_{4}$

$\mathrm{NaF}$ phase

glass ( $\mathrm{Si}, \mathrm{Al}, \mathrm{Fe}, \mathrm{Ca}$, etc)

$\mathrm{Zn}$ from cutting fluid/blade

glass ( $\mathrm{Si}, \mathrm{Al}, \mathrm{Fe}, \mathrm{Ca}$, etc)

$(\mathrm{Na}, \mathrm{K}) \mathrm{Cl}$

$(\mathrm{Na}, \mathrm{K})_{2} \mathrm{SO}_{4}$

Ti from cutting fluid/blade 
Table II. Phase Identification of Canister Deposits (continued).

\begin{tabular}{|c|c|c|}
\hline $\begin{array}{l}\text { Sample } \\
\text { ID }\end{array}$ & $\begin{array}{l}\text { Sample } \\
\text { Identification }\end{array}$ & SEM Analysis \\
\hline Y & Deposits on top glass surface & $\begin{array}{l}\text { glass (Si, } \mathrm{Al}, \mathrm{Fe}, \mathrm{Ca}, \mathrm{etc}) \\
(\mathrm{Na}, \mathrm{K}) \mathrm{Cl} \\
(\mathrm{Na}, \mathrm{K})_{2} \mathrm{SO}_{4} \\
\text { spinel/rust (Fe, Ni, Cr) } \\
\text { Sn,Ti from cutting fluid/blade }\end{array}$ \\
\hline $\mathrm{Z}$ & Deposits on top glass surface & $\begin{array}{l}\text { glass }(\mathrm{Si}, \mathrm{Al}, \mathrm{Fe}, \mathrm{Ca}, \text { etc }) \\
(\mathrm{Na}, \mathrm{K}) \mathrm{Cl} \\
(\mathrm{Na}, \mathrm{K})_{2} \mathrm{SO}_{4} \\
\text { spinel/rust }(\mathrm{Fe}, \mathrm{Ni}, \mathrm{Cr})\end{array}$ \\
\hline AA & Deposits on top glass surface & $\begin{array}{l}\text { glass ( } \mathrm{Si}, \mathrm{Al}, \mathrm{Fe}, \mathrm{Ca}, \mathrm{etc}) \\
\text { dendritic }(\mathrm{Na}, \mathrm{K}) \mathrm{Cl} \\
\text { spinel/rust }(\mathrm{Fe}, \mathrm{Ni}, \mathrm{Cr})\end{array}$ \\
\hline $\mathrm{BB}$ & $\begin{array}{l}\text { Deposits on top glass surface } \\
\text { with visible powdery deposits }\end{array}$ & $\begin{array}{l}\text { glass ( } \mathrm{Si}, \mathrm{Al}, \mathrm{Fe}, \mathrm{Ca}, \mathrm{etc}) \\
\text { spinel/rust (Fe, Ni, Cr) }\end{array}$ \\
\hline $\mathrm{CC}$ & Melt pour surface & $\begin{array}{l}\text { glass }(\mathrm{Si}, \mathrm{Al}, \mathrm{Fe}, \mathrm{Ca}, \mathrm{etc}) \\
(\mathrm{Na}, \mathrm{K}) \mathrm{Cl} \\
(\mathrm{Na}, \mathrm{K})_{2} \mathrm{SO}_{4}\end{array}$ \\
\hline DD & $\begin{array}{l}\text { White deposit embedded in } \\
\text { glass }\end{array}$ & $\begin{array}{l}\text { Na aluminosilicate } \\
\text { W from cutting fluid/blade }\end{array}$ \\
\hline $\mathrm{EE}, \mathrm{FF}$ & Melt pour surface & $\begin{array}{l}\text { glass ( } \mathrm{Si}, \mathrm{Al}, \mathrm{Fe}, \mathrm{Ca} \text {, etc) } \\
\mathrm{W}, \mathrm{Ti}, \mathrm{Zn} \text { from cutting fluid/blade } \\
(\mathrm{Na}, \mathrm{K}) \mathrm{Cl} \\
(\mathrm{Na}, \mathrm{K})_{2} \mathrm{SO}_{4}\end{array}$ \\
\hline GG & $\begin{array}{l}\text { Clean glass fracture surface } \\
\text { at top of canister }\end{array}$ & glass ( $\mathrm{Si}, \mathrm{Al}, \mathrm{Fe}, \mathrm{Ca}, \mathrm{etc})$ \\
\hline $\mathrm{HH}$ & $\begin{array}{l}\text { Glass fracture surface with } \\
\text { brown deposits }\end{array}$ & $\begin{array}{l}\text { glass }(\mathrm{Si}, \mathrm{Al}, \mathrm{Fe}, \mathrm{Ca}, \mathrm{etc}) \\
\text { cubic }(\mathrm{Na}, \mathrm{K}) \mathrm{Cl} \text { crystals } \\
(\mathrm{Na}, \mathrm{K})_{2} \mathrm{SO}_{4} \\
\text { NaF phase }\end{array}$ \\
\hline
\end{tabular}


Table II. Phase Identification of Canister Deposits (continued).

$\begin{array}{ll}\begin{array}{l}\text { Sample } \\ \text { ID }\end{array} & \begin{array}{c}\text { Sample } \\ \text { Identification }\end{array} \\ \text { II } & \begin{array}{l}\text { Glass fracture surface at } \\ \text { top of canister }\end{array} \\ \text { JJ } & \begin{array}{l}\text { Opposite side of fracture } \\ \text { surface in II }\end{array}\end{array}$

KK Glass fracture surface at top of canister

LL Glass fracture surface at top of canister

MM Glass fracture surface at top of canister with brown deposits

NN, OO Glass fracture with "blister"

PP Elliptical bubbles (vugs)

\section{SEM Analysis}

glass ( $\mathrm{Si}, \mathrm{Al}, \mathrm{Fe}, \mathrm{Ca}$, etc)

$(\mathrm{Na}, \mathrm{K}) \mathrm{Cl}$

$(\mathrm{Na}, \mathrm{K})_{2} \mathrm{SO}_{4}$

$\mathrm{NaF}$ phase

Sn, Ti from cutting fluid

glass ( $\mathrm{Si}, \mathrm{Al}, \mathrm{Fe}, \mathrm{Ca}$, etc)

$(\mathrm{Na}, \mathrm{K}) \mathrm{Cl}$

$(\mathrm{Na}, \mathrm{K})_{2} \mathrm{SO}_{4}$

NaF phase

$\mathrm{Sn}, \mathrm{Ti}, \mathrm{Ni}, \mathrm{Cu}, \mathrm{W}$ from cutting fluid glass ( $\mathrm{Si}, \mathrm{Al}, \mathrm{Fe}, \mathrm{Ca}, \mathrm{etc})$

$(\mathrm{Na}, \mathrm{K}) \mathrm{Cl}$

$(\mathrm{Na}, \mathrm{K})_{2} \mathrm{SO}_{4}$

glass ( $\mathrm{Si}, \mathrm{Al}, \mathrm{Fe}, \mathrm{Ca}$, etc)

Sn from cutting fluid

spinel/rust (Fe, Ni, Cr)

$(\mathrm{Na}, \mathrm{K}) \mathrm{Cl}$

$(\mathrm{Na}, \mathrm{K})_{2} \mathrm{SO}_{4}$

W, Sn, Ti from cutting fluid/blade

glass ( $\mathrm{Si}, \mathrm{Al}, \mathrm{Fe}, \mathrm{Ca}$, etc)

spinel/rust (Fe, Ni, Cr)

$(\mathrm{Na}, \mathrm{K}) \mathrm{Cl}$

$(\mathrm{Na}, \mathrm{K})_{2} \mathrm{SO}_{4}$

spinel crystals in small vugs $(\mathrm{Fe}, \mathrm{Ni}, \mathrm{Cr})$

rust $(\mathrm{Fe}, \mathrm{Ni}, \mathrm{Cr}$ )

glass ( $\mathrm{Si}, \mathrm{Al}, \mathrm{Fe}, \mathrm{Ca}, \mathrm{etc})$

trace $(\mathrm{Na}, \mathrm{K}) \mathrm{Cl}$

trace $(\mathrm{Na}, \mathrm{K})_{2} \mathrm{SO}_{4}$

glass ( $\mathrm{Si}, \mathrm{Al}, \mathrm{Fe}, \mathrm{Ca}$, etc)

spinel/rust (Fe, Ni, Cr)

$(\mathrm{Na}, \mathrm{K}) \mathrm{Cl}$

$(\mathrm{Na}, \mathrm{K})_{2} \mathrm{SO}_{4}$ 
Table II. Phase Identification of Canister Deposits (continued).

$\begin{array}{ll}\begin{array}{l}\text { Sample } \\ \text { ID }\end{array} & \begin{array}{l}\text { Sample } \\ \text { Identification }\end{array} \\ \text { QQ } & \begin{array}{l}\text { Tape smear of melt pour } \\ \text { surface }\end{array}\end{array}$

RR Miscellaneous deposits

SS Clean glass fracture surface at top of canister

TT Deposits on interior shoulder of canister

UU Glass fracture surface at top of canister

VV White deposits on glass stringer near interior shoulder of canister

WW Glass fracture surface at top of canister

\section{SEM Analysis}

spinel/rust (Fe, $\mathrm{Ni}, \mathrm{Cr}$ )

glass ( $\mathrm{Si}, \mathrm{Al}, \mathrm{Fe}, \mathrm{Ca}$, etc)

$(\mathrm{Na}, \mathrm{K}) \mathrm{Cl}$

$(\mathrm{Na}, \mathrm{K})_{2} \mathrm{SO}_{4}$

Sr. from cutting fluid/blade glass ( $\mathrm{Si}, \mathrm{Al}, \mathrm{Fe}, \mathrm{Ca}$, etc) spinel/rust ( $\mathrm{Fe}, \mathrm{Ni}, \mathrm{Cr}$ )

$(\mathrm{Na}, \mathrm{K}) \mathrm{Cl}$

$(\mathrm{Na}, \mathrm{K})_{2} \mathrm{SO}_{4}$

glass ( $\mathrm{Si}, \mathrm{Al}, \mathrm{Fe}, \mathrm{Ca}$, etc) spinel crystals (Fe, $\mathrm{Ni}, \mathrm{Cr}$ ) Sn from cutting fluid/blade $(\mathrm{Na}, \mathrm{K}) \mathrm{Cl}$ $(\mathrm{Na}, \mathrm{K})_{2} \mathrm{SO}_{4}$

glass ( $\mathrm{Si}, \mathrm{Al}, \mathrm{Fe}, \mathrm{Ca}$, etc) $\mathrm{W}, \mathrm{Cu}$ from cutting fluid/blade $(\mathrm{Na}, \mathrm{K}) \mathrm{Cl}$ (immiscible in $\mathrm{SO}_{4}$ phase)

$(\mathrm{Na}, \mathrm{K})_{2} \mathrm{SO}_{4}$ (immiscible in $\mathrm{Cl}$ phase)

glass ( $\mathrm{Si}, \mathrm{Al}, \mathrm{Fe}, \mathrm{Ca}, \mathrm{etc})$

$\mathrm{W}$, Ti from cutting fluid/blade spinel/rust (Fe, Ni, Cr)

$(\mathrm{Na}, \mathrm{K}) \mathrm{Cl}$ (immiscible in $\mathrm{SO}_{4}$ phase)

$(\mathrm{Na}, \mathrm{K})_{2} \mathrm{SO}_{4}$ (immiscible in $\mathrm{Cl}$ phase)

glass ( $\mathrm{Si}, \mathrm{Al}, \mathrm{Fe}, \mathrm{Ca}$, etc)

Sn from cutting fluid/blade

large cubic $(\mathrm{Na}, \mathrm{K}) \mathrm{Cl}$ crystals

$(\mathrm{Na}, \mathrm{K})_{2} \mathrm{SO}_{4}$

glass ( $\mathrm{Si}, \mathrm{Al}, \mathrm{Fe}, \mathrm{Ca}$, etc)

Sn,Ti from cutting fluid/blade

spinel/rust (Fe, $\mathrm{Ni}, \mathrm{Cr}$ )

$(\mathrm{Na}, \mathrm{K}) \mathrm{Cl}$

$(\mathrm{Na}, \mathrm{K})_{2} \mathrm{SO}_{4}$ 
Table II. Phase Identification of Canister Deposits (continued).

\begin{tabular}{|c|c|c|}
\hline $\begin{array}{l}\text { Sample } \\
\text { ID }\end{array}$ & $\begin{array}{l}\text { Sample } \\
\text { Identification }\end{array}$ & SEM Analysis \\
\hline $\mathrm{XX}$ & Fractured melt pour surface & $\begin{array}{l}\text { glass }(\mathrm{Si}, \mathrm{Al}, \mathrm{Fe}, \mathrm{Ca}, \text { etc) } \\
\text { Ti from cutting fluid/blade } \\
\text { spinel/rust }(\mathrm{Fe}, \mathrm{Ni}, \mathrm{Cr}) \\
(\mathrm{Na}, \mathrm{K}) \mathrm{Cl} \\
\text { trace }(\mathrm{Na}, \mathrm{K})_{2} \mathrm{SO}_{4}\end{array}$ \\
\hline YY & $\begin{array}{l}\text { Glass in contact with canister } \\
\text { sidewall }\end{array}$ & $\begin{array}{l}\text { glass ( } \mathrm{Si}, \mathrm{Al}, \mathrm{Fe}, \mathrm{Ca}, \mathrm{etc}) \\
\text { spinel/rust }(\mathrm{Fe}, \mathrm{Ni}, \mathrm{Cr}) \\
(\mathrm{Na}, \mathrm{K}) \mathrm{Cl} \\
\text { trace }(\mathrm{Na}, \mathrm{K})_{2} \mathrm{SO}_{4}\end{array}$ \\
\hline $\mathrm{ZZ}$ & Bubbles (vugs) on melt surface & $\begin{array}{l}\text { glass ( } \mathrm{Si}, \mathrm{Al}, \mathrm{Fe}, \mathrm{Ca}, \text { etc) } \\
\text { spinel crystals }(\mathrm{Fe}, \mathrm{Ni}, \mathrm{Cr}) \\
(\mathrm{Na}, \mathrm{K}) \mathrm{Cl} \\
\text { trace }(\mathrm{Na}, \mathrm{K})_{2} \mathrm{SO}_{4}\end{array}$ \\
\hline
\end{tabular}


Table III Comparison of Scanning Electron Microscopy (SEM) and X-ray Diffraction (XRD) Deposit Identification.

\begin{tabular}{|c|c|c|c|}
\hline $\begin{array}{l}\text { Sample } \\
\text { ID }\end{array}$ & $\begin{array}{l}\text { Sample } \\
\text { Identification }\end{array}$ & SEM Analysis & XRD Analysis \\
\hline $\mathrm{E}$ & Outside of Flange & $\begin{array}{l}(\mathrm{Na}, \mathrm{K}) \mathrm{Cl} \\
(\mathrm{Na}, \mathrm{K})_{2} \mathrm{SO}_{4} \\
\mathrm{Sn}, \mathrm{Ti}, \mathrm{Zn} \text { from } \\
\text { cutting fluid/blade }\end{array}$ & $\begin{array}{l}\mathrm{Al}_{2} \mathrm{Si}_{2} \mathrm{O}_{5}(\mathrm{OH})_{4} \\
\mathrm{~KB}_{3} \mathrm{O}_{5} \\
\mathrm{ZnO}, \mathrm{TiO}_{2}\end{array}$ \\
\hline $\mathrm{F}$ & $\begin{array}{l}\text { Inside iveck of canister } \\
\text { "rust" }\end{array}$ & $\begin{array}{l}\text { rust (high } \mathrm{Fe}, \mathrm{Ni}, \mathrm{Cr} \text { ) } \\
\text { trace of } \mathrm{B} \text { as } \mathrm{Na}_{2} \mathrm{~B}_{4} \mathrm{O} \\
\text { trace of }(\mathrm{Na}, \mathrm{K}) \mathrm{Cl} \\
\text { trace of }(\mathrm{Na}, \mathrm{K})_{2} \mathrm{SO}_{4} \\
\text { Ti from cutting fluid/blade }\end{array}$ & $\begin{array}{l}\mathrm{Fe}_{2} \mathrm{O}_{3} \\
\mathrm{~KB}_{3} \mathrm{O}_{5}\end{array}$ \\
\hline $\mathrm{H}$ & $\begin{array}{l}\text { White streaky deposit } \\
\text { on side of canister above } \\
\text { glass melt line }\end{array}$ & $\begin{array}{l}\text { spinel/rust (Fe, } \mathrm{Ni}, \mathrm{Cr}) \\
\mathrm{Al}, \mathrm{Si} \text { rich material } \\
\mathrm{Zn} \text { from cutting fluid/blade } \\
\text { glass }(\mathrm{Si}, \mathrm{Al}, \mathrm{Fe}, \mathrm{Ca}, \text { etc) } \\
(\mathrm{Na}, \mathrm{K}) \mathrm{Cl} \\
(\mathrm{Na}, \mathrm{K})_{2} \mathrm{SO}_{4}\end{array}$ & amorphous \\
\hline
\end{tabular}


Table IV Liquid Samples Flushed from Side Walls of Canister

$\begin{array}{lll} & \text { SEM } & \text { XRD } \\ \text { Sample Identification } & \text { Analysis } & \text { Analysis }\end{array}$

Flush Top Solid

glass ( $\mathrm{Si}, \mathrm{Mn}, \mathrm{Fe})$

$(\mathrm{Na}, \mathrm{K}) \mathrm{Cl}$

Insufficient

$(\mathrm{Na}, \mathrm{K}) \mathrm{SO}_{4}$

Flush Side Solid

giass $(\mathrm{Si}, \mathrm{Fe}, \mathrm{Mn}, \mathrm{Ni}, \mathrm{Cr}) \quad \mathrm{NaCl}$
spinel/rust $(\mathrm{Fe}, \mathrm{Mn}, \mathrm{Ni}, \mathrm{Cr})$
$(\mathrm{Na}, \mathrm{K}) \mathrm{Cl}$
$(\mathrm{Na}, \mathrm{K}) \mathrm{SO}_{4}$


The off-gas line and cyclone separator deposits of scale glass melters at SRS were shown to form by vapor phase transport and condensation. ${ }^{7-9}$ Vaporization of species from the melt as it enters the canister and condensation on the interior canister shoulder and neck as the glass is poured is the most likely mechanism by which halide and sulfate species could be deposited in these regions of the canister. The vapor phase transported phases observed in the off-gas line deposits were identified by XRD and include the following:

- $\quad$ Sodium/potassium/cesium salts

$(\mathrm{Na}, \mathrm{K}, \mathrm{Cs})_{2} \mathrm{SO}_{4}$

$(\mathrm{Na}, \mathrm{K}, \mathrm{Cs}) \mathrm{Cl}$

$(\mathrm{Na}, \mathrm{K}, \mathrm{Cs}) \mathrm{BF}_{4}$

- $\quad$ Sodium/potassium borate

$(\mathrm{Na}, \mathrm{K})_{2} \mathrm{~B}_{4} \mathrm{O}$

- $\quad$ Sodium/potassium chromates

$(\mathrm{Na}, \mathrm{K})_{2} \mathrm{CrO}_{4}$

Similar $(\mathrm{Na}, \mathrm{K})_{2} \mathrm{SO}_{4}$ and $(\mathrm{Na}, \mathrm{K}) \mathrm{Cl}$ deposits were identified on the samples of tape which had been used to remove deposits from the shoulder and side of the interior of canister SGM 10-2 above the glass fill line (Samples A through H; Table II, III, Figure 3). An NaF phase presumed to be $(\mathrm{Na}, \mathrm{K})_{2} \mathrm{BF}_{4}$ was also identified in many of these samples. The positive identification of this phase is complicated by the fact that the conventional SEM/EDAX detector is not sensitive to elements as light in atomic weight as $\mathrm{B}$. Hence, the EDAX spectra for $\mathrm{NaF}$ and $\mathrm{Na}_{2} \mathrm{BF}_{4}$ appear identicai. Positive identification of a borate phase in Sample F from the inside neck of the canister was made by XRD (Table III). Reanalysis of sample F by EDAX with a wide angle detector indicated that $\mathrm{B}$ was, indeed, present (Table II). The phase was assumed to be $\left(\mathrm{Na}, \mathrm{K}_{2} \mathrm{~B}_{4} \mathrm{O}_{7}\right.$ as identified in the off-gas line deposits. Distribution of $(\mathrm{Na}, \mathrm{K})_{2} \mathrm{~B}_{4} \mathrm{O}_{7}$ may be more ubiquitous but the presence of this phase is difficult to establish due to its amorphous nature upon condensation. Thie only other borate deposits noted on the interior walls of the canister above the melt line were traces of shards of glass.

Alkaii chromates were not identified on the interior of the SGM 10-2 canister as they were in the SGM off-gas line deposits. The presence of alkali chromates in the off-gas line was complicated by evidence that the deposits were formed by reaction of the other alkali salts with chromium from the Inconel 690 off-gas pipe. Chromate deposits may, therefore not form when the other alkali salts interact with 304L stainless steel. The chromium rich phases that were detected during EDAX analysis of the SGM 10-2 canister deposits were primarily spinel crystals and/or $\mathrm{Fe}-\mathrm{Cr}-\mathrm{Ni}$ rich deposits of rust. However, the presence of alkali chromates as condensates in the canister interior can not be precluded.

Rust was visibly present on the shoulder and side of the interior of canister SGM 10-2 above the glass fill line (Samples A through H; Table II, III, Figure 3). The analysis of rust by SEM/EDAX is complicated by the fact that the EDAX spectra for rust and spinel appear the same, e.g. deposits enriched in $\mathrm{Fe}, \mathrm{Ni}$ and $\mathrm{Cr}$. If spinel crystals could not be identified morphologically the phase identification in Table II was given as spinel/rust. Positive identification of $\mathrm{Fe}_{2} \mathrm{O}_{3}$ rust was made in Sample F by XRD analysis (Table III). The bottom of the inner canister closure plug, sample I, 
was not rusted and the identification of traces of rust, halides, and sulfates (Table II) is likely loose particulates from the neck of the canister that were deposited on the closure plug when the canister was lying on its side after sectioning and during sampling.

In almost all cases, when rust was identified as being present, $\mathrm{Ti}, \mathrm{W}, \mathrm{Zn}, \mathrm{Sn}$ or aluminosilicate material from the cutting blade and fluid was also present, indicating that the cutting fluid had penetrated through or around the 11" of glass and come in contact with the halides and sulfates that had condensed on the interior shoulder and neck of the canister. The $\mathrm{Ti}, \mathrm{W}, \mathrm{Zn}$ and $\mathrm{Sn}$ are known to be embedded in the cutting blade of the band-saw used to section the canister, and significant amounts of them are not present in the glass (Table I). The aluminosilicate and rust deposits are, therefore, most likely reaction products of the cutting fluid with the glass and the heat sensitized canister or carbon steel contamination, which occurs on the canister interior during fabrication and handling. Samples of deposits on the glass pour surface, Samples $J$ and $\mathrm{K}$, indicated that a greywhite powder enriched in the W, Ti and $\mathrm{Zn}$ from the cutting blade/fluid was indeed present.

Glass pour surface samples $L$ through $Q$ indicated the same halide, sulfate, borate, rust and/or spinel deposits and $\mathrm{Sn}, \mathrm{Ti}, \mathrm{Zn}$ from the cutting blade/fluid (Table II). Deposits on glass fracture: surfaces from the pour surface to the saw blade surface ( $\mathrm{L}$ through $\mathrm{ZZ}$ ) indicated the same types of deposits (Table II). Some of the samples were still wet (Sample T) although the canister had been sectioned several months before sampling.

Samples HH and II which were glass fracture surfaces from near the top of the canister exhibited cubic crystals of $\mathrm{NaCl}$, molten globules of the $\mathrm{NaF}$ and $\mathrm{Na}_{2} \mathrm{SO}_{4}$ phases and dendritic growths of mixed $(\mathrm{Na}, \mathrm{K}) \mathrm{Cl}$ phase (Figure 4-7). Samples TT and UU indicated that the $(\mathrm{Na}, \mathrm{K}) \mathrm{Cl}$ and $(\mathrm{Na}, \mathrm{K}) \mathrm{SO}_{4}$ phases were immiscible in each other as suggested during heat treatment studies of similar species in the off-gas deposits. ${ }^{7-9}$ Sample VV, which appeared as white deposits on a glass stringer near the interior shoulder of the canister also contained large cubic $\mathrm{NaCl}$ crystals. The solid dried samples flushed from the canister shoulder (Table IV) indicated that the $\mathrm{NaCl}$ had recrystallized to a dendritic form after flushing and drying the halide from the canister interior (Figure 8). Spincl crystals were often found associated with the glass pour surface (Figure 9).

\section{CONCLUSIONS}

Mixed $(\mathrm{Na}, \mathrm{K}) \mathrm{Cl},(\mathrm{Na}, \mathrm{K})_{2} \mathrm{SO}_{4},(\mathrm{Na}, \mathrm{K})$ borates, and a $(\mathrm{Na}, \mathrm{K})$ fluoride phase (either $\mathrm{NaF}$ or $\mathrm{Na}_{2} \mathrm{BF}_{4}$ ) were identified as inorganic species on the interior canister walls, neck, and shoulder above the melt pour surface. Similar deposits were found on the glass melt surface and on glass fracture surfaces. Spinel crystals were only found associated with the glass pour surface.

The stoichiometry of these deposits is similar to the phases identified in scale glass melter off-gas systems. The off-gas deposits form by vapor-phase transport and condensation. Vaporization of species from the melt as it enters the canister and condensation on the interior canister shoulder and neck as the glass is poured is the most likely mechanism by which halide and sulfate species could be deposited in these regions of the canister. Reference amounts of the halides and sulfates were found to be retained in the glass and the glass chemistry, including the distribution of the halides and sulfates, was homogeneous. 
Rust was visibly present and identified by both SEM and XRD analysis. In all cases where rust was observed, heavy metals $(\mathrm{Zn}, \mathrm{Ti}, \mathrm{Sn})$ from the cutting blade/fluid were present indicating that the cutting fluid had penetrated through or around the 11 inches of glass to the interior walls of the top of the canister. The Ti, W, $\mathrm{Zn}$ and $\mathrm{Sn}$ are known to be embedded in the cutting blade of the band saw used to section the canister and significant amounts of these species are not present in the glass. Aluminosilicate and rust deposits identified are, therefore, reaction products of the cutting fluid with the glass and with the heat sensitized canister or with carbon steel contamination on the canister interior which occurs during fabrication and handling. Measured relative humidity in sealed simulated waste glass canisters indicates that only minimal water vapor is present so that internal corrosion of the canister, as observed in the SGM 10-2 sectioned canister, will not occur.

\section{ACKNOWLEDGEMENT}

Special thanks are due to W. G. Ramsey of Clemson University and John R. Harbour of the Glass Technology Group of the Savannah River Technology Center for their assistance in sampling of the canister glass. 


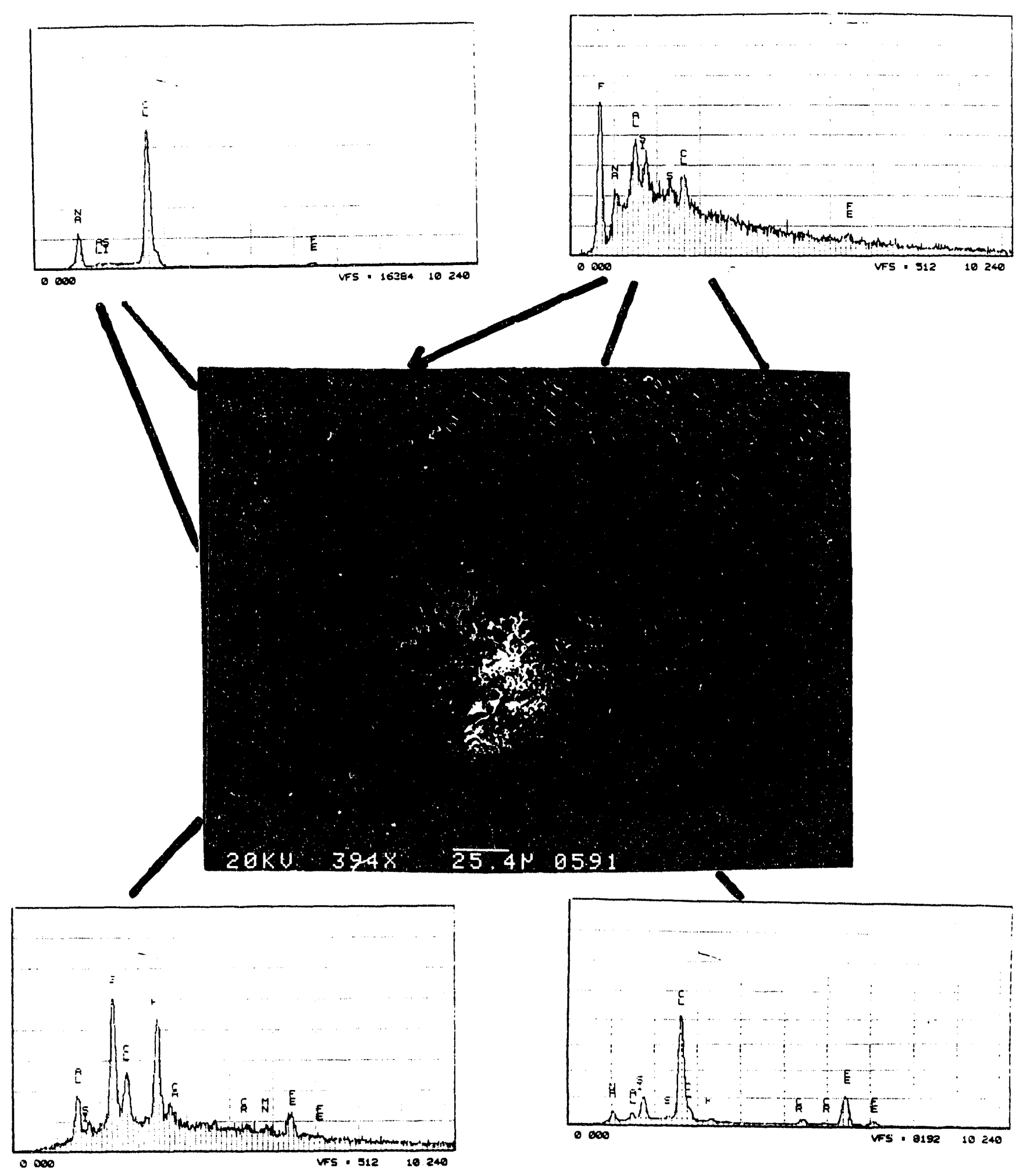

Figure 4. Morphology of alkali halide and sulfate species in canister SGM 10-2 glass fracture surface (Sample HH). 

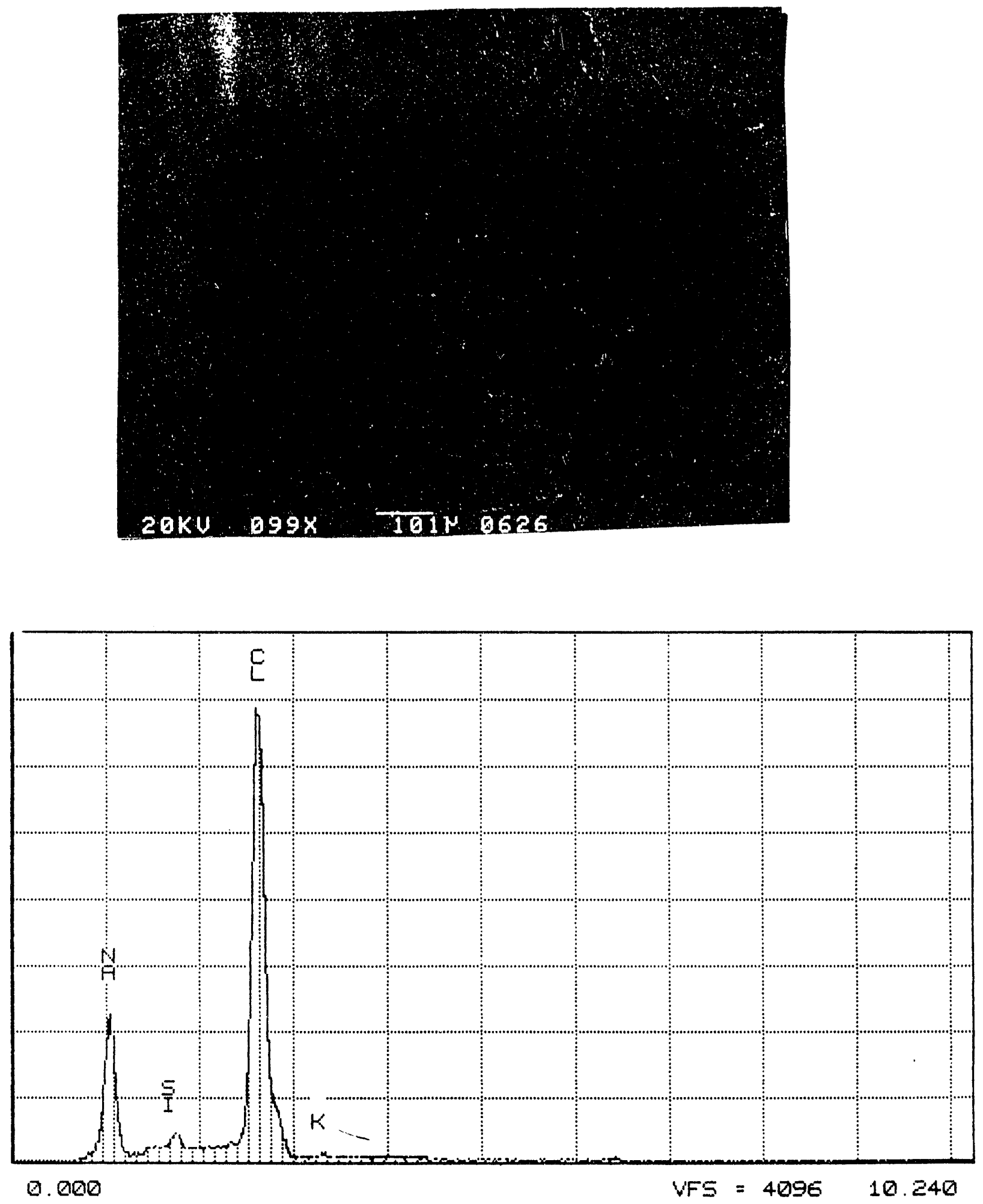

Figure 5. Morphology of molten alkali halide (dark streaks) in canister SGM 10-2 glass fracture surface (Sample II). 

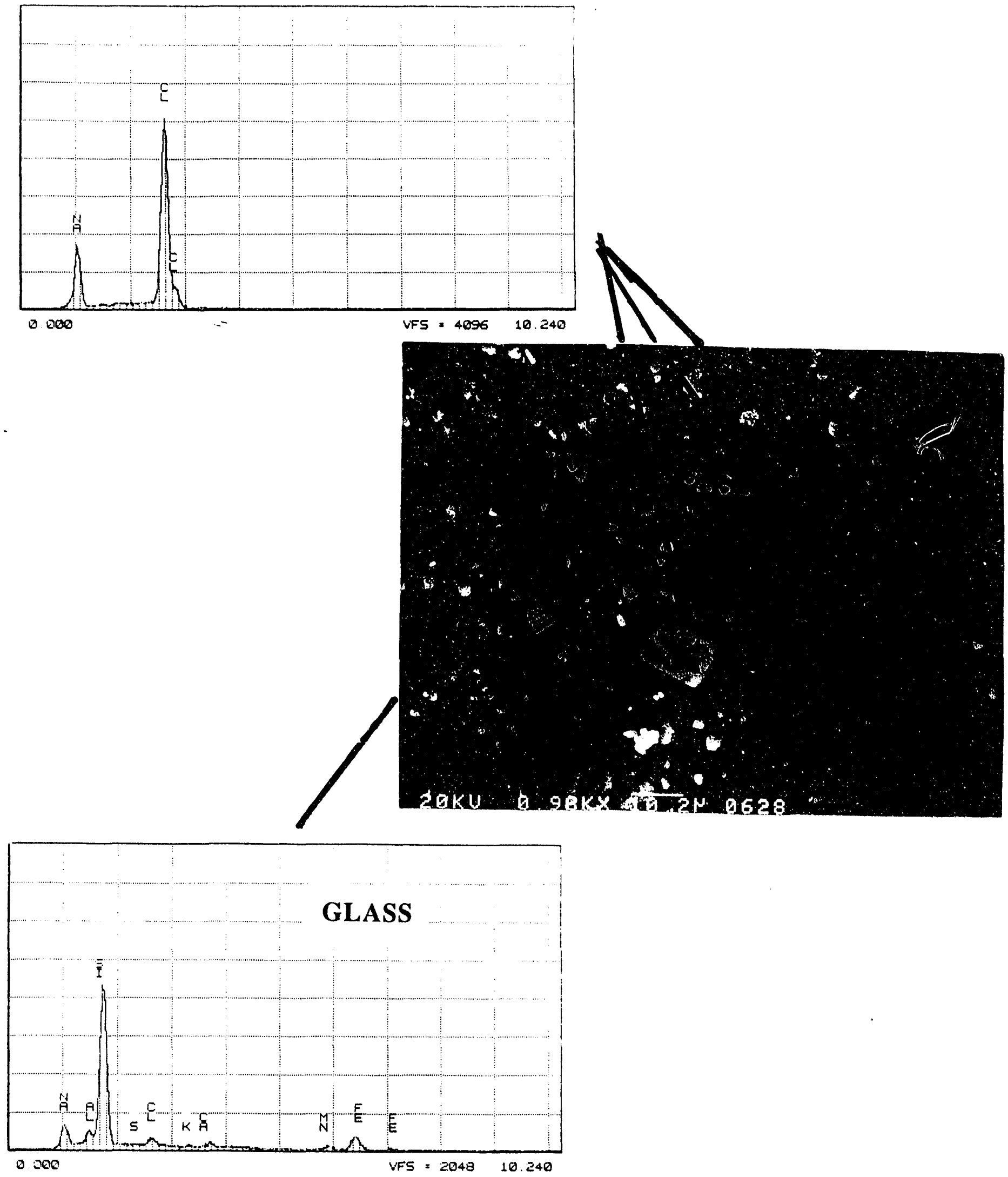

Figure 6. Different morphologies of alkali halides in canister SGM 10-2 glass fracture surface (Sample II). 

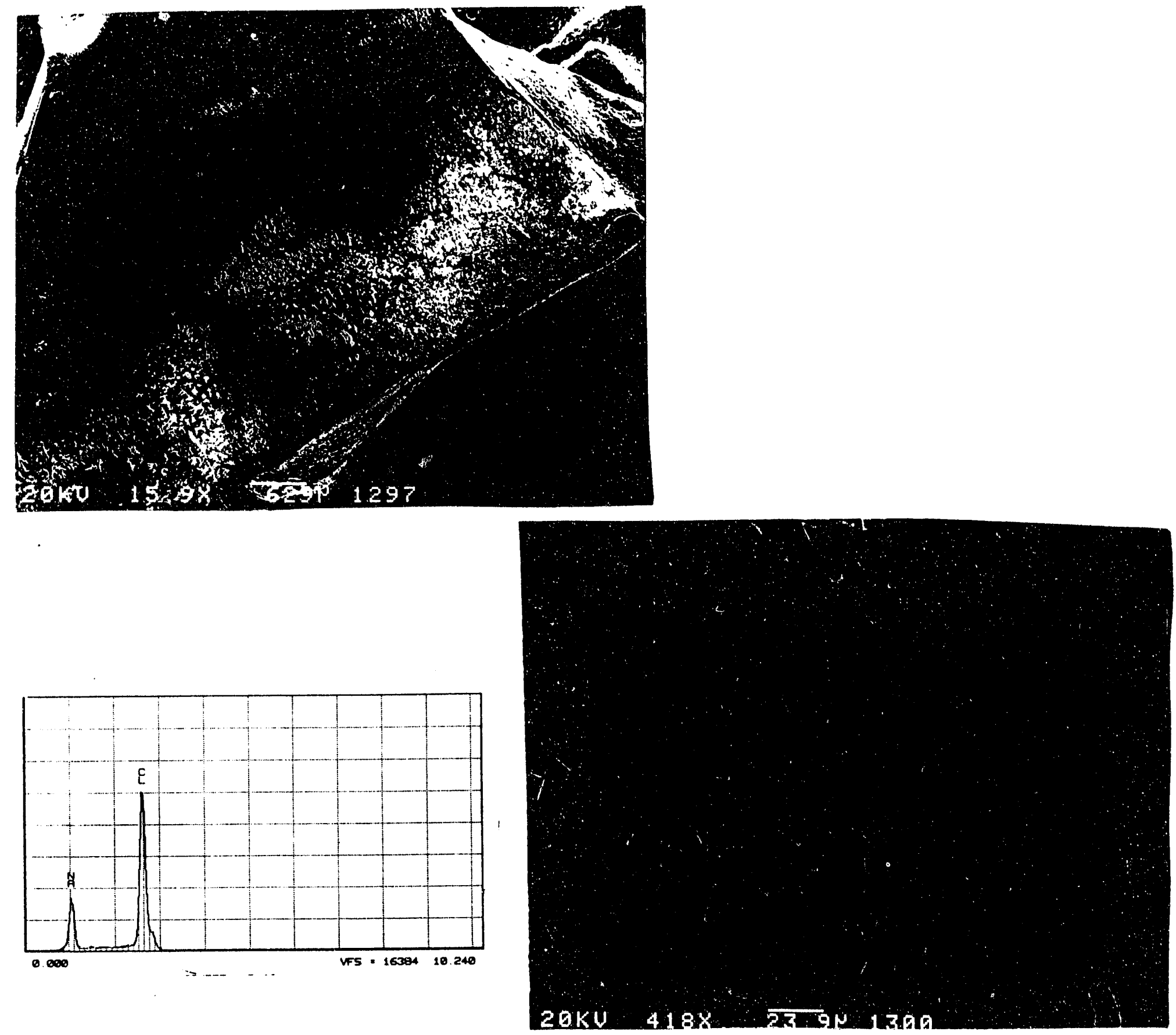

Figure 7. White alkali halide deposits in canister SGM 10-2 glass stringer near interior shoulder of canister (Sample VV). Very large cubic $\mathrm{NaCl}$ crystals visible at higher magnification. 

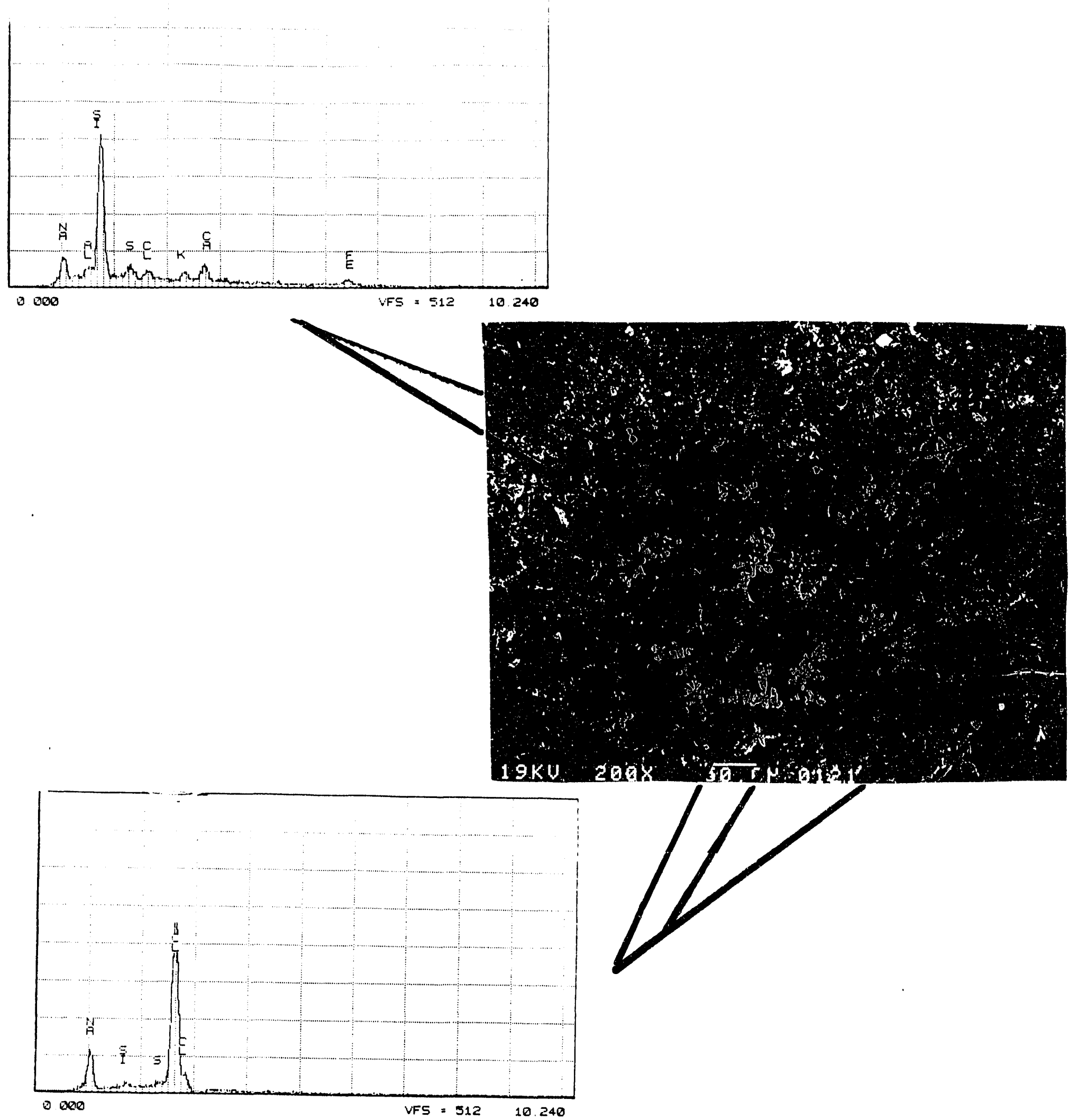

Figure 8. Dendritic alkali chloride and globular mixed chloride and sulfate deposits in canister SGM 10-2 that were flushed from the canister shoulder and sides with deionized water and dried at $90^{\circ} \mathrm{C}$ for phase identification (Sample Flush Top Solid). 


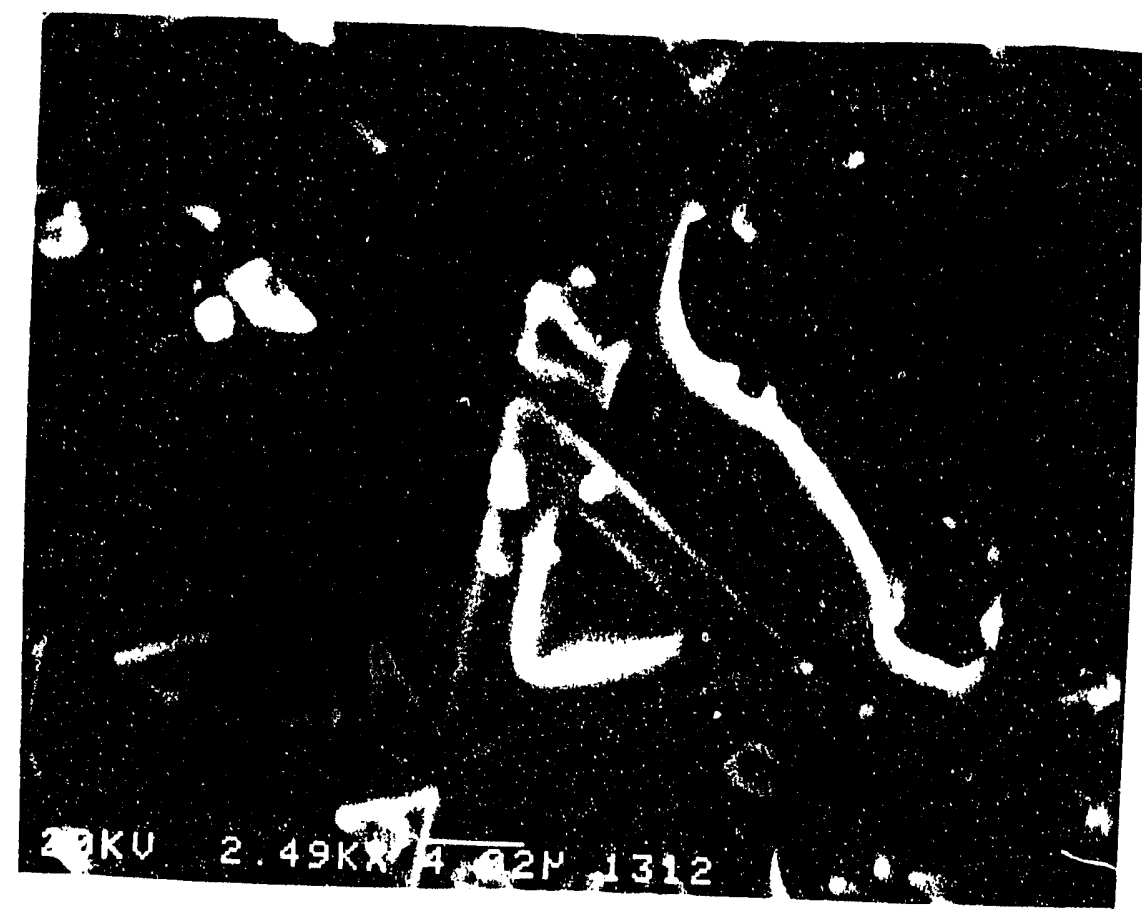
Figure 9. Morphology of isometric spinel crystals associated with the melt pour surface in
Canister SGM 10-2 (Sample ZZ). 
This page intentionally left blank.

$-34-$ 


\section{REFERENCES}

1. Office of Civilian Radioactive Waste Management, Waste Acceptance Preliminary Specifications for Vitrified High-Level Waste Forms, DRAFT, June 1991.

2. Waste Form Compliance Plan for the Defense Waste Processing Facility, Revision 0, USDOE Document WSRC-SW4-6, Westinghouse Savannah River Company, Aiken, SC, March 1990.

3. R.G. Baxter, "Defense Waste Processing Facility Wasteform and Canister Description," USDOE Report DP-1606, Rev. 2, E.I. duPont deNemours \& Co. Savannah River Plant, Aiken, SC (December, 1988).

4. J.R. Fowler, "Projected Chemical Compositions of SRP Waste Glass for the Waste Acceptance Preliminary Specifications," USDOE Report DPST-87-713, E.I. duPont deNemours \& Co. Savannah River Plant, Aiken, SC (March, 1988).

5. J.R. Fowler and M.J. Plodinec, "Projected Compositions and Radiogenic Properties of DWPF Glasses," Proceedings of the Third International Conference on High Level Radioactive Waste Management, Vol. 1, 904-910 (1992).

6. D.F. Bickford, A.A. Ramsey, C.M. Jantzen, and K.G. Brown, "Control of Radioactive Waste Glass Melters: I. Preliminary General Limits at Savannah River,"J. Am. Ceram. Soc., 73 [10], 2896-2902 (1990).

7. C.M. Jantzen, “Glass Melter Off-gas System Pluggages: Cause, Significance, and Remediation," USDOE Report WSRC-TR-90-205, Rev. 0, Westinghouse Savannah River Co., Savannah River Site, Aiken, SC (March, 1991).

8. C.M. Jantzen, “Glass Melter Off-gas System Pluggages: Cause, Significance, and Remediation," Proceedings of the 5th International Symposium on Ceramics in Nuclear Waste Management, G.G. Wicks, D.F. Bickford, and R. Bunnell (Eds.), American Ceramic Society, Westerville, OH, 621-630 (1991).

9. C.M. Jantzen, A.S. Choi, and C.T. Randall, "Remediation of Off-Gas System Deposits in a Radioactive Waste Glass Melter," High Level Radioactive Waste and Spent Fuel Management, Vol. 2, S.C. Slate, L.C. Oyen, K.J. Lee, and W.Z. Oh (Eds.), Korean Nuclear Society, Seoul, Korea, 21-32, (1991).

10. J.W. Kelker, "Development of the DWPF Canister Temporary Shrink-Fit Seal," USDOE Report DP-1720, E.I. duPont deNemours \& Co., Savannah River Laboratory, Aiken, SC (1986).

11. J.R. Harbour, T.J. Miller, and M.J. Whitaker, "The Physical Properties and Chemical Composition of the Gas Within the Free Volume of Canistered Waste Forms," USDOE Report WSRC-TR-90-521, Westinghouse Savannah River Co., Savannah River Site, Aiken, SC (November, 1990). 
12. J.R. Harbour, "Qualification of Data by C.A. Langton on Internal Canister Environment," USDOE Report WSRC-RP-90-1199, Westinghouse Savannah River Co., Savannah River Site, Aiken, SC (October, 1990).

13. C.A. Langton, "DWPF Canister Corrosion Studies Literature Review and Recommendations," USDOE Report DPST-82-978, E.I. duPont deNemours \& Co., Savannah River Laboratory, Aiken, SC (1982).

14. M.E. Smith, M.R. Baron, S.L. Goudelock, G.F. Rabon, A.M. Wehner, "DWPF Scale Glass Melter: Run Plan for SGM-10," USDOE Report DPST-88-355, E.I. duPont deNemours \& Co., Savannah River Laboratory, Aiken, SC (1988).

15. J.R. Harbour and C.M. Jantzen, "R/D Task Plan: Organic and Inorganic Analysis of Volatilized and Condensed Species Within SRL Canistered Waste Forms," USDOE Report WSRC-RP-90-179, Westinghouse Savannah River Co., Savannah River Laboratory, Aiken, SC (1990).

16. A.S. Choi, "Freeze and Restart of the DWPF Scale Glass Melter," USDOE Report WSRC-RP-89-634, Westinghouse Savannah River Co., Savannah River Laboratory, Aiken, SC (1989).

17. A.S. Choi, M.E. Smith, K.R. Crow, M.R. Baron, G.F. Rabon, S.L. Goudelock, A.M. Wehner, "Summary of Campaigns SGM-9 and SGM-10 of the DWPF Scale Glass Melter," USDOE Report DPST-88-626, E.I. duPont deNemours \& Co., Savannah River Laboratory, Aiken, SC (1988). 


\section{APPENDIX I \\ DETAILS OF THE GLASS CHEMICAL ANALYSES}


GLASS 10=2/3 + O EDGE - A (ADS 200038840$)$

\begin{tabular}{|c|c|c|c|c|c|c|c|c|c|c|c|}
\hline & $\begin{array}{l}\text { ELEMENT } \\
\text { WT\% }\end{array}$ & $\begin{array}{l}\text { GRAV } \\
\text { FACTOR }\end{array}$ & $\begin{array}{l}\text { OXIDE } \\
\text { WT\% }\end{array}$ & $\begin{array}{l}\text { NOFM OXIDE } \\
\text { WT\% }\end{array}$ & M.W. & $\begin{array}{c}\text { MOLE } \\
\text { FAACTION }\end{array}$ & $\begin{array}{l}\text { NORMOXIDE } \\
\text { MOLE \% }\end{array}$ & $\begin{array}{l}\text { COMPONENT } \\
\text { FREE ENERGYY }\end{array}$ & $\begin{array}{l}\text { PREEGERGY } \\
\text { HYDFATION }\end{array}$ & $\mathrm{NBO}$ & KCAL'KG \\
\hline Al2O3 & 1.9120 & 1.8895 & 3.6127 & 3.6342 & 101.9600 & 0.0354 & 2.2931 & 3.0400 & $\quad 0.0697$ & -0.0459 & 1.0772 \\
\hline$\infty$ & 0.8920 & 1.3992 & 1.2481 & 1.2555 & 56.0794 & 0.0223 & 1.4403 & -16.1160 & -0.2321 & 0.0288 & -3.5867 \\
\hline $\mathrm{Fe2O3}$ & 7.6060 & 1.4297 & 10.8743 & 10.9391 & 159.6922 & 0.0681 & 4.4069 & 15.5000 & 0.6831 & .0 .0881 & 10.5548 \\
\hline FoO & N/A & 1.2865 & 0.0000 & 0.0000 & 71.8464 & 0.0000 & 0.0000 & -14.6090 & 0.0000 & 0.0000 & $0.000 n$ \\
\hline 100 & 0.4950 & 1.6583 & 0.8209 & 0.8257 & 40.3114 & 0.0204 & 1.3178 & -13.8880 & -0.1830 & 0.0264 & -2.8280 \\
\hline Mo & 1.6640 & 1.2912 & 2.1486 & 2.1614 & 70.9374 & 0.0303 & 1.9601 & .14 .8710 & -0.2915 & 0.0392 & -4.5041 \\
\hline $\mathrm{N}=20$ & 9.1440 & 1.3480 & 12.3261 & 12.3995 & 61.9790 & 0.1989 & 12.8705 & -28.8150 & -3.7086 & 0.2574 & -57.3060 \\
\hline $\mathrm{Li2O}$ & 2.0300 & 2.1525 & 4.3696 & 4.3956 & 28.8774 & 0.1463 & 9.4648 & -22.7400 & -2.1523 & 0.1893 & -33.2573 \\
\hline No & 0.7290 & 1.2726 & 0.9277 & 0.9333 & 74.7094 & 0.0124 & 0.8036 & .14 .3470 & -0.1153 & 0.0161 & -1.7816 \\
\hline $\mathrm{SiO} 2$ & 23.2530 & 2.1393 & 49.7451 & 50.0414 & 60.0848 & 0.8279 & 53.5796 & $\mathbf{5 . 5 9 0 0}$ & 1.3855 & 0.0000 & 21.5633 \\
\hline $\mathrm{Cr} 2 \mathrm{O3}$ & 0.0810 & 1.4616 & 0.1184 & 0.1191 & 151.9974 & 0.0008 & 0.0504 & 37.3600 & 0.0188 & -0.0010 & 02910 \\
\hline 8203 & 3.6360 & 3.2201 & 11.7083 & 11.7780 & 69.6204 & 0.1682 & 10.8835 & -9.9300 & -1.0807 & 0.0000 & -16.6996 \\
\hline we & & 1.1344 & 0.0000 & 0.0000 & 270.0388 & 0.0000 & 0.0000 & -6.8000 & 0.0000 & 0.0000 & 0.0000 \\
\hline Thoe & & 1.1379 & 0.0000 & 0.0000 & 264.0400 & 0.0000 & 0.0000 & -2.5330 & 0.0000 & 0.0000 & 0.0000 \\
\hline sro & 0.0380 & 1.1826 & 0.0449 & 0.0452 & 103.6194 & 0.0004 & 0.0281 & -24.4000 & -0.0068 & 0.0006 & $-0.105 \mathrm{C}$ \\
\hline 2002 & 0.4930 & 1.3508 & 0.6659 & 0.6689 & 123.2188 & 0.0054 & 0.3498 & 45.1000 & 0.1577 & 0.0140 & 2.4375 \\
\hline TiO2 & 0.0910 & 1.6680 & 0.1518 & 0.1527 & 79.8988 & 0.0019 & 0.1229 & 15.9900 & 0.0197 & 0.0049 & 0.3038 \\
\hline 120 & 0.3340 & 1.2046 & 0.4023 & 0.4047 & 94.2034 & 0.0043 & 0.2764 & -41.7350 & -0.1154 & 0.0055 & -1.7825 \\
\hline $0<0$ & & 1.0602 & 0.0000 & 0.0000 & 281.8094 & 0.0000 & 0.0000 & -46.8200 & 0.0000 & 0.0000 & 0.0000 \\
\hline $\mathrm{Sb}_{203}$ & & 1.1970 & 0.0000 & 0.0000 & 291.4982 & 0.0000 & 0.0000 & 0.0000 & 0.0000 & 0.0000 & 0.0000 \\
\hline P2OS & 0.0460 & 2.2910 & 0.1054 & 0.1060 & 141.9370 & 0.0007 & 0.0481 & -53.6200 & -0.0258 & 0.0000 & -0.3881 \\
\hline $\mathrm{N}+2 \mathrm{O} 3$ & & 1.1660 & 0.0000 & 0.0000 & 336.4782 & 0.0000 & 0.0000 & .28 .3200 & 0.0000 & 0.0000 & 0.0000 \\
\hline $\mathrm{LeOO}$ & & 1.1728 & 0.0000 & 0.0000 & 325.8100 & 0.0000 & 0.0000 & -31.1400 & 0.0000 & 0.0000 & 0.0000 \\
\hline roos & & 1.2699 & 0.0000 & 0.0000 & 225.8082 & 0.0000 & 0.0000 & -12.9500 & 0.0000 & 0.0000 & 0.0000 \\
\hline Bo & 0.0130 & 1.1165 & 0.0145 & 0.0146 & 153.3394 & 0.0001 & 0.0061 & -30.5700 & -0.0019 & 0.0001 & -0.0289 \\
\hline$+\infty$ & & 1.0772 & 0.0000 & 0.0000 & 223.1900 & 0.0000 & 0.0000 & -8.8100 & 0.0000 & 0.0000 & 0.0000 \\
\hline $\operatorname{coc} 0$ & & 1.2284 & 0.0000 & 0.0000 & 172.1200 & 0.0000 & 0.0000 & 11.3450 & 0.0000 & 0.0000 & 0.0000 \\
\hline $\mathrm{MOOS3}$ & & 1.5003 & 0.0000 & 0.0000 & 143.9382 & 0.0000 & 02000 & -21.7500 & 0.0000 & 0.0000 & 0.0000 \\
\hline Zno & 0.098 & 1.2447 & 0.1232 & 0.1240 & 81.3800 & 0.0015 & 0.0980 & -2.4000 & -0.0024 & 0.0020 & -0.0363 \\
\hline a) & & 1.2518 & 0.0000 & 0.0000 & 79.5454 & 0.0000 & 0.0000 & 1.7900 & 0.0000 & 0.0000 & 0.0000 \\
\hline $\mathrm{F} \bullet 2+1 \mathrm{~F} \bullet 3+$ & 0 & & & & & & & & & & \\
\hline Cl & 0.3971 & & 0.3971 & 0.3971 & & & & & & & \\
\hline $\mathbf{F}$ & 0.0253 & & 0.0253 & 0.0253 & & & & & & & \\
\hline sou & 0.1633 & & 0.1633 & 0.1633 & & & & & & & \\
\hline \multirow[t]{2}{*}{ OXDE SUMS } & 52.5560 & & 99.4079 & 100.0000 & & 1.5452 & 100.0000 & & .5 .5713 & 0.4492 & -86.0876 \\
\hline & & & \multirow[b]{2}{*}{ MOLAR SUMS } & & NORMAUZED & & & \multirow{2}{*}{$\begin{array}{c}\text { SUM + pH Si } \\
\text { SUM }+p H B \\
p H=\end{array}$} & -5.5713 & & \\
\hline \multicolumn{2}{|c|}{ MOLAR BALANCE (M) } & & & MOLE\% & MOLE \% & & & & -5.5713 & & \\
\hline $\mathrm{K} 2 \mathrm{SO}_{4}$ & 0.2962 & & SALTS & 1.1727 & 0.9537 & & & & & & \\
\hline $\mathrm{KCl}$ & 0.8345 & & BORATES & 22.1288 & 17.9965 & & & & & & \\
\hline $\mathrm{KBF} 4$ & 0.0419 & & CHROMATES & 0.2523 & 0.2052 & & & & & & \\
\hline NeBOe & 22.1288 & & OXIDES & 99.4079 & 80.8446 & & & & & & \\
\hline \multirow[t]{2}{*}{$\mathrm{Na}_{2} \mathrm{CrO} 4$} & 0.2523 & & & & & & & & & & \\
\hline & & & TOTAL & 122.9616 & 100.0000 & & & & & & \\
\hline K excene(M) & .0 .01 & & & & & & & & & & \\
\hline Nu excese (M) & 0.06 & & & & & & & & & & \\
\hline 804 excees (M) & 0.00 & & & & & & & & & & \\
\hline Cl excess & 0.00 & & & & & & & & & & \\
\hline F excosen & 0.00 & & & & & & & & & & \\
\hline D exceses & 0.00 & & & & & & & & & & \\
\hline ALKSSIO2 = & & 0.422 & & TEST= & & & & & & & \\
\hline ALK+ B2O3/SIO2 & & 0.625 & & TIME $=$ & & & & & & & \\
\hline B203/S102 = & & 0.203 & & & & & & & & & \\
\hline VISCOSITY QTE & $P\left({ }^{\circ} \mathrm{C}\right)$ & 34.482 & & $S A / V=$ & & & & & & & \\
\hline LOUIDUS RATIO & & 0.079 & & & & & & & & & \\
\hline UOUIDUS $\left({ }^{\circ} \mathrm{C}\right)=$ & 4" & 0.7677135 & 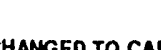 & & & & & & & & \\
\hline
\end{tabular}

(SPAEAdSMEet ReVISION 5.0, OCTOBer 23, 1990)

MPOXIDE COMPONENT FEEEERGY NDO KCAL'KG

NOTE LUUMDUS AND VISCOSTTY FORMULAS CHANGED TO CALCULATE ALL. FE AS FE203 (OCTOBER 23, 1900) 
GLASS ID=2/3 + O EDGE - B (ADS $\$ 200038841)$

\begin{tabular}{|c|c|c|c|c|c|c|c|c|c|c|c|}
\hline & $\begin{array}{c}\text { EQNENT } \\
\text { WT\% }\end{array}$ & $\begin{array}{l}\text { GRAV } \\
\text { FACTOR }\end{array}$ & $\begin{array}{l}\text { OXIDE } \\
\text { WT\% }\end{array}$ & $\begin{array}{c}\text { NOAMONDE } \\
\text { WT\% }\end{array}$ & M.W. & $\begin{array}{c}\text { MOLE } \\
\text { FRACTION }\end{array}$ & $\begin{array}{l}\text { NOPMOXDE } \\
\text { MOLE \% }\end{array}$ & $\begin{array}{l}\text { COMPONENT } \\
\text { FREEENERGY }\end{array}$ & $\begin{array}{l}\text { FREEQNEPGY } \\
\text { HYDRATION }\end{array}$ & NBO & KCAL/KG \\
\hline $\mathrm{Al}_{2} \mathrm{O}_{3}$ & 1.4550 & 1.8895 & 2.7492 & 3.1276 & 101.9600 & 0.0270 & 1.9597 & 3.0400 & 0.0596 & -0.0392 & 0.8197 \\
\hline$\infty$ & 0.7650 & 1.3992 & 1.0704 & 1.2177 & 56.0794 & C.0191 & 1.3873 & .18 .1160 & -0.2236 & 0.0277 & -3.0761 \\
\hline$F \bullet 203$ & 6.2390 & 1.4297 & 8.9199 & 10.1476 & 159.6922 & 0.0559 & 4.0597 & 15.5000 & 0.6293 & -0.0812 & B. 6578 \\
\hline Fo & N/A & 1.2865 & 0.0000 & 0.0000 & 71.8464 & 0.0000 & 0.0000 & .14 .6090 & 0.0000 & 0.0000 & 0.0000 \\
\hline$M \infty O$ & 04240 & 1.6583 & 0.7031 & 0.7999 & 40.3114 & 0.0174 & 1.2677 & .13 .8880 & .0 .1761 & 0.0254 & -2.4224 \\
\hline Aro & 1.4370 & 1.2912 & 1.8555 & 2.1108 & 70.9374 & 0.0262 & 1.9011 & -14.8710 & -0.2827 & 0.0380 & -3.8887 \\
\hline$N=00$ & 8.7680 & 1.3480 & . 993 & 13.4460 & 61.9790 & 0.1907 & 13.8600 & -28.8150 & -3.9938 & 0.2772 & -54.8496 \\
\hline Li2n & 1.8100 & 2.1525 & 3.8960 & 4.4322 & 29.8774 & 0.1304 & 0.4776 & -22.7400 & -2.1552 & 0.1896 & .296531 \\
\hline No & 0.5830 & 1.2726 & 0.7418 & 0.6440 & 74.7094 & 0.0099 & 0.7218 & -14.3470 & -0.1036 & 0.0144 & .14248 \\
\hline $\mathrm{SiO} 2$ & 20.6360 & 2.1393 & 44.1466 & 50.2226 & 60.0848 & 0.7347 & 53.4012 & 5.5900 & 1.3382 & 0.0000 & 184120 \\
\hline cr203 & 0.0790 & 1.4616 & 0.1155 & 0.1314 & 151.9974 & 0.0008 & 0.0552 & 37.3600 & 0.0206 & -0.0011 & 0.2838 \\
\hline 8203 & 3.2450 & 3.2201 & 10.4492 & 11.8674 & 69.62 .04 & 0.1501 & 10.9085 & -9.9300 & -1.0832 & 0.0000 & .14 .0038 \\
\hline 102 & & 1.1344 & 0.0000 & 0.0000 & 270.0388 & 0.0000 & 0.0000 & -6.8000 & 0.0000 & 0.0000 & 0.0000 \\
\hline Thor & & 1.1379 & 0.0000 & 0.0000 & 264.0400 & 0.0000 & 0.0000 & -2.5330 & 0.0000 & 0.0000 & 0.0000 \\
\hline so & 0.0330 & 1.1826 & 0.0390 & 0.0444 & 103.6194 & 0.0004 & 0.0274 & $-24,4000$ & -0.0067 & 0.0005 & $\cdot 0.0919$ \\
\hline 2102 & 0.1790 & 1.3508 & 0.6470 & 0.7361 & 123.2188 & 0.0053 & 0.3817 & 45.1000 & 0.1721 & 0.0153 & 2.3682 \\
\hline TiO2 & 0.0780 & 1.6680 & 0.1301 & 0.1480 & 70.8988 & 0.0016 & 0.1184 & 15.9900 & 0.0188 & 0.0047 & 0.2604 \\
\hline 120 & 0.3360 & 1.2046 & 0.4047 & 0.4605 & 94.2034 & 0.0043 & 0.3123 & -41.7350 & -0.1303 & 0.0062 & -1.7931 \\
\hline$c=0$ & & 1.0602 & 0.0000 & 0.0000 & 281.8094 & 0.0000 & 0.0000 & -46.8200 & 0.0000 & 0.0000 & 0.0000 \\
\hline 80203 & & 1.1870 & 0.0000 & 0.0000 & 291.4982 & 0.0000 & 0.0000 & 0.0000 & 0.0000 & 0.0000 & 0.0000 \\
\hline PCOS & 0.0300 & 2.2910 & 0.0687 & 0.0782 & 141.8370 & 0.0005 & 0.0352 & -53.6200 & -0.0189 & 0.0000 & -0.2596 \\
\hline$N+200$ & & 1.1660 & 0.0000 & 0.0000 & 336.4782 & 0.0000 & 0.0000 & -28.3200 & 0.0000 & 0.0000 & 0.0000 \\
\hline Le203 & & 1.1728 & 0.0000 & 0.0000 & 325.8100 & 0.0000 & 0.0000 & -31.1400 & 0.0000 & 0.0000 & 00000 \\
\hline Y2O3 & & 1.2699 & 0.0000 & 0.0000 & 225.8082 & 0.0000 & 0.0000 & -12.9500 & 0.0000 & 0.0000 & 00000 \\
\hline$B \times$ & 0.0100 & 1.1165 & 0.0112 & 0.0127 & 153.3394 & 0.0001 & 0.0053 & -30.5700 & -0.0016 & 0.0001 & .00223 \\
\hline$P \infty O$ & & 1.0772 & 0.0000 & 0.0000 & 223.1000 & 0.0000 & 0.0000 & .8 .8100 & 0.0000 & 0.0000 & 0.0000 \\
\hline con & & 1.2284 & 0.0000 & 0.0000 & 172.1200 & 0.0000 & 0.0000 & $\$ 1.3450$ & 0.0000 & 0.00010 & 0.0000 \\
\hline$M 000$ & & 1.5003 & 0.01100 & 0.0000 & 143.9382 & 0.0000 & 0.0000 & -21.7500 & 0.0000 & 0.00010 & 0.0000 \\
\hline $2 n o$ & 0.108 & 1.2447 & 0.1344 & 0.1529 & 81.3800 & 0.0017 & 0.1201 & -2.4000 & .0 .0029 & 0.002. & -0.0396 \\
\hline 0 & & 1.2518 & 0.0000 & 0.0000 & 79.5454 & 0.0000 & 0.0000 & 1.7900 & 0.0000 & 0.00013 & 0.0000 \\
\hline $\mathrm{F} \cdot 2+\mathrm{F} 03+$ & 0 & & & & & & & & & & \\
\hline Cl & 0.5004 & & 0.5004 & 0.5004 & & & & & & & \\
\hline $\mathbf{F}$ & 0.0328 & & 0.0328 & 0.0328 & & & & & & & \\
\hline son & 0.1838 & & 0.1838 & 0.1838 & & & & & & & \\
\hline \multirow[t]{2}{*}{ ONOE SUMS } & 46.5150 & & 87.9018 & 100.0000 & & 1.3759 & 100.0000 & & -5.9397 & 0.480 & .81 .7240 \\
\hline & & & & & NORMALIEEO & & & SUM + pH Si & -5.9397 & & \\
\hline \multicolumn{2}{|c|}{ molar baLaNCE (M) } & & MOLAR SUMB & MOLE & MOLE\% & & & $\mathrm{SUM}+\mathrm{pHB}$ & .5 .9397 & & \\
\hline KasO4 & 0.3334 & & SALTS & 1.4394 & 1.3165 & & & $\mathrm{PH}=$ & & & \\
\hline $\mathrm{KCl}$ & 1.0516 & & BOAATES & 18.7492 & 18.0628 & & & & & & \\
\hline KBF4 & 0.0543 & & CPOMATES & 0.2460 & 0.2250 & & & & & & \\
\hline Nener & 19.7492 & & OXIDES & 87.9018 & 80.3957 & & & & & & \\
\hline \multirow[t]{2}{*}{ Necros } & 0.2460 & & & & & & & & & & \\
\hline & & & TOTAL & 109.3364 & 100.0000 & & & & & & \\
\hline K exceas(M) & .0 .01 & & & & & & & & & & \\
\hline Na oxecess (M) & 0.08 & & & & & & & & & & \\
\hline son excess in & 0.00 & & & & & & & & & & \\
\hline Cl oxceses & 0.00 & & & & & & & & & & \\
\hline F exceses & 0.00 & & & & & & & & & ' & \\
\hline B exceses & 0.00 & & & & & & & & & & \\
\hline ALKSIO2 = & & 0.443 & & TEST: & & & & & & & \\
\hline ALK+ B203sion & & 0.647 & & TIME $=$ & & & & & & & \\
\hline B2038102 = & & 0.204 & & & & & & & & & \\
\hline VBCOSTY OTE & $A P(\cdot C)$ & 29.358 & & SA/V: & & & & & & & \\
\hline $\begin{array}{l}\text { UOLnOUS RATL } \\
\text { UOLOUS }\left({ }^{\circ} \mathrm{C}\right)=\end{array}$ & & 0.072 & & & & & & & & & \\
\hline LOUNDUS $\left({ }^{\circ} \mathrm{C}\right)=$ & Mrancung & 7.3222662 & & & & & & & & & \\
\hline
\end{tabular}

(SPRSADSHEET REVISION 5.0, OCTOBER 23, 1990) 
GLASS ID $=2 / 3$ + O CENTER - A (ADS 200038042)

\begin{tabular}{|c|c|c|c|c|c|c|c|c|c|c|c|}
\hline & $\begin{array}{c}\text { EAMENT } \\
W T \%\end{array}$ & $\begin{array}{l}\text { GRAV } \\
\text { FACTOR }\end{array}$ & $\begin{array}{l}\text { OXIOE } \\
W T \%\end{array}$ & $\begin{array}{c}\text { NOPMOXXEE } \\
\text { WT\% }\end{array}$ & M.W. & $\begin{array}{c}\text { MOLE } \\
\text { FRACTION }\end{array}$ & $\begin{array}{l}\text { NOPMOXIDE } \\
\text { MOLE } \%\end{array}$ & $\begin{array}{l}\text { COMPONENT } \\
\text { FREE ENERGY }\end{array}$ & $\begin{array}{l}\text { FREE ENEACY } \\
\text { HYDRATION }\end{array}$ & NBO & KCAL/KG \\
\hline $\mathrm{A} 2 \mathrm{O} 3$ & 1.7630 & 1.8895 & 3.3312 & 3.5450 & 101.9600 & 0.0327 & 2.2373 & 3.0400 & 0.0680 & -0.0447 & 0.8932 \\
\hline$\infty$ & 0.8930 & 1.3992 & 1.2495 & 1.3287 & 56.0794 & 0.0223 & 1.5257 & -16.1160 & -0.2458 & 0.0305 & -3.5907 \\
\hline$F 0203$ & 7.3280 & 1.4297 & 10.4768 & 11.1493 & 159.6922 & 0.0856 & 4.4926 & 15.5000 & 0.6963 & .0 .0899 & 10.1690 \\
\hline$F \infty$ & N/A & 1.2865 & 0.0000 & 0.0000 & 71.8464 & 0.0000 & 0.0000 & -14.6090 & 0.0000 & 0.0000 & 0.0000 \\
\hline $1+0$ & 0.4780 & 1.6583 & 0.7927 & 0.8435 & 40.3114 & 0.0197 & 1.3465 & -13.8880 & -0.1870 & 0.0269 & -2.7309 \\
\hline mo & 1.5960 & 1.2912 & 2.0608 & $2 .: 890$ & 70.9374 & 0.0291 & 1.9893 & -14.8710 & -0.2958 & 0.0398 & .4 .3201 \\
\hline$N=20$ & 7.6330 & 1.3480 & 10.2893 & 10.9497 & 61.9790 & 0.1660 & 11.3681 & -28.8150 & -3.2757 & 0.2274 & -47.8365 \\
\hline Li2O & 1.9530 & 2.1525 & 4.2038 & 4.4737 & 29.8774 & 0.1407 & 9.6350 & -22.7400 & -2.1910 & 0.1927 & -31.8958 \\
\hline NO & 0.6970 & 1.2726 & 0.8870 & 0.9438 & 74.7094 & 0.0118 & 0.8130 & -14.3470 & -0.1166 & 0.0163 & -1.7034 \\
\hline $\mathrm{SOO} 2$ & 22.3300 & 2.1393 & 47.7706 & 50.8368 & 60.0848 & 0.7951 & 54.4431 & 5.5900 & 1.5090 & 0.0000 & 22.0360 \\
\hline craos & 0.0940 & 1.4616 & 0.1374 & 0.1462 & 151.9074 & 0.0000 & 0.0619 & 37.3600 & $0.02 \% 1$ & -0.0012 & 0.3377 \\
\hline 8203 & 3.5210 & 3.2201 & 11.3380 & 12.0657 & 69.6204 & 0.1629 & 11.1518 & -9.9300 & -1.1074 & 0.0000 & -16.1714 \\
\hline we & & 1.1344 & 0.0000 & 0.0000 & 270.0388 & 0.0000 & 0.0000 & .6 .8000 & 0.0000 & 0.0000 & 0.0000 \\
\hline mos & & 1.1379 & 0.0000 & 0.0000 & 264.0400 & 0.0000 & 0.0000 & -2.5330 & 0.0000 & 0.0000 & 0.0000 \\
\hline sro & 0.0340 & 1.1826 & 0.0402 & 0.0428 & 103.6104 & 0.0004 & 0.0266 & .24 .4000 & -0.0065 & 0.0005 & -0.0947 \\
\hline 202 & 0.4700 & 1.3508 & 0.6349 & 0.6756 & 123.2188 & 0.0052 & 0.3528 & 45.1000 & 0.1591 & 0.0441 & 2.3237 \\
\hline TiO2 & 0.0840 & 1.6680 & 0.1401 & 0.1491 & 79.8988 & 0.0018 & 0.1201 & 15.9900 & 0.0192 & 0.0048 & 0.2804 \\
\hline 100 & 0.3320 & 1.2046 & 0.3899 & 0.4256 & 94.2034 & 0.0042 & 0.2807 & -41.7350 & -0.1213 & 0.0058 & -1.7718 \\
\hline$\infty \times$ & & $\$ .0602$ & 0.0000 & 0.0000 & 281.8084 & 0.0000 & 0.0000 & -48.8200 & 0.0000 & 0.0000 & 0.0000 \\
\hline 56203 & & 1.1970 & 0.0000 & 0.0000 & 291.4982 & 0.0000 & 0.0000 & 0.0000 & 0.0000 & 0.0000 & 0.0000 \\
\hline P2OS & 0.0400 & 2.2910 & 0.0816 & 0.0975 & 141.9370 & 0.0006 & 0.0442 & -53.6200 & -0.0237 & 0.0000 & -0.3462 \\
\hline nteos & & 1.1660 & 0.0000 & 0.0000 & 336.4782 & 0.0000 & 0.0000 & -28.3200 & 0.0000 & 0.0000 & 0.0000 \\
\hline$L 200$ & & 1.1728 & 0.0000 & 0.0000 & 325.8100 & 0.0000 & 0.0000 & .31 .1400 & 0.0000 & 0.0000 & 0.0000 \\
\hline reos & & 1.2699 & 0.0000 & 0.0000 & 225.8082 & 0.0000 & 0.0000 & -12.9500 & 0.0000 & 0.0000 & 0.0000 \\
\hline $\mathrm{B} O$ & 0.0080 & 1.1185 & 0.0089 & 0.0095 & 153.3394 & $0.000 !$ & 0.0040 & -30.5700 & -0.0012 & 0.0001 & -0.0178 \\
\hline$P \infty$ & & 1.0772 & 0.0000 & 0.0000 & 223.1900 & 0.0000 & 0.0000 & -8.8100 & 0.0000 & 0.0000 & 0.0000 \\
\hline$\infty \infty$ & & 1.2284 & 0.0000 & 0.0000 & 172.1200 & 0.0000 & 0.0000 & 11.3450 & 0.0000 & 0.0000 & 0.0000 \\
\hline Moss & & 1.5003 & 0.0000 & 0.0000 & 143.9382 & 0.0000 & 0.0000 & -21.7500 & 0.0000 & 0.0000 & 0.0000 \\
\hline zro & 0.093 & 1.2447 & 0.1158 & 0.1232 & 81.3800 & 0.0014 & 0.0974 & -2.4000 & -0.0023 & 0.0019 & .0 .0341 \\
\hline$\infty$ & & 1.2518 & 0.0000 & 0.0000 & 79.5454 & 0.0000 & 0.0000 & 1.7900 & 0.0000 & 0.0000 & 0.0000 \\
\hline $\mathrm{Fe2}+/ \mathrm{Fe3}+$ & 0 & & & & & & & & & & \\
\hline $\mathrm{Cl}$ & 0.4591 & & 0.4591 & 0.4591 & & & & & & & \\
\hline$F$ & 0.0259 & & 0.0259 & 0.0259 & & & & & & & \\
\hline SO4 & 0.1601 & & 0.1601 & 0.1801 & & & & & & & \\
\hline \multirow[t]{2}{*}{ OODE SUMS } & 48.3470 & & 83.8684 & 100.0000 & & 1.4603 & 100.0000 & \multirow{3}{*}{$\begin{array}{c}S U M+p H S i \\
S U M+p H B \\
p H=\end{array}$} & .5 .0997 & 0.4250 & .74 .4734 \\
\hline & & & \multirow[b]{2}{*}{ MOLAR SUMB } & & NoRinneso & & & & .5 .0997 & & \\
\hline \multicolumn{2}{|c|}{ MULAR BALANCE (M) } & & & MOLE\% & MOLE & & & & -5.0997 & & \\
\hline $\mathrm{KeSO4}$ & 0.2904 & & SALTS & 1.2982 & 1.1097 & & & & & & \\
\hline $\mathrm{KCl}$ & 0.9648 & & BORATES & 21,4289 & 18.3171 & & & & & & \\
\hline MBF4 & 0.0429 & & CHPOMATES & 0.2928 & 0.2502 & & & & & & \\
\hline $\mathrm{NaAOS}$ & 21.4289 & & OXDES & 93.8684 & 80.3230 & & & & & & \\
\hline \multirow[t]{2}{*}{$\mathrm{Ne} 2 \mathrm{CrO} 4$} & 0.2928 & & & & & & & & & & \\
\hline & & & TOTAL & 116.8883 & 100.0000 & & & & & & \\
\hline K excess(M) & -0.01 & & & & & & & & & & \\
\hline Nh excess (M) & 0.00 & & & & & & & & & & \\
\hline s04 oxcess (i) & 0.00 & & & & & & & & & & \\
\hline CI oxeose & 0.00 & & & & & & & & & & \\
\hline F exces & 0.00 & & & & & & & & & & \\
\hline 8 oxceses & 0.00 & & & & & & & & & & \\
\hline ALKSIO2 = & & 0.391 & & TEST $\mathbf{x}$ & & & & & & & \\
\hline ALK+ B2O3rsioz & & 0.596 & & TIME= & & & & & & & \\
\hline 82038102 = & & 0.205 & & & & & & & & & \\
\hline viscosm eTe & $P(\cdot C)$ & 42.626 & & SA $/ V=$ & & & & & & & \\
\hline LOUDUS RATIO & & 0.079 & & & & & & & & & \\
\hline LOUMUS ("G) = & 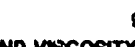 & 49.4943159 & ( & 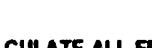 & & & & & & & \\
\hline
\end{tabular}

(SPREAOSHEET REVISION 5.0, OCTOBER 23, 1990) 
GLASS ID $=2 / 3+0$ CENTER - B (ADS *200038843)

\begin{tabular}{|c|c|c|c|c|c|c|c|c|c|c|c|}
\hline & $\begin{array}{l}\text { ELEMENT } \\
\text { WT\% }\end{array}$ & $\begin{array}{l}\text { GRAV } \\
\text { FACTOA }\end{array}$ & $\begin{array}{l}\text { OXIDE } \\
\text { WI\% }\end{array}$ & $\begin{array}{l}\text { NOPMOXIDE } \\
\text { WT } \%\end{array}$ & M.W. & $\begin{array}{c}\text { MOLE } \\
\text { FRACTION }\end{array}$ & $\begin{array}{l}\text { NOPMODDE } \\
\text { MOLE } \%\end{array}$ & $\begin{array}{l}\text { COMPONENT } \\
\text { FPEIENGRGY }\end{array}$ & $\begin{array}{l}\text { FREEEEFGY } \\
\text { HYORATON }\end{array}$ & NBO & KCAL/KG \\
\hline $\mathrm{A} 12 \mathrm{O} 3$ & 1.4780 & 1.8895 & 2.7927 & 3.1264 & 101.9600 & 0.0274 & 1.9473 & 3.0400 & 0.0592 & .0 .0389 & 0.8327 \\
\hline$\infty$ & 0.8550 & 1.3992 & 1.1963 & 1.3393 & 56.0784 & 0.0213 & 1.5166 & -16.1160 & -0.2444 & 0.0303 & -3.4380 \\
\hline F•203 & 6.0560 & 1.4297 & 8.6583 & 9.6830 & 159.6922 & 0.0542 & 3.8546 & 15.5000 & 0.5975 & -0.0771 & 8.4039 \\
\hline$F$ & N/A & 1.2865 & 0.0000 & 0.0000 & 71.8464 & 0.0000 & 0.0000 & -14.6090 & 0.0000 & 0.0000 & 0.0000 \\
\hline$N \infty$ & 0.4590 & 1.6583 & 0.7612 & 0.8521 & 40.3114 & 0.0188 & 1.3424 & -13.8880 & -0.1864 & 0.0268 & -2.6223 \\
\hline Mho & 1.3270 & 1.2912 & 1.7134 & 1.9182 & 70.8374 & 0.0242 & 1.7172 & -14.8710 & -0.2554 & 0.0343 & -3.5919 \\
\hline $\mathrm{Na2O}$ (CP) & 7.9480 & 1.3480 & 10.7139 & 11.8944 & 81.9790 & 0.1729 & 12.2896 & -28.8150 & -3.5412 & 0.2458 & .49 .8106 \\
\hline Li2O & 1.9150 & 2.1525 & 4.1220 & 4.6147 & 29.8774 & 0.1380 & 9.8085 & -22.7400 & -2.2305 & 0.1962 & .31 .3733 \\
\hline NOOO & 0.5690 & 1.2726 & 0.7241 & 0.8107 & 74.7094 & 0.0097 & 0.6891 & -14.3470 & -0.0988 & 0.0138 & -1.3906 \\
\hline SiO2 (ICP) & 21.5640 & 2.1393 & 46.1318 & 51.6453 & 60.0848 & 0.7678 & 54.5847 & $\mathbf{5 . 5 9 0 0}$ & 1.4771 & 0.0000 & 207763 \\
\hline $\mathrm{Cr} 2 \mathrm{O} 3$ & 0.0670 & 1.4616 & 0.0979 & 0.1098 & 151.9874 & 0.0008 & 0.0458 & 37.3600 & 0.0171 & -0.0009 & 02407 \\
\hline B203 & 3.4260 & 3.2201 & 11.0321 & 12.3505 & 69.6204 & 0.1585 & 11.2656 & -9.9300 & -1.1187 & 0.0000 & -157351 \\
\hline Uer & & 1.1344 & 0.0000 & 0.0000 & 270.0388 & 0.0000 & 0.0000 & -6.8000 & 0.0000 & 0.0000 & 0.0000 \\
\hline mos & & 1.1379 & 0.0000 & 0.0000 & 264.0400 & 0.0000 & 0.0000 & -2.5330 & 0.0000 & 0.0000 & 0.0000 \\
\hline sio & 0.0350 & 1.1826 & 0.0414 & 0.0463 & 103.6194 & 0.0004 & 0.0284 & -24.4000 & -0.0069 & 0.0006 & $\cdot 0.0975$ \\
\hline 202 & 0.4670 & 1.3508 & 0.6308 & 0.7062 & 123.2188 & 0.0051 & 0.3640 & 45.1000 & 0.1642 & 0.0146 & 2.3088 \\
\hline TiO2 & $0.074 J$ & 1.6680 & 0.1234 & 0.1382 & 79.8988 & 0.0015 & 0.1098 & 15.9900 & 0.0176 & 0.0044 & 0.2470 \\
\hline 120 & 0.3320 & 1.2046 & 0.3999 & 0.4477 & 94.2034 & 0.0042 & 0.3018 & -41.7350 & -0.1260 & 0.0060 & -1.7718 \\
\hline 020 & & 1.0602 & 0.0000 & 0.0000 & 281.8094 & 0.0000 & 0.0000 & -46.8200 & 0.0000 & 0.0000 & 0.0000 \\
\hline $\mathbf{S 0 2 0 3}$ & & 1.1970 & 0.0000 & 0.0000 & 291.4982 & 0.0000 & 0.0000 & 0.0000 & 0.0000 & 0.0000 & 0.0000 \\
\hline P2OS & 0.0270 & 2.2910 & 0.0619 & 0.0692 & 141.9370 & 0.0004 & 0.0310 & -53.6200 & -0.0166 & 0.0000 & -0.2337 \\
\hline$N+203$ & & 1.1660 & 0.0000 & 0.0000 & 336.4782 & 0.0000 & 0.0000 & -28.3200 & 0.0000 & 0.0000 & 0.0000 \\
\hline$L 203$ & & 1.1728 & 0.0000 & 0.0000 & 925.8100 & 0.0000 & 0.0000 & -31.1400 & 0.0000 & 0.0000 & 0.0000 \\
\hline $\mathrm{roO}$ & & 1.2699 & 0.0000 & 0.0000 & 225.8082 & 0.0000 & 0.0000 & -12.9500 & 0.0000 & 0.0000 & 0.0000 \\
\hline$E$ & 0.0090 & 1.1165 & 0.0100 & 0.0112 & 153.3394 & 0.0001 & 0.0047 & -30.5700 & -0.0014 & 0.0001 & -0.0200 \\
\hline$P \infty O$ & & 1.0772 & 0.0000 & 0.0000 & 223.1900 & 0.0000 & 0.0000 & -8.8100 & 0.0000 & 0.0000 & 0.0000 \\
\hline $\cos$ & & 1.2284 & 0.0000 & 0.0000 & 172.1200 & 0.0000 & 0.0000 & 11.3450 & 0.0000 & 0.0000 & 0.0000 \\
\hline $\operatorname{mos}$ & & 1.5003 & 0.0000 & 0.0000 & 143.9382 & 0.0000 & 0.0000 & -21.7500 & 0.0000 & 0.0000 & 0.0000 \\
\hline 20 & 0.091 & 1.2447 & 0.1133 & 0.1268 & 81.3800 & 0.0014 & 0.0990 & -2.4000 & -0.0024 & 0.0020 & -0.0334 \\
\hline$\infty$ & & 1.2518 & 0.0000 & 0.0000 & 79.5454 & 0.0000 & 0.0000 & 1.7900 & 0.0000 & 0.0000 & 0.0000 \\
\hline $\mathrm{F} \bullet 2+1 \mathrm{~F} \bullet 3+$ & 0 & & & & & & & & & & \\
\hline $\mathrm{Cl}$ & 0.4091 & & 0.4091 & 0.4091 & & & & & & & \\
\hline $\mathbf{F}$ & 0.0187 & & 0.0187 & 0.0187 & & & & & & & \\
\hline sod & 0.1467 & & 0.1467 & 0.9467 & & & & & & & \\
\hline \multirow[t]{2}{*}{ ONDE SUMS } & 46.6990 & & 89.3245 & 100.0000 & & 1.4066 & 100.0000 & & .5 .4962 & 0.4579 & -77.3087 \\
\hline & & & \multirow[b]{2}{*}{ MOLAR SUms } & & MORMLALZED & & & \multirow{2}{*}{$\begin{array}{c}S U M+p H S i \\
S U M+p H B \\
p H=\end{array}$} & -5.4962 & & \\
\hline \multicolumn{2}{|c|}{ MLLAR BALANCE (M) } & & & Mate\% & MOLE* & & & & -5.4962 & & \\
\hline K2SO4 & 0.2661 & & SALTS & 1.1568 & 1.0372 & & & & & & \\
\hline $\mathrm{KCl}$ & 0.8597 & & BOFUTES & 20.8507 & 18.6934 & & & & & & \\
\hline KBF4 & 0.0310 & & CHPOMATES & 0.2087 & 0.1871 & & & & & & \\
\hline NeBOe & 20.8507 & & OXIDES & 89.3245 & 80.0824 & & & & & & \\
\hline \multirow[t]{2}{*}{$\mathrm{NazCrO} 4$} & 0.2087 & & & & & & & & & & \\
\hline & & & TOTAL. & 111.5408 & 100.0000 & & & & & & \\
\hline Kexeeses(i) & $\begin{array}{r}-0.01 \\
0.03\end{array}$ & & & & & & & & & & \\
\hline so4 oxcens (M) & 0.00 & & & & & & & & & & \\
\hline C1 excess & 0.00 & & & & & & & & & & \\
\hline F excoses & 0.00 & & & & & & & & & & \\
\hline B excoss: & 0.00 & & & & & & & & & & \\
\hline ALK/SIO2 = & & 0.410 & & TEST= & & & & & & & \\
\hline ALK+ B203/S10 & & 0.617 & & TIME $=$ & & & & & & & \\
\hline $8203 / 8102=$ & & 0.206 & & & & & & & & & \\
\hline VSCOSITY QTE & $P\left({ }^{\circ} \mathrm{C}\right)$ & 38.481 & & SA/V= & & & & & & & \\
\hline LOUIDUS RATIO & & 0.066 & & & & & & & & & \\
\hline $\begin{array}{l}\text { LOUIDUS }\left({ }^{\circ} \mathrm{C}\right)= \\
\text { NoTE LaUnDUS }\end{array}$ & sumonon & 0.6307776 & 1) & 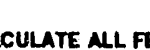 & 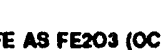 & $x^{-1}$ & & & & & \\
\hline
\end{tabular}

(SPREADSHEET REVISION 5.0, DCTOBEA 23, 1990) 
GLASS ID= 2/3 + 6 EDGE - A (ADS 200038844)

\begin{tabular}{|c|c|c|c|c|c|c|c|c|c|c|c|}
\hline & $\begin{array}{c}\text { EEMENT } \\
\text { WT\% }\end{array}$ & $\begin{array}{l}\text { GRAV } \\
\text { FACTOR }\end{array}$ & $\begin{array}{l}\text { OXIDE } \\
\text { WT\% }\end{array}$ & $\begin{array}{l}\text { NOFMOXIDE } \\
\text { WT \% }\end{array}$ & M.W. & $\begin{array}{c}\text { MOLE } \\
\text { FPACTION }\end{array}$ & $\begin{array}{l}\text { NOPM OXIDE } \\
\text { MOEE } \%\end{array}$ & $\begin{array}{l}\text { COMPONENT } \\
\text { FPEE ENEAGYY }\end{array}$ & $\begin{array}{l}\text { FREEENERGY } \\
\text { HYDRATION }\end{array}$ & NBO & KCAL/KG \\
\hline $\mathrm{A} 1203$ & 2.8080 & 1.8895 & 5.3057 & 5.3766 & 101.9600 & 0.0520 & 3.4089 & 3.0400 & 0.1036 & -0.0682 & 1.5819 \\
\hline$\infty$ & 0.9910 & 1.3992 & 1.3866 & 1.4051 & 56.0794 & 0.0247 & 1.6198 & -16.1160 & -0.2610 & 0.0324 & -3.9848 \\
\hline Fo203 & 7.3330 & 1.4297 & 10.4840 & 10.6241 & 159.6922 & 0.0657 & 4.3008 & 155000 & 0.6666 & -0.0860 & 10.1759 \\
\hline$F \infty$ & N/A & 1.2865 & 0.0000 & 0.0000 & 71.8464 & 0.0000 & 0.0000 & -14.6090 & 0.0000 & 0.0000 & 0.0000 \\
\hline MoO & 0.5010 & 1.6583 & 0.8308 & 0.8419 & 40.3114 & 0.0206 & 1.3501 & -13.8880 & -0.1875 & 0.0270 & -2.8823 \\
\hline MNO & 1.6050 & 1.2912 & 2.0724 & 2.1001 & 70.9374 & 0.0292 & 1.9138 & -14.8710 & -0.2846 & 0.0383 & -4.3444 \\
\hline$N=20$ & 8.5030 & 1.3480 & 11.4620 & 11.6152 & 61.9790 & 0.1849 & 12.1150 & -28.8150 & -3.4809 & 0.2423 & .53 .2888 \\
\hline Li2O & 2.0020 & 2.1525 & 4.3093 & 4.3669 & 29.8774 & 0.1442 & 9.4486 & -22.7400 & -2.1486 & 0.1890 & -32.7986 \\
\hline NO & 0.6920 & 1.2726 & 0.8806 & 0.8924 & 74.7094 & 0.0118 & 0.7722 & -14.3470 & -0.1108 & 0.0154 & -1.6812 \\
\hline SiO2 & 22.8500 & 2.1393 & 48.8830 & 48.5361 & 60.0848 & 0.8136 & 53.2964 & 5.5900 & 1.4177 & 0.0000 & 21.6411 \\
\hline $\mathrm{Cr}_{2} \mathrm{OO}$ & 0.0760 & 1.4616 & 0.1111 & 0.1126 & 151.8974 & 0.0007 & 0.0479 & 37.3600 & 0.0179 & -0.0010 & 0.2730 \\
\hline 8203 & 3.5760 & 3.2201 & 11.5151 & 11.6689 & 69.6204 & 0.1654 & 10.8351 & -9.9300 & .1 .0759 & 0.0000 & -16.4240 \\
\hline Ler & & 1.1344 & 0.0000 & 0.0000 & 270.0388 & 0.0000 & 0.0000 & -6.8000 & 0.0000 & 0.0000 & 0.0000 \\
\hline ThO2 & & 1.1379 & 0.0000 & 0.0000 & 264.0400 & 0.0000 & 0.0000 & -2.5330 & 0.0000 & 0.0000 & 0.0000 \\
\hline sro & 0.0390 & 1.1826 & 0.0461 & 0.0467 & 103.6194 & 0.0004 & 0.0292 & -24.4000 & -0.0071 & 0.0006 & -0.1086 \\
\hline 202 & 0.1710 & 1.3508 & 0.6362 & 0.6447 & 123.2188 & 0.0052 & 0.3383 & 45.1000 & 0.1526 & 0.0135 & 2.3287 \\
\hline $\mathrm{THO} 2$ & 0.0830 & 1.6680 & 0.1384 & 0.1403 & 79.8988 & 0.0017 & 0.1135 & 15.8900 & 0.0182 & 0.0045 & 0.2771 \\
\hline 120 & 0.2920 & 1.2046 & 0.3517 & 0.3564 & 94.2034 & 0.0037 & 0.2446 & -41.7350 & -0.1021 & 0.0049 & -1.5583 \\
\hline 0020 & & 1.0602 & 0.0000 & 0.0000 & 281.8094 & 0.0000 & 0.0000 & .46 .8200 & 0.0000 & 0.0000 & 0.0000 \\
\hline $\mathrm{Sb} 203$ & & 1.1970 & 0.0000 & 0.0000 & 281.4982 & 0.0000 & 0.0000 & 0.0000 & 0.0000 & 0.0000 & 0.0000 \\
\hline PZOS & 0.0590 & 2.2910 & 0.1352 & 0.1370 & 141.9370 & 0.0010 & 0.0624 & -53.6200 & .0 .0335 & 0.0000 & .0 .5106 \\
\hline $\mathrm{N}+2 \mathrm{OS}$ & & 1.1660 & 0.0000 & 0.0000 & 336.4782 & 0.0000 & 0.0000 & .28 .3200 & 0.0000 & 0.0000 & 0.0000 \\
\hline $\mathrm{L} 203$ & & 1.1726 & 0.0000 & 0.0000 & 325.8100 & 0.0000 & 0.0000 & -31.1400 & 0.0000 & 0.0000 & 0.0000 \\
\hline r203 & & 1.2699 & 0.0000 & 0.0000 & 225.8082 & 0.0000 & 0.0000 & -12.9500 & 0.0000 & 0.0000 & 0.0000 \\
\hline $8 \infty 0$ & 0.0080 & 1.1165 & 0.0100 & 0.0102 & 153.3394 & 0.0001 & 0.0043 & -30.5700 & .0 .0013 & 0.0001 & .0 .0200 \\
\hline$P \infty O$ & & 1.0772 & 0.0000 & 0.0000 & 223.1900 & 0.0000 & 0.0000 & -8.8100 & 0.0000 & 0.0000 & 0.0000 \\
\hline $\cos \infty$ & & 1.2284 & 0.0000 & 0.0000 & 172.1200 & 0.0000 & 0.0000 & 11.3450 & 0.0000 & 0.0000 & 0.0000 \\
\hline MoOs & & 1.5003 & 0.0000 & 0.0000 & 143.8382 & 0.0000 & 0.0000 & -21.7500 & 0.0000 & 0.0000 & 0.0000 \\
\hline $2 n$ & 0.099 & 1.2447 & 0.1232 & 0.1249 & 81.3800 & 0.0015 & 0.0992 & -2.4000 & .0 .0024 & 0.0020 & .0 .0363 \\
\hline a & & 1.2518 & 0.0000 & 0.0000 & 79.5454 & 0.0000 & 0.0000 & 1.7900 & 0.0000 & 0.0000 & 0.0000 \\
\hline $\mathrm{F}_{\theta 2}+\mathrm{F} \mathrm{F}+$ & 0 & & & & & & & & & & \\
\hline $\mathrm{Cl}$ & 0.3914 & & 0.3914 & 0.3914 & & & & & & & \\
\hline $\mathbf{F}$ & 0.0229 & & 0.0229 & 0.0229 & & & & & & & \\
\hline som & 0.1597 & & 0.1597 & 0.1597 & & & & & & & \\
\hline \multirow[t]{2}{*}{ OCOE SUMS } & 51.9890 & & 98.6816 & 100.0000 & & 1.5265 & 100.0000 & & .5 .3292 & 0.4149 & .81 .3502 \\
\hline & & & & & NORMALZED & & & $\mathrm{SUM}+\mathrm{pH} \mathrm{Si}$ & -5.3292 & & \\
\hline \multirow{2}{*}{\multicolumn{2}{|c|}{ MOLAR BALANCE (M) }} & & MOLAR SUMS & MOLEX & MOLEX & & & $S U M+P H B$ & -5.3292 & & \\
\hline & & & & & 0041 & & & $\mathrm{pH}=$ & & & \\
\hline $\mathrm{KCl}$ & & & & & & & & & & & \\
\hline & ofeng & & & 21.1000 & 17.0030 & & & & & & \\
\hline KBF4 & 0.0379 & & CHFOMATES & 0.2367 & 0.1943 & & & & & & \\
\hline $\mathrm{N}=0 \mathrm{R}$ & 21.7636 & & OXDES & 98.6816 & 80.9980 & & & & & & \\
\hline \multirow[t]{2}{*}{$\mathrm{N} 22 \mathrm{CrO} 4$} & 0.2367 & & & & & & & & & & \\
\hline & & & TOTAL & 121.8322 & 100.0000 & & & & & & \\
\hline K oxcoses(M) & -0.01 & & & & & & & & & & \\
\hline Na excoss (M) & 0.04 & & & & & & & & & & \\
\hline sos exceses (M & 0.00 & & & & & & & & & & \\
\hline CI exceses & 0.00 & & & & & & & & & & \\
\hline F oxcoses & 0.00 & & & & & & & & & & \\
\hline B excess & 0.00 & & & & & & & & & & \\
\hline ALK/SIO2 = & & 0.409 & & TEST $=$ & & & & & & & \\
\hline $\mathrm{ALK}+\mathrm{B2O} 3 / \mathrm{SHO} 2$ & & 0.612 & & TIME= & & & & & & & \\
\hline B203/S102 = & & 0.203 & & & & & & & & & \\
\hline VISCOSTY QTER & $\mid P\left({ }^{\circ} \mathrm{C}\right)$ & 45.073 & & SA/V $=$ & & & & & & & \\
\hline $\begin{array}{l}\text { LOUDUS RATIO } \\
\text { HOUDUS ("C) = }\end{array}$ & & $\begin{array}{c}0.082 \\
59.1188304\end{array}$ & & & & & & & & & \\
\hline NOTE LQUDDUS & (5) & - & 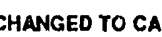 & 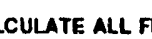 & & & & & & & \\
\hline
\end{tabular}

(SPREADSHEET REVISION 5,0, OCTOBER 23, 1990) 
GLASS ID $=2 / 3+6$ EDGE $-B($ (ADS $\$ 200038845)$

\begin{tabular}{|c|c|c|c|c|c|c|c|c|c|c|c|}
\hline & $\begin{array}{l}\text { ELEMENT } \\
\text { WT* }\end{array}$ & $\begin{array}{l}\text { GRAV } \\
\text { FACTOR }\end{array}$ & $\begin{array}{l}\text { OXIDE } \\
\text { WT\% }\end{array}$ & $\begin{array}{l}\text { NOPM OXIDE } \\
\text { WT\% }\end{array}$ & M.W. & $\begin{array}{c}\text { MOLE } \\
\text { FRACTION }\end{array}$ & $\begin{array}{l}\text { NOPMCXIDE } \\
\text { MOLE \% }\end{array}$ & $\begin{array}{l}\text { CONPONENT } \\
\text { FREIENERGY }\end{array}$ & $\begin{array}{l}\text { FREEQNERGY } \\
\text { HYDFATION }\end{array}$ & NBO & KCALKG \\
\hline A|2O3 & 1.7430 & 1.8895 & 3.2934 & 3.4534 & 101.9600 & 0.0323 & 2.1729 & $\quad 3.0400$ & 0.0661 & .0 .0435 & 0.9819 \\
\hline$\infty$ & 0.8810 & 1.3992 & 1.2327 & 1.2926 & 56.0794 & 0.0220 & 1.4787 & -16.1160 & -0.2383 & 0.0296 & -3.5425 \\
\hline $\mathrm{F} 0203$ & 7.2690 & 1.4297 & 10.3925 & 10.8973 & 159.6922 & 0.0651 & 4.3779 & 15.5000 & 0.6786 & $\cdot 0.0876$ & 10.0871 \\
\hline$F \infty$ & $N / A$ & 1.2865 & 0.0000 & 0.0000 & 71.8464 & 0.0000 & 0.0000 & -14.6090 & 0.0000 & 0.0000 & 0.0000 \\
\hline$N \infty$ & 0.4860 & 1.6583 & 0.8059 & 0.8451 & 40.3114 & 0.0200 & 1.3449 & -13.8880 & -0.1868 & 0.0269 & -2.7766 \\
\hline Mo & 1.5690 & 1.2912 & 2.0259 & 2.1243 & 70.8374 & 0.0286 & 1.9212 & -14.8710 & -0.2857 & 0.0384 & -4.2470 \\
\hline$N=20$ & 8.2920 & 1.3480 & 11.1776 & 11.7206 & 61.9790 & 0.1803 & 12.1320 & -28.8150 & -3.4958 & 0.2426 & -519665 \\
\hline Li2O & 2.0020 & 2.1525 & 4.3093 & 4.5186 & 29.8774 & 0.1442 & 9.7027 & $\cdot 22.7400$ & -2.2064 & 0.1941 & .327986 \\
\hline NO & 0.6740 & 1.2726 & 0.8577 & 0.8994 & 74.7094 & 0.0115 & 0.7723 & -14.3470 & -0.1108 & 0.0154 & -16472 \\
\hline $\mathrm{SiO} 2$ & 22.5620 & 2.1393 & 48.2669 & 50.6115 & 60.0848 & 0.8033 & 54.0397 & 5.5900 & 1.4476 & 0.0000 & 21.5192 \\
\hline $\mathrm{Cr}_{2} \mathrm{O}_{3}$ & 0.0650 & 1.4616 & 0.0950 & 0.0996 & 151.9974 & 0.0006 & 0.0420 & 37.3600 & 0.0157 & -0.0008 & 0.2335 \\
\hline $\mathrm{B203}$ & 3.5650 & 3.2201 & 11.4797 & 12.0373 & 69.6204 & 0.1649 & 11.0923 & -9.9300 & -1.1015 & 0.0000 & -16.3735 \\
\hline we & & 1.1344 & 0.0000 & 0.0000 & 270.0388 & 0.0000 & 0.0000 & .6 .8000 & 0.0000 & 0.0000 & 0.0000 \\
\hline ThOs & & 1.1379 & 0.0000 & 0.0000 & 264.0400 & 0.0000 & 0.0000 & -2.5330 & 0.0000 & 0.0000 & 0.0000 \\
\hline sro & 0.0350 & 1.1826 & 0.0414 & 0.0434 & 103.6194 & 0.0004 & $0.026 \theta$ & -24.4000 & -0.0066 & 0.0005 & -0.0975 \\
\hline $2 \mathrm{rO2}$ & 0.4950 & 1.3508 & 0.6686 & 0.7011 & 123.2188 & 0.0054 & 0.3650 & 45.1000 & 0.1846 & 0.0146 & 2.4473 \\
\hline TiO2 & 0.0770 & 1.6680 & 0.1284 & 0.1347 & 79.8988 & 0.0016 & 0.1081 & 15.9900 & 0.0173 & 0.0043 & 0.2570 \\
\hline$k \geq 0$ & 0.3400 & 1.2046 & 0.4096 & 0.4295 & 94.2034 & 0.0043 & 0.2925 & -41.7350 & -0.1221 & 0.0058 & -1.8145 \\
\hline $\cos 0$ & & 1.0602 & 0.0000 & 0.0000 & 281.8094 & 0.0000 & 0.0000 & -46.8200 & 0.0000 & 0.0000 & 0.0000 \\
\hline $\mathrm{Sb2O3}_{3}$ & & 1.1970 & 0.0000 & 0.0000 & 291.4982 & 0.0000 & 0.0000 & 0.0000 & 0.0000 & 0.0000 & 0.0000 \\
\hline P2OS & 0.0220 & 2.2910 & 0.0504 & 0.0529 & 141.8370 & 0.0004 & 0.0239 & -53.6200 & -0.0128 & 0.0000 & -0.1904 \\
\hline $\mathrm{N}+2 \mathrm{O} 3$ & & 1.1660 & 0.0000 & 0.0000 & 336.4782 & 0.0000 & 0.0000 & -28.3200 & 0.0000 & 0.0000 & 0.0000 \\
\hline $\mathrm{LazO}$ & & 1.1728 & 0.0000 & 0.0000 & 325.8100 & 0.0000 & 0.0000 & -31.1400 & 0.0000 & 0.0000 & 0.0000 \\
\hline $\mathrm{raO}$ & & 1.2699 & 0.0000 & 0.0000 & 225.8082 & 0.0000 & 0.0000 & -12.9500 & 0.0000 & 0.0000 & 0.0000 \\
\hline$B=0$ & 0.0060 & 1.1165 & 0.0067 & 0.0070 & 153.3394 & 0.0000 & 0.0029 & -30.5700 & .0 .0009 & 0.0001 & -0.0134 \\
\hline$P \infty$ & & 1.0772 & 0.0000 & 0.0000 & 223.1900 & 0.0000 & 0.0000 & -8.8100 & 0.0000 & 0.0000 & 0.0000 \\
\hline $\cos 2$ & & 1.2284 & 0.0000 & 0.0000 & 172.1200 & 0.0000 & 0.0000 & 11.3450 & 0.0000 & 0.0000 & 0.0000 \\
\hline MoOS & & 1.5003 & 0.0000 & 0.0000 & 143.9382 & 0.0000 & 0.0000 & -21.7500 & 0.0000 & 0.0000 & 0.0000 \\
\hline 270 & 0.101 & 1.2447 & 0.1257 & 0.1318 & 81.3800 & 0.0015 & 0.1039 & -2.4000 & -0.0025 & 0.0021 & -0.0371 \\
\hline 0 & & 1.2518 & 0.0000 & 0.0000 & 79.5454 & 0.0000 & 0.0000 & 1.7900 & 0.0000 & 0.0000 & 0.0000 \\
\hline $\mathrm{F} \bullet 2+1 \mathrm{~F}_{\theta 3+}$ & 0 & & & & & & & & & & \\
\hline $\mathrm{Cl}$ & 0.4284 & & 0.4284 & 0.4284 & & & & & & & \\
\hline$F$ & 0.0233 & & 0.0233 & 0.0233 & & & & & & & \\
\hline sod & 0.1698 & & 0.1698 & 0.1698 & & & & & & & \\
\hline \multirow[t]{2}{*}{ OXDE SUMS } & 50.1840 & & 95.3675 & 100.0000 & & 1.4865 & 100.0000 & & .5 .3802 & 0.4426 & .79 .9784 \\
\hline & & & & & NORMALZED & & & $S U M+P H S i$ & -5.3802 & & \\
\hline \multicolumn{2}{|c|}{ MOLAP BALANCE (M) } & & MOLAR SUMS & MOLEX & MOLE\% & & & $\begin{array}{c}\mathrm{SUM}+\mathrm{pHB} \\
\mathrm{pH}=\end{array}$ & -5.3802 & & \\
\hline $\mathrm{K} 2 \mathrm{SO}_{4}$ & 0.3080 & & SALTS & 1.2469 & 1.0521 & & & & & & \\
\hline $\mathrm{KCl}$ & 0.9003 & & BOAATES & 21.6967 & 18.3074 & & & & & & \\
\hline KBF4 & 0.0386 & & CHPOMATES & 0.2024 & 0.1708 & & & & & & \\
\hline $\mathrm{NaPO} 2$ & 21.6967 & & OXDES & 95.3675 & 80.4697 & & & & & & \\
\hline \multirow[t]{2}{*}{$\mathrm{Na2CrO} 4$} & 0.2024 & & & & & & & & & & \\
\hline & & & TOTAL & 118.5135 & 100.0000 & & & & & & \\
\hline$K$ excess(M) & .0 .01 & & & & & & & & & & \\
\hline Ne excese (M) & 0.03 & & & & & & & & & & \\
\hline so4 excess (M & 0.00 & & & & & & & & & & \\
\hline Ci excese & 0.00 & & & & & & & & & & \\
\hline F excoss & 0.00 & & & & & & & & & & \\
\hline B excess & 0.00 & & & & & & & & & & \\
\hline ALK/S1O2 = & & 0.408 & & TEST $=$ & & & & & & . & \\
\hline ALK + B2O3/SiO & & 0.615 & & TIME = & & & & & & & \\
\hline $\mathrm{B} 2 \mathrm{O} 3 / \mathrm{S} 102=$ & & 0.205 & & & & & & & & & \\
\hline VIscosity ere & $I P\left({ }^{\circ} \mathrm{C}\right)$ & 37.226 & & $S A / V=$ & & & & & & & \\
\hline UQUIDUS RATIC & & 0.077 & & & & & & & & & \\
\hline LaUIDUS ("C) = & & 044.3934010 & & & & & & & & & \\
\hline
\end{tabular}

(SPREADSHEet ReVISION 5.0, OCTOBEH 23, 1990) 
GLASS 10 $=2 / 3+6$ CENYER - A (ADS \#200038846)

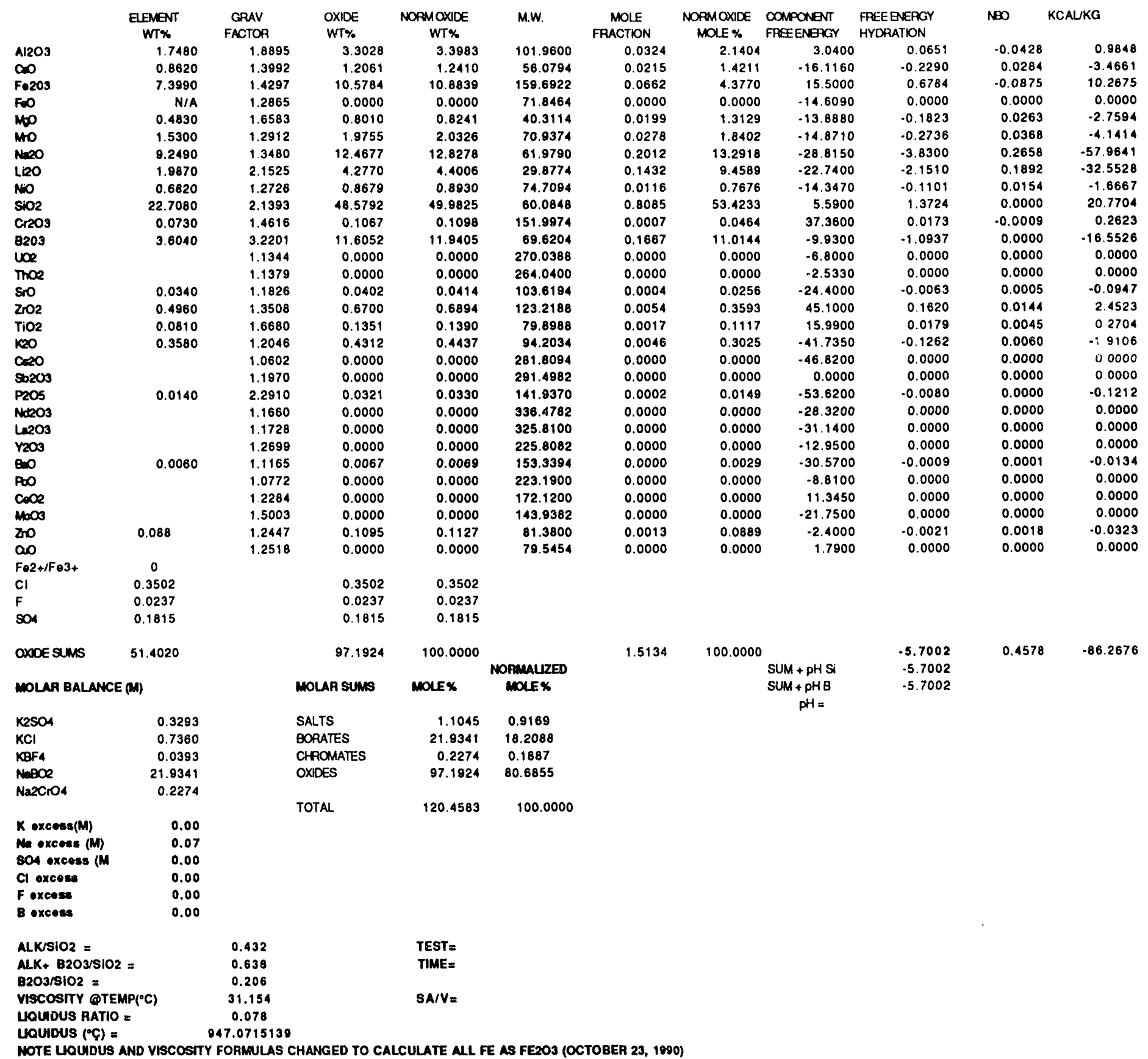

(SPREADSHEET REVISION 5.0, OCTOBER 23, 1990)

(n)


GLASS $10=2 / 3+6$ CENTER - B (ADS \$200038847)

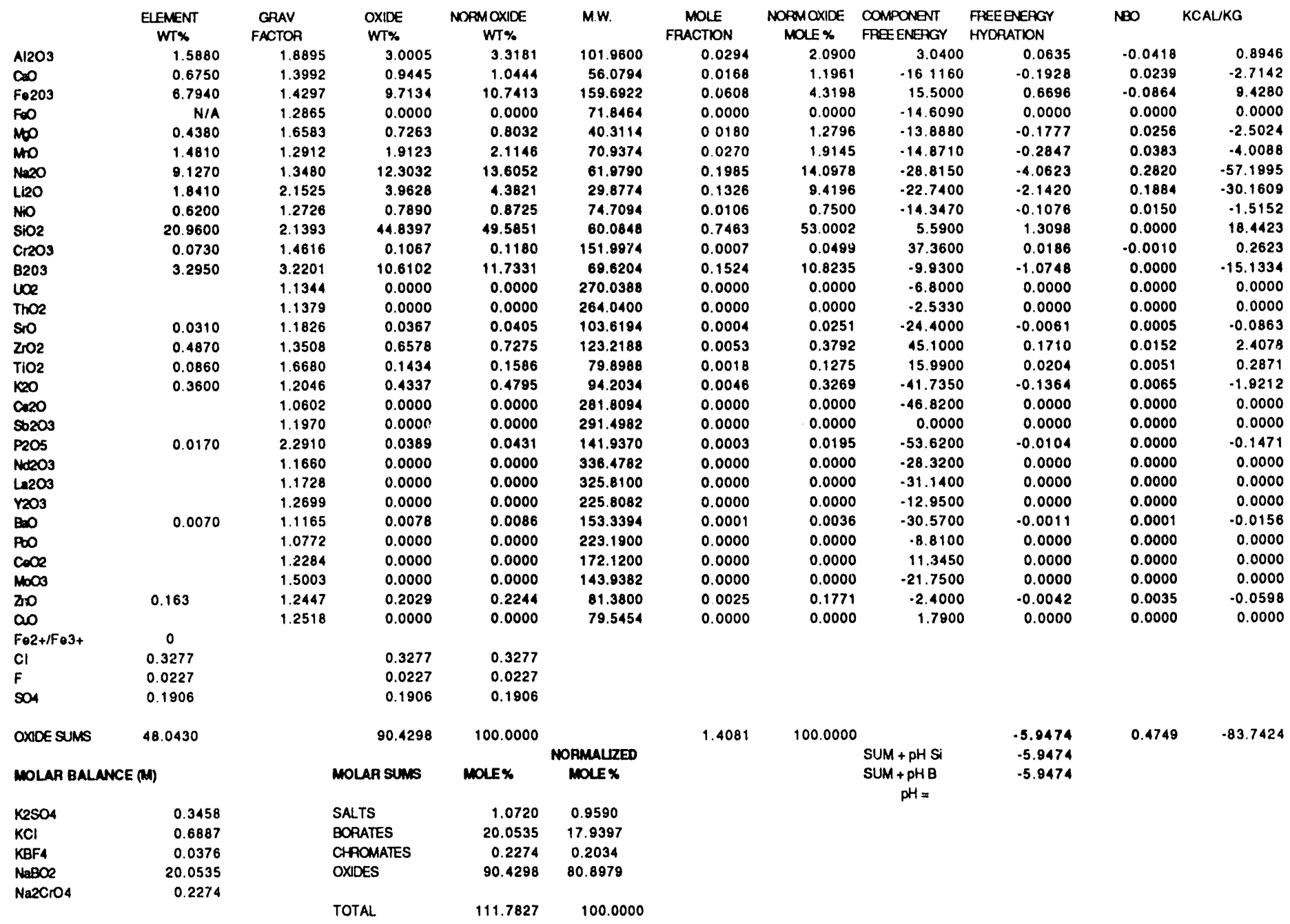

(SPREADSHEET REVISION 5.0, OCTOBER 23, 1990)

MPMOXIDE COMPONENT FREEENERGY NBO KCAL/KG

946

4280

0.0000

.5024

57.1995

30.1609

$-1.5152$

0.2623

.0000

0.0000

.0863

.2871

.0212

0.0000

.0000

.0156

0.0000

.0000

.0598

0000

$\begin{array}{ll}K \text { oxcess }(M) & 0.00 \\ \text { Na excoss }(M) & 0.00 \\ \text { s04 excess (M } & 0.00 \\ \text { CI oxcoss } & 0.00 \\ F \text { excess } & 0.00 \\ \text { excoss } & 0.00\end{array}$

$\begin{array}{lcc}\text { ALKISIO2 }= & 0.450 & \text { TEST }= \\ \text { ALK+ B2O3/SIO2 } & 0.654 & \text { TIME= } \\ \text { B2O3/SIO2 }= & 0.204 & \\ \left.\text { VISCOSITY QTENP( }{ }^{\circ} \mathrm{C}\right) & 27.277 & \text { SA/V }= \\ \text { UQUIDUS RATIO }= & 0.077 & \\ \text { UQUIDUS }\left({ }^{\circ} \mathrm{C}\right)= & 945.3955655 & \end{array}$


GLASS ID $=2 / 3+9$ EDGE - A (ADS *200038848)

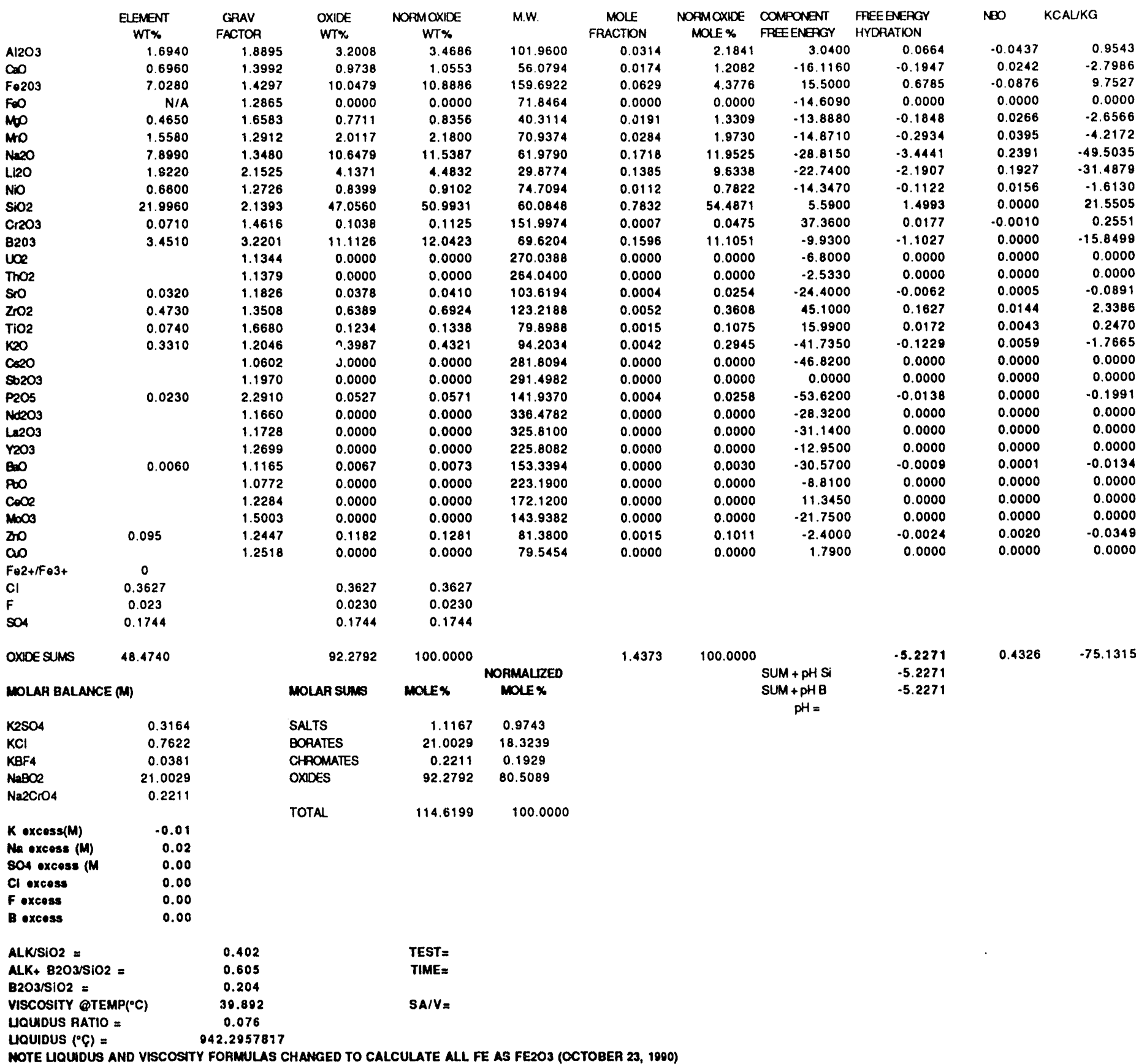
OXIOE COMPONENT FFEE

(SPREADSHEET REVISION 5.0, OCTOBER 23, 1990) 
GLASS ID= 2/.3+ 9 EDGE - B (ADS \#200038049)

\begin{tabular}{|c|c|c|c|c|c|c|c|c|c|c|c|}
\hline & $\begin{array}{l}\text { EENENT } \\
\text { WT\% }\end{array}$ & $\begin{array}{l}\text { GRAV } \\
\text { FACTOR }\end{array}$ & $\begin{array}{l}\text { OXIOE } \\
\text { WT\% }\end{array}$ & $\begin{array}{c}\text { NOPMOXIDE } \\
\text { WT } \%\end{array}$ & M.W. & $\begin{array}{c}\text { MOLE } \\
\text { FRACTION }\end{array}$ & $\begin{array}{l}\text { NOFMOXIDE } \\
\text { MOLE \% }\end{array}$ & $\begin{array}{l}\text { CONPONENT } \\
\text { FREEENERGY }\end{array}$ & $\begin{array}{l}\text { FPEE BNEACY } \\
\text { HYORATION }\end{array}$ & NBO & KCAUKG \\
\hline Al2O3 & 1.6140 & 1.8895 & 3.0497 & 3.3605 & 101.9600 & 0.0299 & 2.1166 & 3.0400 & 0.0643 & .0 .0423 & 0.9093 \\
\hline$\infty$ & 0.6570 & 1.3992 & 0.9193 & 1.0130 & 56.0794 & 0.0164 & 1.1600 & $\cdot 16.1160$ & -0.1869 & 0.0232 & -2.6418 \\
\hline Fo203 & 6.8500 & 1.4297 & 9.7934 & 10.7915 & 159.6922 & 0.0613 & 4.3398 & 15.5000 & 0.6727 & -0.0868 & 9.5057 \\
\hline $\mathrm{FeO}$ & N/A & 1.2865 & 0.0000 & 0.0000 & 71.8464 & 0.0000 & 0.0000 & -14.6090 & 0.0000 & 0.0000 & 0.0000 \\
\hline$\$ 0$ & 0.4360 & 1.6583 & 0.7230 & 0.7967 & 40.3114 & 0.0179 & 1.2692 & -13.8880 & -0.1763 & 0.0254 & -2.4909 \\
\hline Mo & 1.4840 & 1.2912 & 1.9161 & 2.1114 & 70.9374 & 0.0270 & 1.9115 & -14.8710 & -0.2843 & 0.0382 & -4.0169 \\
\hline $\mathrm{N}=20$ & 8.9640 & 1.3480 & 12.0835 & 13.3149 & 61.8780 & 0.1950 & 13.7962 & -28.8150 & -3.9754 & 0.2759 & -56.1779 \\
\hline Li2O & 1.8530 & 2.1525 & 3.9886 & 4.3951 & 29.8774 & 0.1335 & 9.4469 & -22.7400 & -21482 & 0.1889 & .30 .3575 \\
\hline NO & 0.6430 & 1.2726 & 0.8183 & 0.9017 & 74.7094 & 0.0110 & 0.7751 & -14.3470 & -0.1112 & 0.0155 & -1.5714 \\
\hline SiO2 & 21.1280 & 2.1393 & 45.1991 & 19.8055 & 60.0848 & 0.7523 & 53.2328 & $\mathbf{5 . 5 9 0 0}$ & 1.3451 & 0.0000 & 19.0080 \\
\hline $\mathrm{Cr}_{2} \mathrm{O}$ & 0.0620 & 1.4616 & 0.0906 & 0.0999 & 151.9974 & 0.0006 & 0.0422 & 37.3600 & 0.0158 & -0.0008 & 0.2227 \\
\hline 8203 & 3.3530 & 3.2201 & 10.7970 & 11.8973 & 69.6204 & 0.1551 & 10.9744 & -9.9300 & -1.0898 & 0.0000 & -15.3998 \\
\hline we & & 1.1344 & 0.0000 & 0.0000 & 270.0388 & 0.0000 & 0.0000 & .6 .8000 & 0.0000 & 0.0000 & 0.0000 \\
\hline ThO2 & & 1.1379 & 0.0000 & 0.0000 & 264.0400 & 0.0000 & 0.0000 & -2.5330 & 0.0000 & 0.0000 & 0.0000 \\
\hline sro & 0.0290 & 1.1826 & 0.0343 & 0.0378 & 103.6194 & 0.0003 & 0.0234 & -24.4000 & -0.0057 & 0.0005 & -0.0808 \\
\hline $\mathrm{ZOO2}$ & 0.4810 & 1.3508 & 0.6497 & 0.7160 & 123.2188 & 0.0053 & 0.3731 & 45.1000 & 0.1683 & 0.0149 & 2.3781 \\
\hline THO2 & 0.0730 & 1.6680 & 0.1218 & 0.1342 & 79.8988 & 0.0015 & 0.1078 & $\$ 5.9900$ & 0.0172 & 0.0043 & 0.2437 \\
\hline 100 & 0.3350 & 1.2046 & 0.4035 & 0.4447 & 94.2034 & 0.0043 & 0.3031 & -41.7350 & -0.1265 & 0.0081 & -1.7878 \\
\hline 020 & & 1.0602 & 0.0000 & 0.0000 & 281.8094 & 0.0000 & 0.0000 & -46.8200 & 0.0000 & 0.0000 & 0.0000 \\
\hline $\mathrm{Sb}_{203}$ & & 1.1970 & 0.0000 & 0.0000 & 291.4982 & 0.0000 & 0.0000 & 0.0000 & 0.0000 & 0.0000 & 0.0000 \\
\hline P2O5 & 0.0140 & 2.2910 & 0.0321 & 0.0353 & 141.9370 & 0.0002 & 0.0160 & -53.6200 & -0.0086 & 0.0000 & -0.1212 \\
\hline$N+203$ & & 1.1660 & 0.0000 & 0.0000 & 336.4782 & 0.0000 & 0.0000 & -28.3200 & 0.0000 & 0.0000 & 0.0000 \\
\hline $\mathrm{LeO}$ & & 1.1728 & 0.0000 & 0.0000 & 325.8100 & 0.0000 & 0.0000 & -31.1400 & 0.0000 & 0.0000 & 0.0000 \\
\hline $\mathrm{Y2O3}$ & & 1.2699 & 0.0000 & 0.0000 & 225.8082 & 0.0000 & 0.0000 & -12.9500 & 0.0000 & 0.0000 & 0.0000 \\
\hline ED & 0.0050 & 1.1165 & 0.0056 & 0.0062 & 153.3394 & 0.0000 & 0.0026 & -30.5700 & -0.0008 & 0.0001 & -0.0111 \\
\hline$P \infty$ & & 1.0772 & 0.0000 & 0.0000 & 223.1900 & 0.0000 & 0.0000 & .8 .8100 & 0.0000 & 0.0000 & 0.0000 \\
\hline 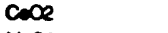 & & 1.2284 & 0.0000 & 0.0000 & 172.1200 & 0.0000 & 0.0000 & $\$ 1.3450$ & 0.0000 & 0.0000 & 0.0000 \\
\hline$m \infty$ & & 1.5003 & 0.0000 & 0.0000 & 143.9382 & 0.0000 & 0.0000 & -21.7500 & 0.0000 & 0.0000 & 0.0000 \\
\hline $2 \pi$ & 0.101 & 1.2447 & 0.1257 & 0.1385 & 81.3800 & 0.0015 & 0.1093 & -2.4000 & -0.0026 & 0.0022 & -0.0371 \\
\hline a) & & 1.2518 & 0.0000 & 0.0000 & 79.5454 & 0.0000 & 0.0000 & 1.7900 & 0.0000 & 0.0000 & 0.0000 \\
\hline $\mathrm{F} \bullet 2+1 \mathrm{~F} \bullet 3+$ & 0 & & & & & & & & & & \\
\hline Cl & 0.2272 & & 0.2272 & 0.2272 & & & & & & & \\
\hline $\mathbf{F}$ & $N / A$ & & N/A & $N / A$ & & & & & & & \\
\hline so4 & 0.1485 & & 0.1485 & 0.1485 & & & & & & & \\
\hline \multirow[t]{2}{*}{ OXDE SUMS } & 48.0820 & & 90.7513 & 100.0000 & & 1.4131 & 100.0000 & & -5.8329 & 0.4652 & -82.4268 \\
\hline & & & & & NORMALIZED & & & \multirow{3}{*}{$\begin{array}{c}S U M+p H S i \\
S U M+p H B \\
p H=\end{array}$} & -5.8329 & & \\
\hline \multicolumn{2}{|c|}{ MOLAR BALANCE (M) } & & MOLAR SUMS & MOA E\% & MOLE * & & & & -5.8329 & & \\
\hline $\mathrm{K}_{2} \mathrm{SO}_{4}$ & 0.2694 & & SALTS & 0.7469 & 0.6663 & & & & & & \\
\hline $\mathrm{KCl}$ & 0.4775 & & BORATES & 20.4065 & 18.2042 & & & & & & \\
\hline KBF4 & & & CHPOMATES & 0.1931 & 0.1723 & & & & & & \\
\hline NaBOe & 20.4065 & & OXIDES & 90.7513 & 80.9573 & & & & & & \\
\hline \multirow[t]{2}{*}{$\mathrm{Na2} \mathrm{CrO}_{4}$} & 0.1931 & & & & & & & & & & \\
\hline & & & TOTAL & 112.0977 & 100.0000 & & & & & & \\
\hline$K$ excess $(M)$ & 0.00 & & & & & & & & & & \\
\hline Ma excess (M) & 0.08 & & & & & & & & & & \\
\hline 804 oxcess (M & 0.00 & & & & & & & & & & \\
\hline $\begin{array}{l}\text { Cl excoss } \\
\text { F excosss }\end{array}$ & 0.00 & & & & & & & & & & \\
\hline E excess & 0.00 & & & & & & & & & & \\
\hline ALKSIO2 = & & 0.442 & & TEST $=$ & & & & & & & \\
\hline $\mathrm{ALK}+\mathrm{B2O} / \mathrm{SiO}$ & & 0.648 & & TIME: & & & & & & & \\
\hline $\mathrm{B2O3/S102}=$ & & 0.206 & & & & & & & & & \\
\hline vscosity QTE & $\mathrm{AP}\left({ }^{\circ} \mathrm{C}\right)$ & 28.736 & & SAIV $=$ & & & & & & & \\
\hline LQUDOUS RATIO & & 0.077 & & & & & & & & & \\
\hline UOUIDUS $\left({ }^{\circ} \mathrm{C}\right)=$ & Uscosty & 945.6550561 & 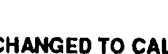 & 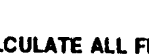 & 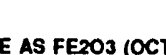 & 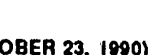 & & & & & \\
\hline
\end{tabular}

(SPREAOSHEET REVISION 5.0, OCTOBER 23, 1990) 
GLASS ID $=2 / 3+9$ CENTER - A (ADS \#200038850)

\begin{tabular}{|c|c|c|c|c|c|c|c|c|c|c|c|}
\hline & $\begin{array}{l}\text { ELEMENT } \\
\text { WT\% }\end{array}$ & $\begin{array}{l}\text { GRAV } \\
\text { FACTOR }\end{array}$ & $\begin{array}{l}\text { OXIDE } \\
W T \%\end{array}$ & $\begin{array}{c}\text { NOPMOXIDE } \\
\text { WT\% }\end{array}$ & N.W. & $\begin{array}{c}\text { MOLE } \\
\text { FAACTON }\end{array}$ & $\begin{array}{l}\text { NORMOXIDE } \\
\text { MOLE \% }\end{array}$ & $\begin{array}{l}\text { COMPONENT } \\
\text { FREE ENETGYY }\end{array}$ & $\begin{array}{l}\text { FREE ENERGY } \\
\text { HYDRATION }\end{array}$ & NBO & KCAL/KG \\
\hline $\mathrm{A} \mid 203$ & 1.6170 & 1.8895 & 3.0553 & 3.3476 & 101.9600 & 0.0300 & 2.1102 & 3.0400 & 0.0641 & .0 .0422 & 0.9110 \\
\hline$\infty$ & 0.6250 & 1.3992 & 0.8745 & 09581 & 56.0794 & 0.0156 & 1.0981 & -16.1160 & -0.1770 & 0.0220 & -2.5131 \\
\hline$F \bullet 203$ & 6.8750 & 1.4297 & 9.8292 & 10.7693 & 159.6922 & 0.0616 & 4.3344 & 15.5000 & 0.6718 & -0.0867 & 9.5404 \\
\hline$F \infty$ & N/A & 1.2865 & 0.0000 & 0.0000 & 71.8464 & 0.0000 & 0.0000 & -14.6090 & 0.0000 & 0.0000 & 0.0000 \\
\hline Mo & 0.4260 & 1.6583 & 0.7064 & 0.7740 & 40.3111 & 0.0175 & 1.2341 & $\cdot 13.8880$ & -0.1714 & 0.0247 & -2.4338 \\
\hline MHO & 1.5000 & 1.2912 & 1.9368 & 2.1221 & 70.9374 & 0.0273 & 1.9227 & -14.8710 & -0.2859 & 0.0385 & -4.0602 \\
\hline $\mathrm{NazO}$ & 9.0120 & 1.3480 & 12.1482 & 13.3101 & 61.9790 & 0.1960 & 13.8025 & -28.3150 & -3.9772 & 0.2761 & -56.4788 \\
\hline Li2O & 1.8550 & 2.1525 & 3.9929 & 4.3748 & 29.8774 & 0.1336 & 9.4110 & -22.7400 & -2.1401 & 0.1882 & -30.3903 \\
\hline $\mathrm{NOO}$ & 0.6430 & 1.2726 & 0.8183 & 0.8965 & 74.7094 & 0.0110 & 0.7713 & .14 .3470 & -0.1107 & 0.0154 & -1.5714 \\
\hline SiO2 & 21.1790 & 2.1393 & 45.3082 & 49.6418 & 60.0848 & 0.7541 & 53.1013 & 5.5900 & 1.3430 & 0.0000 & 19.0715 \\
\hline $\mathrm{Cr} 2 \mathrm{O} 3$ & 0.0690 & 1.4616 & 0.1009 & 0.1105 & 151.9974 & 0.0007 & 0.0467 & 37.3600 & 0.0175 & -0.0008 & 0.2479 \\
\hline 8203 & 3.4390 & 3.2201 & 11.0739 & 12.1331 & 69.6204 & 0.1591 & 11.2010 & -9.9300 & -1.1123 & 0.0000 & -15.7948 \\
\hline Uoe & & 1.1344 & 0.0000 & 0.0000 & 270.0388 & 0.0000 & 0.0000 & -6.8000 & 0.0000 & 0.0000 & 0.0000 \\
\hline Thor & & 1.1379 & 0.0000 & 0.0000 & 264.0400 & 0.0000 & 0.0000 & -2.5330 & 0.0000 & 0.0000 & 0.0000 \\
\hline sro & 0.0320 & 1.1826 & 0.0378 & 0.0415 & 103.6194 & 0.0004 & 0.0257 & -24.4000 & -0.0063 & 0.0005 & .0 .0891 \\
\hline $\mathrm{ZrO2}$ & 0.4940 & 1.3508 & 0.6673 & 0.7311 & 123.2188 & 0.0054 & 0.3814 & 45.1000 & 0.1720 & 0.0153 & 2.4424 \\
\hline $\mathrm{THO} 2$ & 0.0770 & 1.6680 & 0.1284 & 0.1407 & 79.8988 & 0.0016 & 0.1132 & 15.9900 & 0.0181 & 0.0045 & 0.2570 \\
\hline $\mathrm{K} 20$ & 0.3580 & 1.2046 & 0.4312 & 0.4725 & 94.2034 & 0.0046 & 0.3224 & -41.7350 & -0.1345 & 0.0064 & -1.8106 \\
\hline $\cos 0$ & & 1.0602 & 0.0000 & 0.0000 & 281.8094 & 0.0000 & 0.0000 & .46 .8200 & 0.0000 & 0.0000 & 0.0000 \\
\hline 55203 & & 1.1970 & 0.0000 & 0.0000 & 291.4982 & 0.0000 & 0.0000 & 0.0000 & 0.0000 & 0.0000 & 0.0000 \\
\hline P205 & 0.0150 & 2.2910 & 0.0344 & 0.0377 & 141.9370 & 0.0002 & 0.0170 & .53 .6200 & -0.0091 & 0.0000 & -0.1298 \\
\hline $\mathrm{N}+2 \mathrm{O} 3$ & & 1.1660 & 0.0000 & 0.0000 & 336.4782 & 0.0000 & 0.0000 & -28.3200 & 0.0000 & 0.0000 & 0.0000 \\
\hline $\mathrm{La2O3}$ & & 1.1728 & 0.0000 & 0.0000 & 325.8100 & 0.0000 & 0.0000 & -31.1400 & 0.0000 & 0.0000 & 0.0000 \\
\hline $\mathrm{rzO3}$ & & 1.2699 & 0.0000 & 0.0000 & 225.8082 & 0.0000 & 0.0000 & -12.9500 & 0.0000 & 0.0000 & 0.0000 \\
\hline $\mathrm{BaO}$ & 0.0050 & 1.1165 & 0.0056 & 0.0061 & 153.3394 & 0.0000 & 0.0026 & $\cdot 30.5700$ & -0.0008 & 0.0001 & -0.0111 \\
\hline$P \infty 0$ & & 1.0772 & 0.0000 & 0.0000 & 223.1900 & 0.0000 & 0.0000 & -8.8100 & 0.0000 & 0.0000 & 0.0000 \\
\hline code & & 1.2284 & 0.0000 & 0.0000 & 172.1200 & 0.0000 & 0.0000 & 11.3450 & 0.0000 & 0.0000 & 0.0000 \\
\hline$M \infty O B$ & & 1.5003 & 0.0000 & 0.0000 & 143.9382 & 0.0000 & 0.0000 & -21.7500 & 0.0000 & 0.0000 & 0.0000 \\
\hline $\mathbf{Z n O}$ & 0.097 & 1.2447 & 0.1207 & 0.1323 & 81.3800 & 0.0015 & 0.1045 & -2.4000 & -0.0025 & 0.0021 & -0.0356 \\
\hline a & & 1.2518 & 0.0000 & 0.0000 & 79.5454 & 0.0000 & 0.0000 & 1.7900 & 0.0000 & 0.0000 & 0.0000 \\
\hline $\mathrm{F}_{\theta 2+} / \mathrm{F}_{\theta 3+}$ & 0 & & & & & & & & & & \\
\hline Cl & 0.3768 & & 0.3768 & 0.3768 & & & & & & & \\
\hline $\mathbf{F}$ & $N / A$ & & $N / A$ & N/A & & & & & & & \\
\hline 504 & 0.1098 & & 0.1098 & 0.1098 & & & & & & & \\
\hline \multirow[t]{2}{*}{ OXIDE SUMS } & 48.3180 & & 91.2701 & 100.0000 & & 1.4201 & 100.0000 & & .5 .8412 & 0.4639 & .82 .9485 \\
\hline & & & & & NORMALLEED & & & SUM + pH Si & -5.8412 & & \\
\hline \multicolumn{2}{|c|}{ NOLAR BALANCE (M) } & \multicolumn{2}{|r|}{ MOLAR SUMB } & MOLEX & MOLE $\%$ & & & $\begin{array}{c}\mathrm{SUM}+\mathrm{pHB} \\
\mathrm{pH}=\end{array}$ & -5.8412 & & \\
\hline $\mathrm{K}_{2} \mathrm{SO}_{4}$ & 0.1992 & & SALTS & 0.9910 & 0.8739 & & & & & & \\
\hline $\mathrm{KCl}$ & 0.7919 & & BOPATES & 20.9299 & 18.4557 & & & & & & \\
\hline KBF4 & & & CHAOMATES & 0.2149 & 0.1895 & & & & & & \\
\hline $\mathrm{NaBO} 2$ & 20.9299 & & OXIDES & 91.2701 & 80.4809 & & & & & & \\
\hline \multirow[t]{2}{*}{$\mathrm{Na} 2 \mathrm{CrO} 4$} & 0.2149 & & & & & & & & & & \\
\hline & & & TOTAL. & 113.4059 & 100.0000 & & & & & & \\
\hline
\end{tabular}

(SPREADSHEET REVISION 5.0, OCTOBER 23, 1990)

0.0000

4.0602

8.4788

$-30.3903$

19.0715

0.2479

.7948

0.0000

0.0891

.2570

0.1298

0.0000

.0111

0.0000

0.0356

\section{$K \operatorname{excess}(M)$}

$\begin{array}{ll}\text { Na excess (M) } & 0.07 \\ \text { SO4 excess (M) } & 0.00 \\ \text { CI excess } & 0.00 \\ F \text { excess } & \\ \text { B excoss } & 0.00\end{array}$

B excoss

0.00

$\begin{array}{lcc}\text { ALKSIO2 }= & 0.443 & \text { TEST }= \\ \text { ALK+ B2O3/SIO2 } & 0.654 & \text { TIME= } \\ \text { B2O3/SIO2 }= & 0.211 & \\ \text { VISCOSITY OTEMP('C) } & 28.005 & \text { SA/V }= \\ \text { UOUDUS RATIO }= & 0.077 & \\ \text { UQUIDUS }\left({ }^{\circ} \mathrm{C}\right)= & 945.943831 & \end{array}$

TO 
GLASS ID $=2 / 3+9$ CENTER - B (ADS 200038851 )

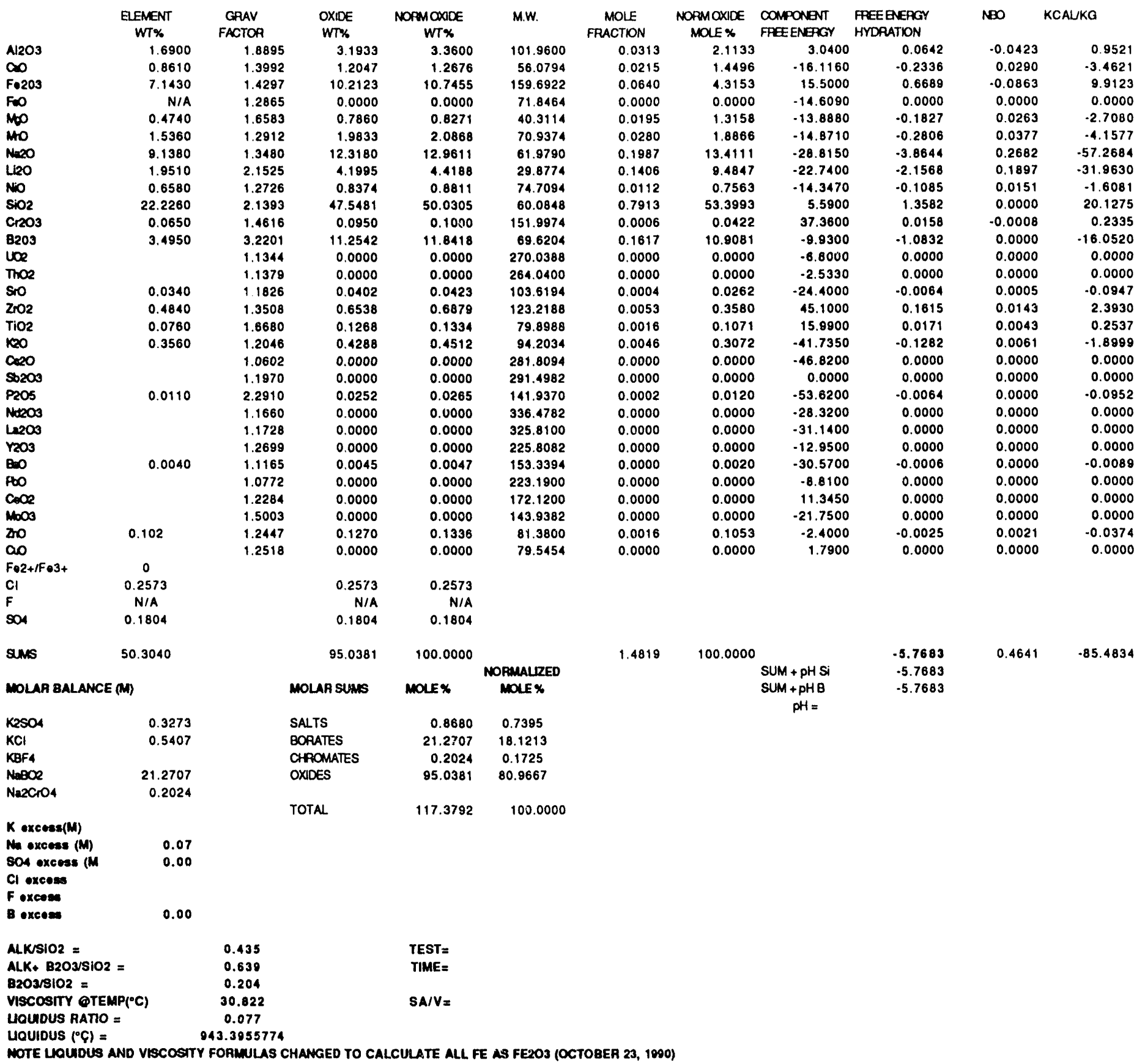

(SPREAOSHEET REVISION 5.0, OCTOBER 23, 1990) 
This page intentionally left blank. 
cc: $\quad$ L.M. Papouchado, 773-A

E. W. Holtzscheiter, 773-A

M.J. Plodinec, 773-A

W.T. Goldston, 704-S

J. T. Carter, 704-1T

C. T. Randall, 704-1T

L. F. Landon, 704-1T

N. E. Bibler, 773-A

C.M. Jantzen, 773-A

G. G. Wicks, 773-A

A. A. Ramsey, 704-15S

W. G. Ramsey, 773-A

M. K. Andrews, 773-62A

B. C. $\mathrm{Ha}, 773-\mathrm{A}$

K. E. Mottell, 773-43A

R. F. Schumacher, 773-42A

D. C. Beam, 773-A

D. F. Bickford, 773-A

S. L. Marra, 704-15S

C. A. Cicero, 773-62A

D. M. Ferrara, 773-62A

J. R. Harbour, 773-43A

J. F. Sproull, 704-S

M.E. Smith, 704-1T

N.D. Hutson, 704-1T

D.H. Miller, 704-1T

A.S. Choi, 704-1T

L. O. Westphal, 704-S

SRL Records, 773-A 

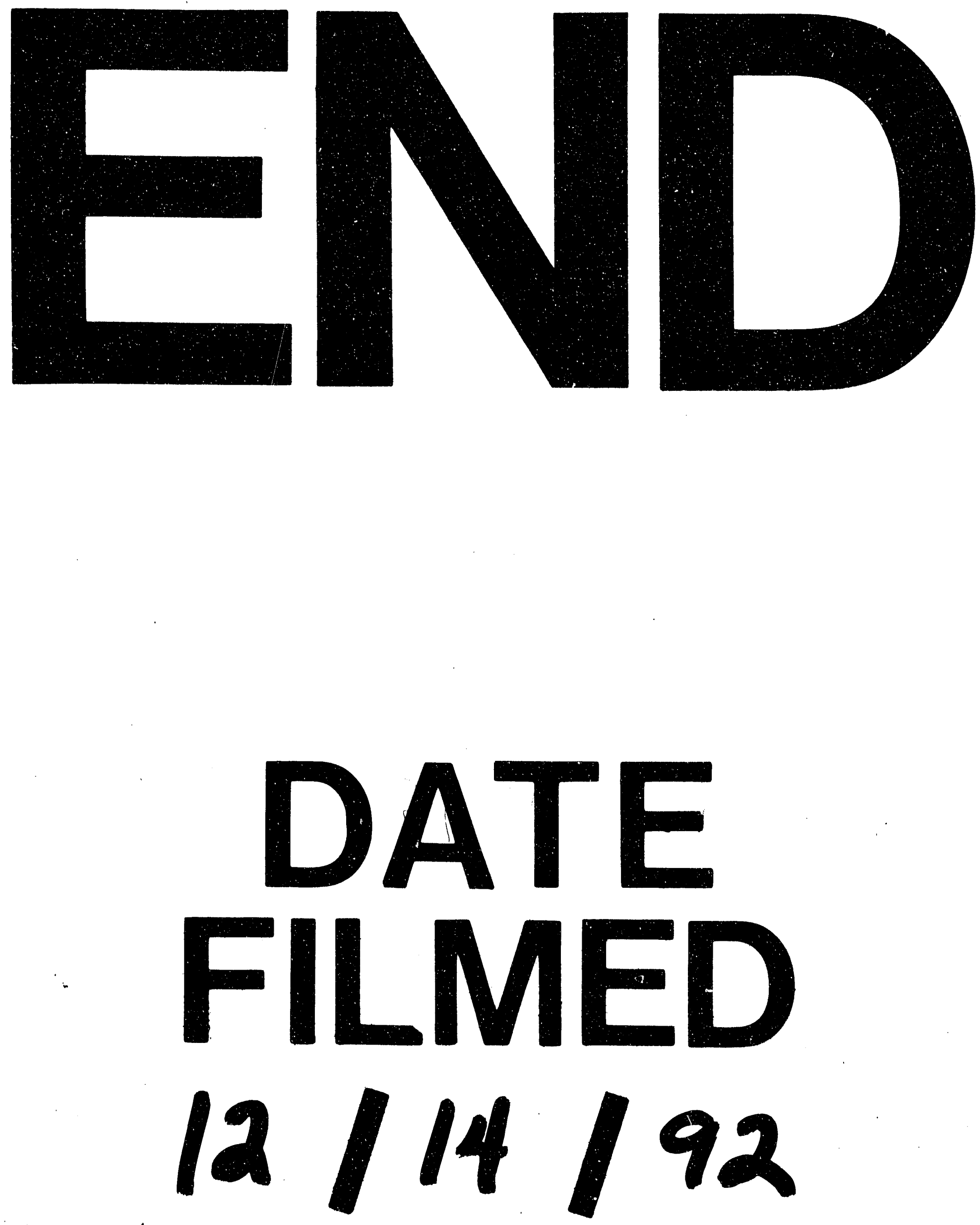